\title{
New insights in deep brain stimulation for Tourette syndrome
}

Citation for published version (APA):

Smeets, A. Y. J. M. (2018). New insights in deep brain stimulation for Tourette syndrome. [Doctoral Thesis, Maastricht University]. Datawyse / Universitaire Pers Maastricht. https://doi.org/10.26481/dis.20180601as

Document status and date:

Published: 01/01/2018

DOI:

10.26481/dis.20180601as

Document Version:

Publisher's PDF, also known as Version of record

\section{Please check the document version of this publication:}

- A submitted manuscript is the version of the article upon submission and before peer-review. There can be important differences between the submitted version and the official published version of record.

People interested in the research are advised to contact the author for the final version of the publication, or visit the DOI to the publisher's website.

- The final author version and the galley proof are versions of the publication after peer review.

- The final published version features the final layout of the paper including the volume, issue and page numbers.

Link to publication

\footnotetext{
General rights rights.

- You may freely distribute the URL identifying the publication in the public portal. please follow below link for the End User Agreement:

www.umlib.nl/taverne-license

Take down policy

If you believe that this document breaches copyright please contact us at:

repository@maastrichtuniversity.nl

providing details and we will investigate your claim.
}

Copyright and moral rights for the publications made accessible in the public portal are retained by the authors and/or other copyright owners and it is a condition of accessing publications that users recognise and abide by the legal requirements associated with these

- Users may download and print one copy of any publication from the public portal for the purpose of private study or research.

- You may not further distribute the material or use it for any profit-making activity or commercial gain

If the publication is distributed under the terms of Article $25 \mathrm{fa}$ of the Dutch Copyright Act, indicated by the "Taverne" license above, 


\section{New insights in Deep Brain Stimulation for Tourette Syndrome}

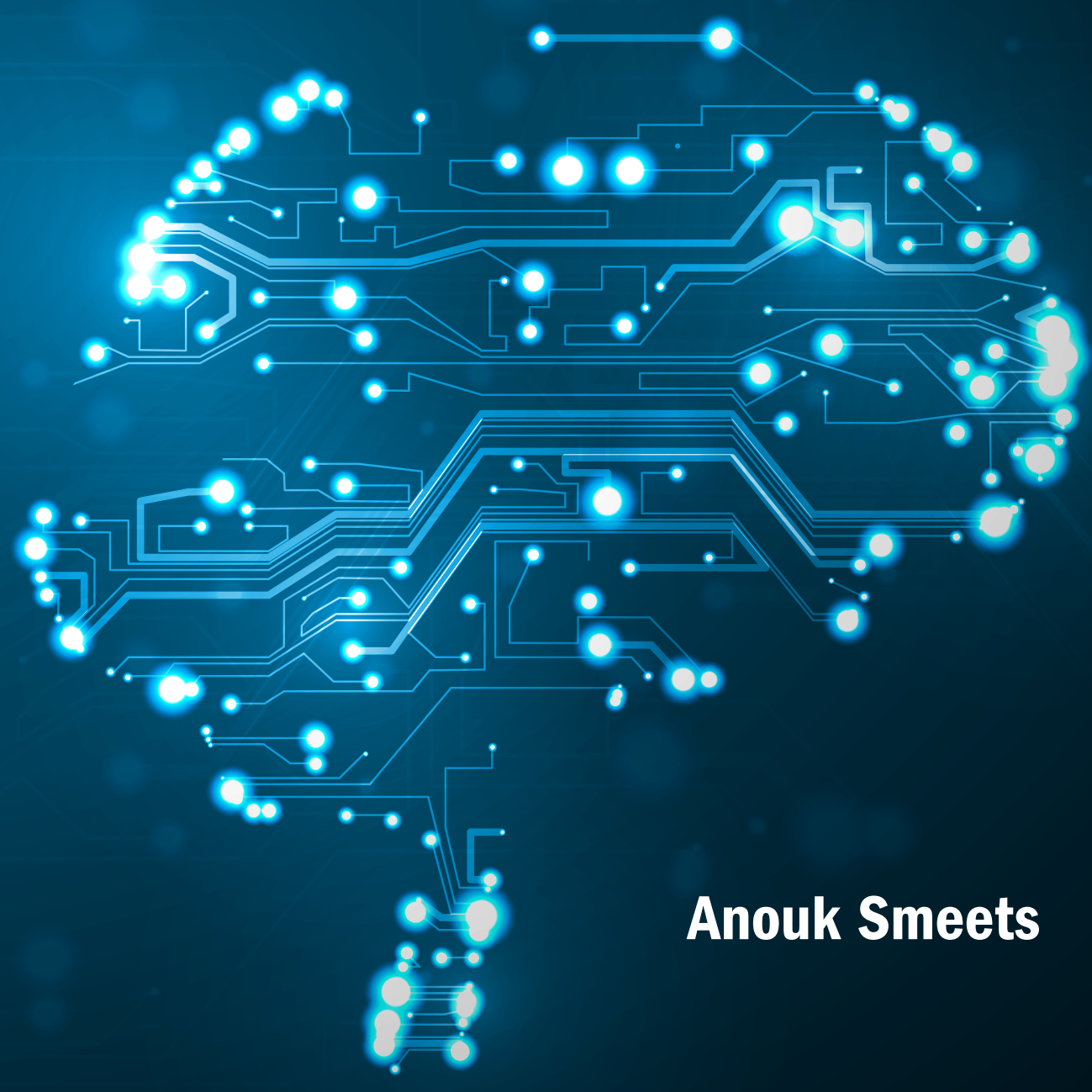


ISBN 9789461598196

Lay-out: Datawyse | Universitaire Pers Maastricht Cover design: Datawyse | Universitaire Pers Maastricht Production: Datawyse | Universitaire Pers Maastricht

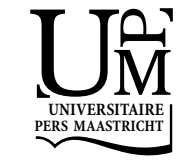

(C) Anouk Y.J.M Smeets, Maastricht, the Netherlands, 2018.

All rights reserved. No part of this thesis may be reproduced, stored in a retrieval system, or transmitted, in any form by any means, electronically, photocopying, or otherwise, without the permission of the author. The copyright of the articles that have been published has been transferred to the respective journals. This thesis was not financially supported 


\title{
New insights in Deep Brain Stimulation
}

\section{for Tourette Syndrome}

\author{
PROEFSCHRIFT
}

Ter verkrijging van de graad van doctor aan de Universiteit Maastricht, op gezag van de Rector Magnificus,

Prof. dr. Rianne M. Letschert, volgens het besluit van het College van Decanen, in het openbaar te verdedigen op vrijdag 1 juni 2018 om 12.00 uur

door

Anouk Yvonne Jacqueline Maria Smeets 
Promotor

Prof. Dr. Y Temel

\section{Copromotores}

Dr. L Ackermans

Dr. A.A Duits

Dr. A.F.G Leentjens

\section{Beoordelingscommissie}

Prof. Dr. B.P.F Rutten (voorzitter)

Dr. M Kuijf

Prof. Dr. W.H Mess

Prof. Dr. B Nuttin (Katholieke Universiteit Leuven)

Dr. S Tan (Universitätsklinikum Aachen) 
Voor mijn lieve opa 



\section{Contents}

Chapter 1 Introduction

A.Y.J.M Smeets, A.F.G Leentjens, L. Ackermans

Adjusted version in press as book chapter for: "Deep brain stimulation:

an interdisciplinary approach"

Chapter 2 The role of the basal ganglia in Tourette Syndrome

A.Y.J.M Smeets, A.F.G Leentjens, A.A Duits, Y Temel, L Ackermans

In preparation

Chapter 3 Thalamic Deep Brain Stimulation for refractory Tourette Syndrome:

clinical evidence for increasing disbalance of therapeutic effects and side effects at long-term follow-up

A.Y.J.M Smeets, A.A Duits, A.F.G Leentjens, K Schruers, V van Kranen-

Mastenbroek, $V$ Visser Vandewalle, Y Temel, L Ackermans

Neuromodulation 2017

Chapter 4 Deep Brain Stimulation of the internal globus pallidus in refractory

Tourette Syndrome

51

A.Y.J.M Smeets, A.A Duits, B.R Plantinga, A.F.G Leentjens, M Oosterloo, $\checkmark$ Visser-Vandewalle, Y Temel, L Ackermans

Clinical Neurology and Neurosurgery 2016

Chapter 5 Tonic and phasic changes in pallidal activity in Tourette Syndrome $M$ Israelashvili, A.Y.J.M Smeets, M Bronfeld, D.H Zeef, A.F.G Leentjens, $\checkmark$ van Kranen-Mastenbroek, M.L.F Janssen, Y Temel, L Ackermans, I Bar-Gad

Movement disorders 2017

Chapter 6 Modified cement-based fixation of the Deep Brain Stimulation

electrode

A.Y.J.M Smeets, L Ackermans, M Oosterloo, M.L Kuijf,

J.J van Overbeeke, V Visser-Vandewalle, Y Temel

Stereotactic and functional neurosurgery 2015 
Chapter 7 Ethics of Deep Brain Stimulation in adolescent patients with refractory Tourette Syndrome: a systematic review and two case discussions

A.Y.J.M Smeets, A.A Duits, C Verdellen, $G$ de Wert, D Horstkötter, Y Temel, L Ackermans, A. F. G Leentjens

Neuroethics 2018

Chapter 8 Discussion

Summary

Samenvatting

Valorisation 135

Dankwoord 139

Curriculum Vitae

145

List of publications

149 


\section{Chapter 1 Introduction}





\section{TOURETTE SYNDROME}

\section{Clinical picture}

George Gilles de la Tourette first described Tourette Syndrome (TS) in 1885 as a clinical triad of multiple tics, coprolalia and echolalia ${ }^{1}$. However, a publication dated 1873 by Armand Trousseau included a detailed account of what is now known as TS ${ }^{2}$. TS is a childhood-onset complex neuropsychiatric disorder characterized by tics ${ }^{3-5}$. According to the criteria of the fifth edition of the Diagnostic and Statistical Manual of mental disorders (DSM-5) ${ }^{6}$, multiple motor and one or more vocal tics must be present for more than one year and the onset of tics needs to be before the age of 18 . The DSM-5 criteria are listed in Table 1.

Table 1: DSM-5 criteria for Tourette Syndrome.

For a diagnosis of Tourette Syndrome the patient should meet all of the following criteria:

\begin{tabular}{ll}
\hline Criterion & Description \\
\hline A & $\begin{array}{l}\text { Both multiple motor and one or more vocal tics have been present at some time during the } \\
\text { illness, although not necessarily concurrently }\end{array}$ \\
B & $\begin{array}{l}\text { Tics may wax and wane in frequency, but have persisted for more than one year since first tic } \\
\text { onset }\end{array}$ \\
C & Onset is before age of 18 years \\
D & The disturbance is not attributable to the physiological effects of a substance (e.g. cocaine) or \\
& another medical condition (e.g. Huntington's disease, post viral encephalitis)
\end{tabular}

Tics are defined as sudden, rapid, recurrent, non-rhythmic muscle contractions that produce stereotyped movements (motor tics) or sounds (vocal tics). Tics may be abrupt in onset, fast and brief (clonic tics) or slow and sustained (dystonic or tonic tics) ${ }^{7,8}$. Tics in TS typically begin as simple motor tics (e.g. eye blinking, facial grimaces) around the age of 4-6 years, followed by simple vocal tics (e.g. throat clearing, sniffing) a year or two later. After onset of tics there is often a progressive pattern of tic worsening, and tics become more complex ${ }^{3}$. Coprolalia, one of the most debilitating symptoms, occurs in about $14-20 \%$ of patients ${ }^{9}$. Many patients describe premonitory sensations, the building up of tension just before a tic, and these sensations are often more distressful than the tics themselves. Once the tic has occurred, there is a brief experience of relief 7,8. Most patients can suppress tics for a short while and describe a rebound intensification of tics afterwards. In spite of the fact that many patients describe such a rebound, this phenomenon has not been confirmed in research ${ }^{10,11}$. The severity of tics can also be influenced by circumstances, they may increase with stress, anxiety and fatigue, while focused activities and alcohol use may reduce their intensity ${ }^{7,8}$. 


\section{Course of the disease}

The prevalence of TS in childhood (defined here as age $<18$ years) is estimated at 0.3 to $0.9 \%$ with males being affected two to four times as frequent as women ${ }^{9}$. On average, tics start around the age of 4-6 years, reach their worst-ever period early in the second decade of life, around 8-12 years of age, to be followed by a steady decline in severity ${ }^{3}$. In the natural course of TS, tics occur many times a day in bouts and wax and wane in severity over time. Overall, TS has a favourable prognosis. About one third of children with TS will experience a significant tic decrease during adolescence and remain with only mild tics that do not require clinical attention, whereas another third of children is completely tic-free ${ }^{3}$. Yet, a small proportion of patients does not experience this remission, and in this group some individuals not only experience tic worsening during adolescence but they develop the most severe and debilitating forms of TS. In $5 \%$ of patient's TS has a serious and even life-threatening nature, called malignant TS. It is characterized by tic-related injuries, Self-Injurious Behaviour (SIB), uncontrollable violence, and suicidal attempts ${ }^{3,9,12}$.

\section{Comorbidities}

TS is accompanied by comorbid disorders in $50-90 \%$ of patients causing a heterogeneous phenotype of the disease. In some patients, these comorbid disorders cause even more impairment than the tics themselves. Attention Deficit and Hyperactivity Disorder (ADHD) is present in about 50\% of patients and ObsessiveCompulsive behaviour (OCb) in 50-75\% ${ }^{3,4,13}$. Obsessions may involve violent, aggressive, sexual and symmetrical themes. Compulsive behaviour is often related to checking, counting, forceful touching and self-damage. More than one-third of patients with TS exhibits SIB, most frequently head banging. Many TS patients experience developmental disorders, learning disabilities, sleeping disorders, anxiety disorders and depression ${ }^{4,13}$.

\section{Pathophysiology}

The pathophysiology of TS is still largely unknown. In the past few years, a lot of research has been directed towards genetic and auto-immune disorders ${ }^{14}$. Functional and structural studies show abnormalities in several cortical and subcortical areas during tics, among which the basal ganglia (BG) ${ }^{14-18}$. The BG modulate behaviour through a mechanism that involves changing cortical excitability through different BG circuits. The direct circuit, that extends from the striatum to the internal globus pallidus (GPi) and the substantia nigra pars reticulata (SNr), excites the cortex through the disinhibition of the thalamus. The indirect pathway, extending from the striatum to the external globus pallidus (GPe) and then to the subthalamic nucleus (STN), inhibits the thalamic projections. The hyperdirect pathway bypasses the striatum and sends cortical 
signals directly to the STN and then to the GPi/SNr. In general, the direct pathway activates desired programs while the hyperdirect and indirect pathways suppress unwanted competing programs ${ }^{14}$. It has been hypothesized that in TS due to a dopaminergic dysfunction, focal aberrations in the striatum cause excessive inhibition of the GPi/SNr in the direct pathway, thereby causing an involuntary motor command to be executed in the cortex due to excessive disinhibition ${ }^{19}$. The cortico-basal-gangliathalamocortical (CBGT) projections can be divided into five important functional circuits, named for their cortical areas of origin and action, which travel through different parts of the BG nuclei: the motor, the associative, the limbic, the oculomotor and the orbitofrontal circuit. Chapter 2 will be dedicated to the functional anatomy of the BG and the relationship between BG dysfunction and the pathophysiology of TS.

\section{TREATMENT OF TOURETTE SYNDROME}

There is no cure for TS. Therefore, treatment aims to diminish or alleviate tics and other comorbid symptoms. The critical first step in making treatment decisions is to select the most appropriate target symptoms, the ones causing the most problems in a patient's daily functioning ${ }^{20}$. In one patient it may be the tics themselves, in another it may be comorbid ADHD or OCb and in another it may be a combination of target symptoms.

\section{Psychosocial or behavioural treatment}

For many patients with mild symptoms, psychosocial or behavioural interventions may be sufficient to bring symptoms to a tolerable level of severity ${ }^{5,20}$. Psychosocial interventions are for example psychoeducation which has the aim to improve the tolerance for symptoms and to support stress reduction ${ }^{21}$. Behavioural approaches are habit reversal therapy, relaxation training, contingency management, exposure and response prevention, self-monitoring, massed practice, bio(neuro)feedback, and cognitive behavioural treatment ${ }^{20,21}$. In habit reversal therapy, patients are trained to recognize premonitory urges and respond to these with a voluntary competing or incompatible response, such as contraction of a 'tic-opposing' muscle or relaxation of the 'tic-muscle'. Habit reversal therapy may also compromise relaxation training and contingency management. Relaxation techniques are focused on muscle relaxation in general and in response to premonitory urges. Contingency management consists of the manipulation of environmental contingencies, so that tic-free intervals are positively reinforced and tic behaviours are ignored. Exposure and response prevention consists of exposure to the premonitory sensory urges during prolonged tic suppression and stimulating habituation. 


\section{Pharmacological treatment}

Pharmacological treatment might be an option if psychosocial or behavioural interventions are not sufficient, for example if a patient experiences: tics that cause subjective discomfort (e.g. pain or injury), tics that cause sustained social problems (e.g. social insolation or bullying), tics that cause emotional problems (e.g. reactive depressive symptoms), or tics that cause functional interference (e.g. impairment of academic achievements) ${ }^{22}$. It is important to realize that it is still unknown what effect pharmacological treatment has on the natural long-term course of TS and how it might influence the natural course of brain development ${ }^{22}$. A large number of pharmacological interventions have shown effect in reducing tics in TS patients including typical and atypical antipsychotics, benzamides, noradrenergic agents, tetrabenazine and tetrahydrocannabinol ${ }^{4,8,22}$. Most widely used drugs are typical antipsychotics (pimozide, haloperidol, sulpiride, tiapride) that block postsynaptic dopamine-D2 receptors. In adult TS patients these are considered the first choice. Benzamides, such as tiapride and sulpiride, also work via the dopaminergic system. Alternatives are atypical antipsychotics (risperidone, olanzapine, quetiapine, ziprasidone) that have more serotonergic and noradrenergic effects. Another noradrenergic agent used in the treatment of tics is clonidine, which is often used as the first choice in children. Mild side effects like sedation and irritability are reported for all these types of drugs. But more severe side effects like extrapyramidal symptoms (e.g. tardive dyskinesia or parkinsonism), weight gain, impaired glucose tolerance and depression are also seen with these antipsychotics. The extrapyramidal side effects are the most pronounced for the typical antipsychotics ${ }^{22}$. Some evidence also exists for tetrabenazine and tetrahydrocannabinol. If tics are limited to one muscle or one muscle group, botulinum toxin injections can help. Selective serotonin reuptake inhibitors and clomipramine are recommended for the treatment of comorbid OCb and depression. Psychostimulants, such as methylphenidate, are used for the treatment of comorbid ADHD $^{4,8,22}$.

\section{Deep Brain Stimulation}

Despite psychosocial and behavioural therapy and pharmacological treatment, a small sub-group of patients fails to respond to treatment or experiences intolerable side effects. They continue to experience significant symptom burden throughout adolescence and adult life ${ }^{21,22}$. For those patients, Deep Brain Stimulation (DBS) can be considered. Guidelines for indication have been proposed by the Tourette Syndrome Association (TSA) ${ }^{23}$ (see Table 2). 
Table 2: Criteria for indicating Deep Brain Stimulation in Tourette Syndrome patients.

\begin{tabular}{|c|c|c|}
\hline & Criterion & \\
\hline Diagnosis & $\begin{array}{l}\text { A confirmed diagnosis of Tourette Syndrome according to the } \\
\text { DSM- } 5 \text { criteria }\end{array}$ & \\
\hline Age & $>18$ years & \\
\hline Severity & YGTSS >35/50 & \\
\hline $\begin{array}{l}\text { Neuropsychiatric } \\
\text { comorbidity }\end{array}$ & $\begin{array}{l}\text { - Tics must be the predominant complaint. If neuropsychiatric } \\
\text { comorbidity is present, this should not be predominant } \\
\text { - No suicidal ideation or attempted suicide in the past six } \\
\text { months } \\
\text { - Comorbid neuropsychiatric disorders must have been stable } \\
\text { for at least six months } \\
\text { - Comorbid neuropsychiatric morbidity should be scored with } \\
\text { valid rating scales, so that these symptoms can be } \\
\text { monitored post-operatively }\end{array}$ & \\
\hline Previous treatmen & $\begin{array}{l}\text { - Treatment with at least three medications must have shown } \\
\text { no effects or could not be tolerated } \\
\text { - Behavioural treatment was not effective }\end{array}$ & $\begin{array}{l}\text { - D2 receptor antagonists } \\
\text { - Atypical antipsychotics } \\
\text { - Alpha-adrenergic agonists } \\
\text { - Other } \\
\text { - Habit reversal therapy } \\
\text { - Exposure and response } \\
\text { prevention }\end{array}$ \\
\hline $\begin{array}{l}\text { Psychosocial } \\
\text { factors }\end{array}$ & $\begin{array}{l}\text { - Adequate social support without any (sub)acute stressors } \\
\text { - Willingness to undergo psychological treatment post- } \\
\text { operatively if necessary } \\
\text { - Preparedness of caregiver to participate in post-operative } \\
\text { counselling }\end{array}$ & \\
\hline
\end{tabular}

DSM-5: fifth edition of the Diagnostic and Statistical Manual of mental disorders; YGTSS: Yale Global Tic Severity Scale; D2: dopamine 2.

Obviously, there are also contra-indications for DBS such as severe systematic diseases or structural abnormalities on the Magnetic Resonance Imaging (MRI) that interfere with optimal or safe electrode placement. The final decision for proceeding to DBS should be made by consensus within a multidisciplinary team in which the neurosurgeon, psychiatrist and preferably the psychologist participates.

Since 1955 attempts have been made to treat TS surgically. A total of 65 patients have been treated with ablative surgery that was targeted at various brain areas, among which the frontal cortex, the thalamus, the globus pallidus and the cerebellum ${ }^{24}$. The results of these interventions have not been well described and severe complications occurred such as hemiplegia and dystonia. Based on new developments within movement disorder surgery, DBS of the thalamus was first used as treatment for intractable TS in $1999^{25}$. The procedure requires the electrical stimulation of deep brain structures by implanted electrodes which receive high-frequency pulses from a subcutaneous implanted pulse generator. The main goal of DBS is to change neuronal firing patterns and to influence the passage of information through the targeted nuclei in the CBGT circuits and to improve symptoms of TS. This has the same effect on symptoms as a lesion, but in a reversible way. 
Evidence shows that DBS exerts its effect by causing functional ablation which reduces information flow through the stimulated nucleus ${ }^{26,27}$.

Since 1999, about 57 DBS studies in TS have been published worldwide, including approximately 156 patients from 23 centres in 13 countries ${ }^{28}$. A key factor to attaining optimal results from this surgery is target selection, a topic still under debate due to the complex clinical profile presented by TS patients. Nine separate brain areas have been targeted so far:

- The medial part of the thalamus:

1) The centromedian nucleus, substantia periventricularis, and nucleus ventrooralis internus (Cm-Spv-Voi) cross point of the thalamus ${ }^{25,29-44}$.

2) The centromedian nucleus-parafascicular complex (CM-Pf) of the thalamus ${ }^{45-50}$.

3) The dorsomedian nucleus-parafascicular complex (DM-Pf) of the thalamus ${ }^{51}$.

- The internal globus pallidus (GPi):

4) The posterolateral motor part (posterior GPi) ${ }^{52-63}$.

5) The anteromedial limbic part (anterior GPi) ${ }^{61,64-71 .}$

- 6) The external globus pallidus (GPe) ${ }^{72}$.

- 7) The nucleus accumbens (NA) ${ }^{73-76}$.

- 8) The anterior limb of the internal capsule (ALIC) ${ }^{73-75,77 .}$

- 9) The subthalamic nucleus (STN) ${ }^{78}$.

Moreover, studies targetting multiple brain areas separate or at the same time have been performed ${ }^{52,79-88}$. Interfering with DBS at the level of one of the main output or relay stations of the $B G$ seems to result in clinical benefits in refractory TS patients. Overall, DBS results in a significant short-term improvement of 53\% on the Yale Global Tic Severity Scale (YGTSS) on all targets (Table 3). The analysis of controlled studies shows a significant improvement in tics during ON stimulation compared to OFF stimulation. In general, the degree of tic improvement appeared to be most robust for the thalamic and the GPi targets ${ }^{28}$.

Table 3: Effects of Deep Brain Stimulation in Tourette Syndrome patients.

\begin{tabular}{lrlll}
\hline Target & N & Mean change (\%)* & $\geq 25 \%$ response (\%)* & $\geq 50 \%$ response (\%)* \\
\hline Thalamus & 78 & $53(26)$ & 82 & 52 \\
Posterior GPi & 20 & $57(35)$ & 78 & 61 \\
Anterior GPi & 44 & $52(55)$ & 77 & 66 \\
GPe/STN & 2 & - & - & - \\
NA/ALIC & 9 & $44(15)$ & 75 & 38 \\
Other & 3 & $45(27)$ & 67 & 67 \\
Total & 156 & $53(27)$ & 81 & 54 \\
\hline
\end{tabular}

* Mean change in score and percentage response on the Yale Global Tic Severity Scale (YGTSS).

\# Other: area of the GPi targeted was unclear $(n=2)$ and stimulation in both anterior and posterior GPi $(n=1)$. Abbreviations: N: number; GPi: internal globus pallidus; NA: nucleus accumbens; ALIC: anterior limb of the internal capsule; GPe: external globus pallidus; STN: subthalamic nucleus.

Table adapted from article by Baldermann et al $^{28}$. 


\section{DBS of the medial thalamus}

The first DBS implantation for TS in three patients targeted the $\mathrm{Cm}$-Spv-Voi cross point of the thalamus and resulted in a tic reduction of $72 \%, 83 \%$ and $90 \%$ on the YGTSS, respectively ${ }^{43}$. The choice for this target was based on the successful stereotactic lesioning in a case described by Hassler and Dieckmann ${ }^{89}$. The hypothesis was that stimulation of the $\mathrm{Cm}$ and Spv would supress excitatory feedback projection to motor and limbic parts of the striatum, decrease tics and improve behavioural disorders. Stimulation of the Voi was supposed to include a projection to the facial part of the premotor cortex allowing modulation of orofacial tics. Since then, 78 patients with thalamic DBS have been reported in 22 studies ${ }^{28}$. The mean percentage improvement on the YGTSS was $53 \%$, with $52 \%$ of patients reporting at least $50 \%$ improvement at the last reported follow-up (Table 3 ). This follow-up varied widely and only $20 \%$ of included patients had a follow-up of more than one year. Reported side effects include loss of libido, lethargy, and weight loss. Cases of psychosis, depression and hypomania have been reported and these may be reversed by adjusting stimulation parameters ${ }^{90}$.

\section{DBS of the globus pallidus}

The globus pallidus consists of an internal and external part (GPi and GPe), and most studies have targeted the GPi. Only one case history reports on stimulation of the GPe in TS with a 70\% improvement on the YGTSS after six months ${ }^{72}$. The GPi consists of a posterolateral motor part (posterior GPi) and an anteromedial limbic part (anterior GPi). Both have been targeted in the DBS treatment of TS. Some studies do not report which part of the GPi is targeted. DBS of the posterior GPi was first done in 2002 with good results ${ }^{52}$ and since then 20 patients have been described in the literature ${ }^{28}$. A mean improvement on the YGTSS of $57 \%$ has been reported, with $61 \%$ of patients reporting at least 50\% improvement at the last reported follow-up (Table 3). By now, more patients have received DBS of the anterior $\mathrm{GPi}^{28}$. These patients show a mean improvement of $52 \%$ on the YGTSS with $66 \%$ of patients reporting at least $50 \%$ improvement (Table 3 ). Known side effects of pallidal DBS are anxiety, agitation, weight loss, nausea, vertigo and paraesthesia's of which most appeared to be transient ${ }^{90}$.

\section{DBS of the nucleus accumbens and the anterior limb of the internal capsule}

Only 9 patients have been reported that received DBS of the NA/ALIC with a mean improvement of $44 \%$ on the YGTSS, and 38\% reporting more than $50 \%$ improvement ${ }^{28}$ (Table 3). Although the evidence is weak, stimulation of this target seems to be less effective. Potential side effects of DBS of the NA include flushing, anxiety, sweating, hypomania, agitation, and psychosis. Potential side effects of ALIC DBS are euphoria, anxiety, panic, and fear ${ }^{90}$. 
DBS of the subthalamic nucleus

A single case history of a 38-year-old man with Parkinson's disease (PD) who also suffered from tics has been described. After DBS of the STN for his PD, not only parkinsonian motor symptoms improved, but the tic frequency also diminished by $97 \%$ at one year ${ }^{78}$. 


\section{AIM OF THIS THESIS}

The general aim of this thesis is to continue the development of DBS for refractory TS patients by further exploring the effects of surgery in our own centre, including the relationships with the underlying pathophysiology, and both surgical and ethical issues. Open label series and small double-blind trials report positive effects on tics in TS patients after DBS of several brain targets, including the medial thalamus and the anterior GPi. However, DBS for TS is still not approved by the European Commission (EC) and the Food and Drug Administration (FDA) because of the lack of evidence due to the difficulty of performing high quality research in a randomized controlled fashion, coupled with the variable effect of surgery, the large number of targets, and the overall small numbers of TS patients with DBS worldwide. The main focus of this thesis is to continue the follow-up of operated TS patients and TS patients with an indication for DBS, including both thalamic and anterior GPi DBS. To goal is to provide new insights about the effects of thalamic and pallidal DBS for refractory TS patients.

Chapter 2 provides an overview of the functional anatomy of the BG and the connected CBGT pathways and explores the relation between BG dysfunction and the pathophysiology of TS. We explain how targeting different brain areas within the BG might influence these pathways in order to improve symptoms of TS. In chapter 3 we report on the long-term outcome of seven refractory TS patients who were participants of a double blinded randomized controlled trial, treated with DBS of the medial thalamus between 2001 and 2008. Because of our own results with thalamic DBS, showing an increasing disbalance of therapeutic effects and side effects at long-term follow-up, and the positive results of other groups targeting the anterior GPi, we changed our target. Chapter 4 describes the results of DBS of the anterior GPi in five refractory TS patients. In chapter 5 we describe our electrophysiology recordings during DBS surgery targeting the anterior GPi in eight awake TS patients. We have directly observed neuronal activity in the limbic domains of the BG and this chapter focuses on linking the activity of GPi neurons in these domains with the underlying pathophysiology of TS. Every step of the DBS surgery is important, and as such chapter 6 describes a modified cement-based fixation of the DBS electrode to prevent unintended migration, and consequently displacement of the electrodes. Chapter 7 deals with the ethical issues associated with DBS of adolescent TS patients. We describe our experiences with the treatment of two adolescent TS patients and systematically review the scarce literature on the subject. Finally, we provide a general discussion in chapter 8 in which we address questions about the underlying pathophysiology of TS and we provide new insights about the effects of thalamic and pallidal DBS for refractory TS patients. 


\section{REFERENCES}

1. Kramer H, Daniels C. Pioneers of movement disorders: Georges Gilles de la Tourette. Journal of neural transmission. 2004;111(6):691-701.

2. Rickards H, Woolf I, Cavanna AE. "Trousseau's disease:" a description of the Gilles de la Tourette syndrome 12 years before 1885. Movement disorders. 2010;25(14):2285-2289.

3. Bloch $\mathrm{MH}$, Leckman JF. Clinical course of Tourette syndrome. Journal of psychosomatic research. 2009;67(6):497-501.

4. Robertson MM. Tourette syndrome, associated conditions and the complexities of treatment. Brain. 2000;123 Pt 3:425-462.

5. Robertson MM. A personal 35 year perspective on Gilles de la Tourette syndrome: assessment, investigations, and management. Lancet Psychiatry. 2015;2(1):88-104.

6. Association AP. Diagnostic and statistical manual of mental disorders (DSM-5). Washington 2013.

7. Jankovic J, Kurlan R. Tourette syndrome: evolving concepts. Movement disorders. 2011;26(6):1149-1156.

8. Leckman JF. Tourette's syndrome. Lancet. 2002;360(9345):1577-1586.

9. Robertson MM. The prevalence and epidemiology of Gilles de la Tourette syndrome. Part 1: the epidemiological and prevalence studies. Journal of psychosomatic research. 2008;65(5):461-472.

10. Muller-Vahl KR, Riemann L, Bokemeyer S. Tourette patients' misbelief of a tic rebound is due to overall difficulties in reliable tic rating. Journal of psychosomatic research. 2014;76(6):472-476.

11. Verdellen CW, Hoogduin CA, Keijsers GP. Tic suppression in the treatment of Tourette's syndrome with exposure therapy: the rebound phenomenon reconsidered. Movement disorders. 2007;22(11):16011606.

12. Cath DC, Hedderly T, Ludolph AG, et al. European clinical guidelines for Tourette syndrome and other tic disorders. Part I: assessment. European child \& adolescent psychiatry. 2011;20(4):155-171.

13. Leckman JF, Bloch MH, Scahill L, King RA. Tourette syndrome: the self under siege. Journal of child neurology. 2006;21(8):642-649.

14. Mink JW. Basal ganglia dysfunction in Tourette's syndrome: a new hypothesis. Pediatric neurology. 2001;25(3):190-198.

15. Albin RL, Mink JW. Recent advances in Tourette syndrome research. Trends in neurosciences. 2006;29(3):175-182.

16. Bloch MH, Leckman JF, Zhu H, Peterson BS. Caudate volumes in childhood predict symptom severity in adults with Tourette syndrome. Neurology. 2005;65(8):1253-1258.

17. Peterson BS, Thomas P, Kane MJ, et al. Basal Ganglia volumes in patients with Gilles de la Tourette syndrome. Archives of general psychiatry. 2003;60(4):415-424.

18. Rauch SL, Whalen PJ, Curran T, et al. Probing striato-thalamic function in obsessive-compulsive disorder and Tourette syndrome using neuroimaging methods. Advances in neurology. 2001;85:207-224.

19. Singer HS, Minzer K. Neurobiology of Tourette's syndrome: concepts of neuroanatomic localization and neurochemical abnormalities. Brain \& development. 2003;25 Suppl 1:S70-84.

20. Shprecher D, Kurlan R. The management of tics. Movement disorders. 2009;24(1):15-24.

21. Verdellen C, van de Griendt J, Hartmann A, Murphy T, Group EG. European clinical guidelines for Tourette syndrome and other tic disorders. Part III: behavioural and psychosocial interventions. European child \& adolescent psychiatry. 2011;20(4):197-207.

22. Roessner V, Plessen KJ, Rothenberger A, et al. European clinical guidelines for Tourette syndrome and other tic disorders. Part II: pharmacological treatment. European child \& adolescent psychiatry. 2011;20(4):173-196.

23. Schrock LE, Mink JW, Woods DW, et al. Tourette syndrome deep brain stimulation: A review and updated recommendations. Movement disorders. 2014.

24. Temel Y, Visser-Vandewalle V. Surgery in Tourette syndrome. Movement disorders. 2004;19(1):3-14.

25. Vandewalle V, van der Linden C, Groenewegen HJ, Caemaert J. Stereotactic treatment of Gilles de la Tourette syndrome by high frequency stimulation of thalamus. Lancet. 1999;353(9154):724. 
26. Israelashvili M, Loewenstern Y, Bar-Gad I. Abnormal neuronal activity in Tourette syndrome and its modulation using deep brain stimulation. Journal of neurophysiology. 2015;114(1):6-20.

27. Zhuang $P$, Hallett $M$, Zhang $X$, Li J, Zhang $Y$, Li Y. Neuronal activity in the globus pallidus internus in patients with tics. Journal of neurology, neurosurgery, and psychiatry. 2009;80(10):1075-1081.

28. Baldermann JC, Schuller T, Huys D, et al. Deep Brain Stimulation for Tourette-Syndrome: A Systematic Review and Meta-Analysis. Brain Stimulation. 2016;9(2):296-304.

29. Ackermans L, Duits A, Temel $Y$, et al. Long-term outcome of thalamic deep brain stimulation in two patients with Tourette syndrome. Journal of neurology, neurosurgery, and psychiatry. 2010;81(10):10681072.

30. Ackermans L, Duits A, van der Linden C, et al. Double-blind clinical trial of thalamic stimulation in patients with Tourette syndrome. Brain. 2011;134(Pt 3):832-844.

31. Ackermans L, Temel Y, Bauer NJ, Visser-Vandewalle V, Dutch-Flemish Tourette Surgery Study G. Vertical gaze palsy after thalamic stimulation for Tourette syndrome: case report. Neurosurgery. 2007;61(5):E1100.

32. Bajwa RJ, de Lotbiniere AJ, King RA, et al. Deep brain stimulation in Tourette's syndrome. Movement disorders. 2007;22(9):1346-1350.

33. Duits A, Ackermans L, Cath D, Visser-Vandewalle V. Unfavourable outcome of deep brain stimulation in a Tourette patient with severe comorbidity. European child \& adolescent psychiatry. 2012;21(9): 529-531.

34. Huys D, Bartsch C, Koester P, et al. Motor Improvement and Emotional Stabilization in Patients With Tourette Syndrome After Deep Brain Stimulation of the Ventral Anterior and Ventrolateral Motor Part of the Thalamus. Biological psychiatry. 2016;79(5):392-401.

35. Idris Z, Ghani AR, Mar W, et al. Intracerebral haematomas after deep brain stimulation surgery in a patient with Tourette syndrome and low factor XIIIA activity. Journal of clinical neuroscience. 2010;17(10):1343-1344.

36. Kaido T, Otsuki T, Kaneko Y, Takahashi A, Omori M, Okamoto T. Deep brain stimulation for Tourette syndrome: a prospective pilot study in Japan. Neuromodulation. 2011;14(2):123-128.

37. Kuhn J, Bartsch C, Lenartz D, et al. Clinical effectiveness of unilateral deep brain stimulation in Tourette syndrome. Translational Psychiatry. 2011;1:e52.

38. Maciunas RJ, Maddux BN, Riley DE, et al. Prospective randomized double-blind trial of bilateral thalamic deep brain stimulation in adults with Tourette syndrome. Journal of neurosurgery. 2007;107(5):10041014.

39. Marceglia S, Servello D, Foffani G, et al. Thalamic single-unit and local field potential activity in Tourette syndrome. Movement disorders. 2010;25(3):300-308.

40. Porta M, Brambilla A, Cavanna AE, et al. Thalamic deep brain stimulation for treatment-refractory Tourette syndrome: two-year outcome. Neurology. 2009;73(17):1375-1380.

41. Servello D, Porta M, Sassi M, Brambilla A, Robertson MM. Deep brain stimulation in 18 patients with severe Gilles de la Tourette syndrome refractory to treatment: the surgery and stimulation. Journal of neurology, neurosurgery, and psychiatry. 2008;79(2):136-142.

42. Smeets AY, Duits AA, Leentjens AF, et al. Thalamic Deep Brain Stimulation for Refractory Tourette Syndrome: Clinical Evidence for Increasing Disbalance of Therapeutic Effects and Side Effects at LongTerm Follow-Up. Neuromodulation. 2017.

43. Visser-Vandewalle V, Temel $Y$, Boon $P$, et al. Chronic bilateral thalamic stimulation: a new therapeutic approach in intractable Tourette syndrome. Report of three cases. Journal of neurosurgery. 2003;99(6):1094-1100.

44. Zekaj E, Saleh C, Porta M, Servello D. Temporary deep brain stimulation in Gilles de la Tourette syndrome: A feasible approach? Surgical neurology international. 2015;6:122.

45. Lee MW, Au-Yeung MM, Hung KN, Wong CK. Deep brain stimulation in a Chinese Tourette's syndrome patient. Hong Kong medical journal. 2011;17(2):147-150.

46. Okun MS, Foote KD, Wu SS, et al. A trial of scheduled deep brain stimulation for Tourette syndrome: moving away from continuous deep brain stimulation paradigms. JAMA Neurololgy. 2013;70(1):85-94. 
47. Pullen SJ, Wall CA, Lee KH, Stead SM, Klassen BT, Brown TM. Neuropsychiatric Outcome of an Adolescent Who Received Deep Brain Stimulation for Tourette's Syndrome. Case Report Neurological Medicine. 2011;2011:209467.

48. Rzesnitzek L, Wachter T, Kruger R, Gharabaghi A, Plewnia C. Suppression of extrapyramidal side effects of doxepin by thalamic deep brain stimulation for Tourette syndrome. Neurology. 2011;77(18):17081709.

49. Savica R, Stead M, Mack KJ, Lee KH, Klassen BT. Deep brain stimulation in tourette syndrome: a description of 3 patients with excellent outcome. Mayo Clinic proceedings. 2012;87(1):59-62.

50. Testini P, Zhao CZ, Stead M, Duffy PS, Klassen BT, Lee KH. Centromedian-Parafascicular Complex Deep Brain Stimulation for Tourette Syndrome: A Retrospective Study. Mayo Clinic proceedings. 2016;91(2):218-225.

51. Vernaleken I, Kuhn J, Lenartz D, et al. Bithalamical deep brain stimulation in tourette syndrome is associated with reduction in dopaminergic transmission. Biological psychiatry. 2009;66(10):e15-17.

52. Van der Linden C, Colle H, Vandewalle V, Alessi G, Rijkckaert D, de Waele L. Succesful treatment of tics with bilateral internal pallidum stimulation in a 27-year old male patient with Gilles de la Tourette syndrome. Movement disorders. 2002;17:341.

53. Dehning S, Feddersen B, Cerovecki A, Botzel K, Muller N, Mehrkens JH. Globus pallidus internus-deep brain stimulation in Tourette's syndrome: can clinical symptoms predict response? Movement disorders. 2011;26(13):2440-2441.

54. Dehning S, Leitner B, Schennach R, et al. Functional outcome and quality of life in Tourette's syndrome after deep brain stimulation of the posteroventrolateral globus pallidus internus: long-term follow-up. The world journal of biological psychiatry. 2014;15(1):66-75.

55. Dehning S, Mehrkens JH, Muller N, Botzel K. Therapy-refractory Tourette syndrome: beneficial outcome with globus pallidus internus deep brain stimulation. Movement disorders. 2008;23(9):1300-1302.

56. Diederich NJ, Kalteis K, Stamenkovic M, Pieri V, Alesch F. Efficient internal pallidal stimulation in Gilles de la Tourette syndrome: a case report. Movement disorders. 2005;20(11):1496-1499.

57. Dong S, Zhang X, Li J, Li Y. The benefits of low-frequency pallidal deep brain stimulation in a patient with Tourette syndrome. Parkinsonism \& related disorders. 2014;20(12):1438-1439.

58. Dong S, Zhuang P, Zhang XH, Li JY, Li YJ. Unilateral deep brain stimulation of the right globus pallidus internus in patients with Tourette's syndrome: two cases with outcomes after 1 year and a brief review of the literature. The Journal of International Medical Research. 2012;40(5):2021-2028.

59. Dueck A, Wolters A, Wunsch K, et al. Deep brain stimulation of globus pallidus internus in a 16-year-old boy with severe tourette syndrome and mental retardation. Neuropediatrics. 2009;40(5):239-242.

60. Hwynn N, Tagliati M, Alterman RL, et al. Improvement of both dystonia and tics with $60 \mathrm{~Hz}$ pallidal deep brain stimulation. The International journal of neuroscience. 2012;122(9):519-522.

61. Martinez-Fernandez R, Zrinzo L, Aviles-Olmos I, et al. Deep brain stimulation for Gilles de la Tourette syndrome: a case series targeting subregions of the globus pallidus internus. Movement disorders. 2011;26(10):1922-1930.

62. Shahed J, Poysky J, Kenney C, Simpson R, Jankovic J. GPi deep brain stimulation for Tourette syndrome improves tics and psychiatric comorbidities. Neurology. 2007;68(2):159-160.

63. Zhang XH, Li JY, Zhang YQ, Li YJ. Deep Brain Stimulation of the Globus Pallidus Internus in Patients with Intractable Tourette Syndrome: A 1-year Follow-up Study. Chinese Medical Journal. 2016;129(9):10221027.

64. Cannon E, Silburn P, Coyne T, O'Maley K, Crawford JD, Sachdev PS. Deep brain stimulation of anteromedial globus pallidus interna for severe Tourette's syndrome. The American journal of psychiatry. 2012;169(8):860-866

65. Gallagher CL, Garell PC, Montgomery EB, Jr. Hemi tics and deep brain stimulation. Neurology. 2006;66(3):E12.

66. Huasen B, McCreary R, Evans J, Potter G, Silverdale M. Cervical myelopathy secondary to Tourette's syndrome managed by urgent deep brain stimulation. Movement disorders. 2014;29(4):452-453. 
67. Kefalopoulou Z, Zrinzo L, Jahanshahi M, et al. Bilateral globus pallidus stimulation for severe Tourette's syndrome: a double-blind, randomised crossover trial. Lancet neurology. 2015;14(6):595-605.

68. Massano J, Sousa C, Foltynie T, Zrinzo L, Hariz M, Vaz R. Successful pallidal deep brain stimulation in 15year-old with Tourette syndrome: 2-year follow-up. Journal of neurology. 2013.

69. Sachdev PS, Mohan A, Cannon E, et al. Deep brain stimulation of the antero-medial globus pallidus interna for Tourette syndrome. PloS one. 2014;9(8):e104926.

70. Smeets $A Y$, Duits $A A$, Plantinga $B R$, et al. Deep Brain Stimulation of the internal globus pallidus in refractory Tourette Syndrome. Clinical Neurology and Neurosurgery. 2016;142:54-59.

71. Welter ML, Houeto JL, Thobois S, et al. Anterior pallidal deep brain stimulation for Tourette's syndrome: a randomised, double-blind, controlled trial. Lancet neurology. 2017.

72. Piedimonte F, Andreani JC, Piedimonte L, et al. Behavioral and motor improvement after deep brain stimulation of the globus pallidus externus in a case of Tourette's syndrome. Neuromodulation. 2013;16(1):55-58.

73. Burdick A, Foote KD, Goodman W, et al. Lack of benefit of accumbens/capsular deep brain stimulation in a patient with both tics and obsessive-compulsive disorder. Neurocase. 2010;16(4):321-330.

74. Kuhn J, Lenartz D, Mai JK, et al. Deep brain stimulation of the nucleus accumbens and the internal capsule in therapeutically refractory Tourette-syndrome. Journal of neurology. 2007;254(7):963-965.

75. Neuner I, Podoll K, Lenartz D, Sturm V, Schneider F. Deep brain stimulation in the nucleus accumbens for intractable Tourette's syndrome: follow-up report of 36 months. Biological psychiatry. 2009;65(4).

76. Zabek M, Sobstyl M, Koziara H, Dzierzecki S. Deep brain stimulation of the right nucleus accumbens in a patient with Tourette syndrome. Case report. Neurologia i neurochirurgia polska. 2008;42(6):554-559.

77. Flaherty AW, Williams ZM, Amirnovin R, et al. Deep brain stimulation of the anterior internal capsule for the treatment of Tourette syndrome: technical case report. Neurosurgery. 2005;57:E403.

78. Martinez-Torres I, Hariz MI, Zrinzo L, Foltynie T, Limousin P. Improvement of tics after subthalamic nucleus deep brain stimulation. Neurology. 2009;72(20):1787-1789.

79. Ackermans L, Temel Y, Cath D, et al. Deep brain stimulation in Tourette's syndrome: two targets? Movement disorders. 2006;21(5):709-713.

80. Hauseux PA, Cyprien F, Cif L, et al. Long-term follow-up of pallidal Deep Brain Stimulation in teenagers with refractory Tourette syndrome and comorbid psychiatric disorders: About three cases. Journal of the European Paediatric Neurology Society. 2016.

81. Houeto JL, Karachi C, Mallet L, et al. Tourette's syndrome and deep brain stimulation. Journal of neurology, neurosurgery, and psychiatry. 2005;76(7):992-995.

82. Motlagh MG, Smith ME, Landeros-Weisenberger A, et al. Lessons Learned from Open-label Deep Brain Stimulation for Tourette Syndrome: Eight Cases over 7 Years. Tremor and other hyperkinetic movements. 2013;3.

83. Porta M, Servello D, Zanaboni C, et al. Deep brain stimulation for treatment of refractory Tourette syndrome: long-term follow-up. Acta neurochirurgica. 2012;154(11):2029-2041.

84. Servello D, Sassi M, Brambilla A, Defendi S, Porta M. Long-term, post-deep brain stimulation management of a series of 36 patients affected with refractory gilles de la tourette syndrome. Neuromodulation. 2010;13(3):187-194.

85. Servello D, Sassi M, Brambilla A, et al. De novo and rescue DBS leads for refractory Tourette syndrome patients with severe comorbid OCD: a multiple case report. Journal of neurology. 2009;256(9):15331539.

86. Shields DC, Cheng ML, Flaherty AW, Gale JT, Eskandar EN. Microelectrode-guided deep brain stimulation for Tourette syndrome: within-subject comparison of different stimulation sites. Stereotactic and functional neurosurgery. 2008;86(2):87-91.

87. Welter ML, Mallet L, Houeto JL, et al. Internal pallidal and thalamic stimulation in patients with Tourette syndrome. Archives of neurology. 2008;65(7):952-957.

88. Servello D, Zekaj E, Saleh C, Lange N, Porta M. Deep Brain Stimulation in Gilles de la Tourette Syndrome: What Does the Future Hold? A Cohort of 48 Patients. Neurosurgery. 2016;78(1):91-100. 
Chapter 1

89. Hassler R, Dieckmann G. [Stereotaxic treatment of tics and inarticulate cries or coprolalia considered as motor obsessional phenomena in Gilles de la Tourette's disease]. Revue neurologique. 1970;123(2):89100.

90. Fraint A, Pal G. Deep Brain Stimulation in Tourette's Syndrome. Frontiers in neurology. 2015;6:170. 


\section{Chapter 2}

The role of the basal ganglia in Tourette Syndrome

A.Y.J.M Smeets, A.F.G Leentjens, A.A Duits, Y Temel, L Ackermans In preparation 

In Tourette Syndrome (TS) dysfunction of the basal ganglia (BG) and the connected cortico-basal-ganglia-thalamocortical (CBGT) pathways plays a major role in the underlying pathophysiology, although the exact mechanisms are not fully understood ${ }^{1}$. Knowledge about the basic functional anatomy of the BG and their pathways is essential in understanding the mechanisms and effects of Deep Brain Stimulation (DBS) in TS patients. Therefore, we provide a general anatomical overview of the BG and their pathways, followed by an exploration of the changes in the CBGT circuits in TS patients, and an explanation of the mechanisms of DBS.

\section{ANATOMY OF THE BASAL GANGLIA}

The BG are a group of interconnected deep subcortical nuclei which are located symmetrically across the cerebral hemispheres and consist of (Figure 1) ${ }^{2}$ :

- The putamen and nucleus caudatus, together called the striatum.

- The subthalamic nucleus (STN).

- The external globus pallidus (GPe).

- The substantia nigra, pars compacta (SNC).

- The internal globus pallidus (GPi): divided in a posterolateral part, the motor area (posterior GPi), and an anteromedial part, the limbic area (anterior GPi).

- The substantia nigra, pars reticulata (SNr).

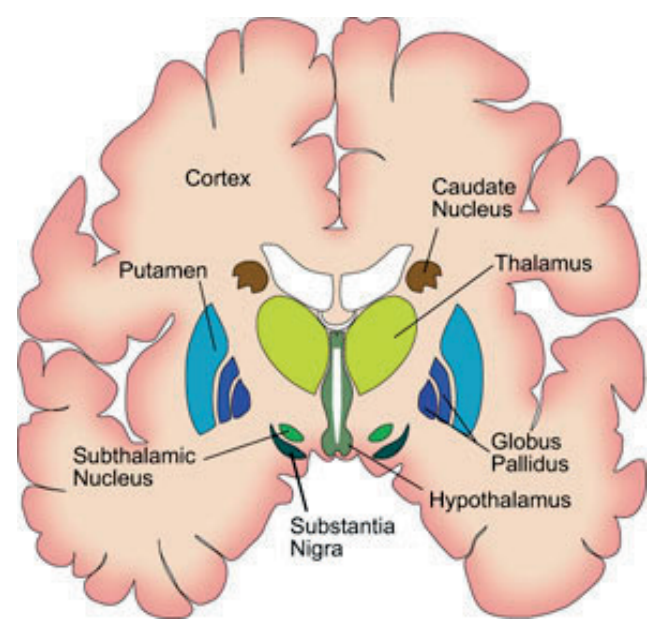

Figure 1: Anatomy of the basal ganglia.

The cortex sends information to the BG, were it is processed and sent back to the cortex via the thalamus (Figure 2). The striatum and the STN are the primary input structures of the BG. The striatum receives excitatory input from all areas of the cerebral cortex and the STN from motor areas in the frontal lobe. The striatum also receives substantial 
afferents from the SNc and the intralaminar thalamic nuclei (mainly the centromedianparafascicular (Cm-Pf) complex and the ventrolateral thalamic nuclei (VL)), in addition to intrastriatal inputs ${ }^{2-4}$.

The GPe and SNc are intrinsic nuclei within the BG. The GPe connects the input and output stations and receives excitatory input from the STN and inhibitory input from the striatum. The GPe in turn sends inhibitory output to STN, the GPi and the SNr. The SNc sends inhibitory as well as excitatory output back to the striatum to modulate the function of the $B G$ via dopamine (DA) ${ }^{2-4}$.

The GPi and SNr are the primary output structures of the BG and they are a single cellular structure divided by the internal capsule. These output nuclei receive fast, excitatory input from the STN and slower, inhibitory input from the striatum. The GPi and SNr in turn sent inhibitory signals predominantly to the thalamus, which then sends excitatory signals back to the cortex. Thalamic area's innervated by the BG are the ventro-anterior nucleus (VA), the mediodorsal nucleus (MD), the VL nuclei and the CmPf complex ${ }^{2-4}$.

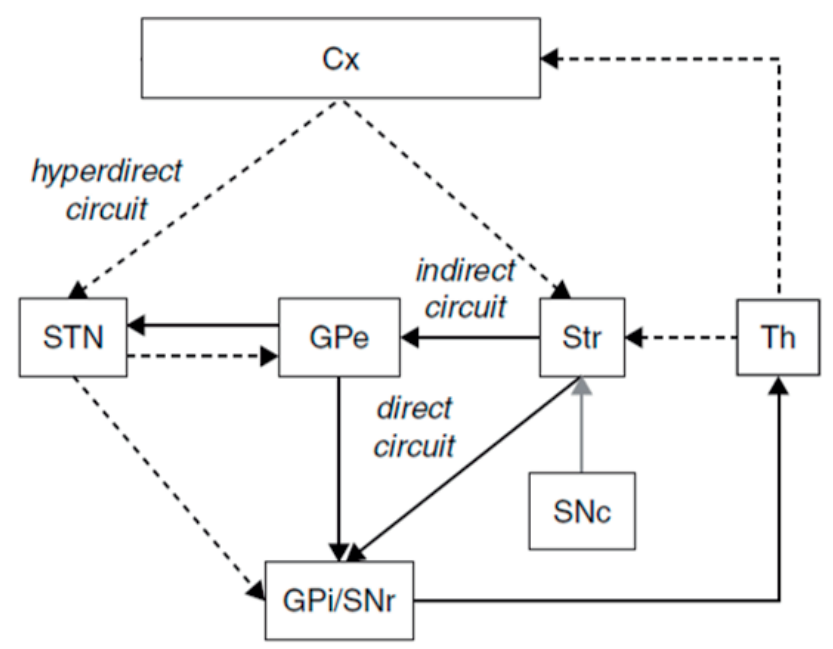

\section{$\rightarrow$ glutamate $\longrightarrow$ GABA $\longrightarrow$ dopamine}

Figure 2: Schematic representation of the cortico-basal-ganglia-thalamocortical pathways.

Abbreviations: Cx: cerebral cortex; STN: subthalamic nucleus; GPe: external globus pallidus; Str: striatum; Th: thalamus; GPi: internal globus pallidus; SNr: substantia nigra, pars reticulata; SNc: substantia nigra, pars compacta; GABA: Gamma-aminobutyric acid. Figure adapted from book chapter written by A.F.G Leentjens. 


\section{CORTICO-BASAL-GANGLIA-THALAMOCORTICAL PATHWAYS}

Three CBGT pathways have been described (Figure 2), however, anatomical evidence points out that this intrinsic circuitry is far more complex ${ }^{1,5}$ :

- The direct pathway, which is a positive feedback loop. In this pathway the cortex sends excitatory signals to the striatum, which inhibits the GPi/SNr complex. These two structures then disinhibit the thalamus, thereby effectively reinforcing the excitatory signals directed back to the cortex.

- The indirect pathway constitutes a negative feedback loop. In this pathway, signals from the striatum are redirected to inhibit the GPe. These signals then disinhibit the STN and GPi/SNr complex, which leads to more inhibition of the thalamus and its projections to the cortex.

- The hyperdirect pathway also constitutes a negative feedback loop. The striatum is bypassed and signals from the cortex are directly projected onto the STN to excite the GPi/SNr complex, thereby inhibiting the thalamus and its projections to the cortex.

In general, the direct pathway facilitates movement and the indirect and hyperdirect pathways inhibit movement. Via the fast conducting hyperdirect pathway the thalamus is initially inhibited, it is then subsequently disinhibited by the direct pathway, with the indirect pathway finally exerting inhibition once more. This arrangement allows for precise selection of action, with a circumscribed initiation and termination. The direct pathway activates desired programs while the hyperdirect and indirect pathways suppress unwanted competing programs. Therefore, selected movements are enabled and competing postures and movements are prevented from interfering with the one selected $^{3-5}$.

The CBGT projections can be divided into five important functional circuits, named for their cortical areas of origin and action: 1) the motor, 2) the associative, 3) the limbic, 4) the oculomotor and 5) the orbitofrontal circuit ${ }^{6}$. The first three circuits seem to be involved in the pathophysiology of TS and are shown in Figure 3. The main cortical inputs to the motor circuit are the primary motor, premotor, supplementary motor (SMA) and somatosensory cortex and signals are sent to the putamen. The main inputs to the oculomotor, associative and orbitofrontal circuit are the dorsolateral prefrontal and lateral orbitofrontal cortex and signals are sent mainly to the nucleus caudatus. Information from these four circuits is conveyed primarily to the posterior GPi/SNr. The main inputs to the limbic circuit are the anterior cingulate and orbitofrontal cortex, the hippocampus, and the amygdala and signals enter via the ventral striatum. The ventral striatum contains the nucleus accumbens (NA), the ventromedial part of the nucleus caudatus, and the medial part of the tuberculum olfactorium. Limbic information is conveyed to the anterior $\mathrm{GPi}^{2-4,6}$. 
There is a parallel organisation of these functionally segregated CBGT circuits. Each circuit receives multiple, partially overlapping corticostriatal inputs, which are progressively integrated in their passage through the BG to a restricted portion of the thalamus, and from there back to a single cortical area. Each circuit is partially closed by the restricted thalamocortical projection returned to one of that circuit's multiple sources of corticostriatal inputs ${ }^{6}$.
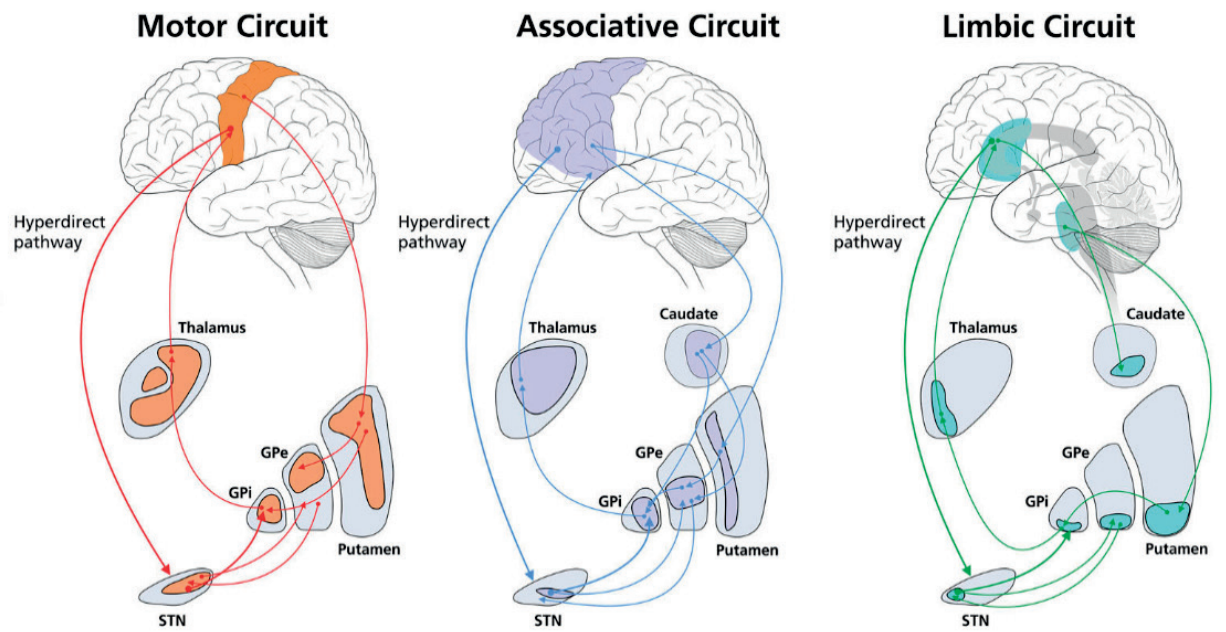

Figure 3: Anatomical-functional organization of the circuits of the cortico-basal-ganglia-thalamocortical pathways.

Re-entrant circuits from functionally and anatomically distributed, parallel circuits which integrate to perform massive processing in different brain areas. In Tourette Syndrome disturbances are observed in motor, associative and limbic functions and those circuits are shown here.

Abbreviations: GPe: external globus pallidus; GPi: internal globus pallidus; STN: subthalamic nucleus.

Figure adapted from article by Hashemiyoon et al $^{7}$.

\section{BASAL GANGLIA DYSFUNCTION IN TOURETTE SYNDROME}

Structural changes have been reported in the BG and in a broad range of connected motor and limbic cortical areas of TS patients ${ }^{8-10}$. Various neuroimaging studies have shown reduced volumes and abnormal asymmetries of the nucleus caudatus, putamen and globus pallidus ${ }^{8,11-13}$. Volumes of grey matter are abnormal ${ }^{9}$ and white matter analysis show widespread abnormalities in patients with TS ${ }^{14,15}$. Neuropathological studies have shown structural changes in neurons in the striatum and globus pallidus of TS patients ${ }^{16,17}$. Morphological changes have also been shown in a broad range of limbic and motor cortical areas, including increased and/or decreased volumes of specific brain areas, decreased grey matter volumes and cortical thickness ${ }^{9}$. Moreover, abnormalities in the dopaminergic neurotransmitter system seem to play a significant 
role ${ }^{18-21}$. Dopaminergic neurons in the SNc have contrasting effects on the facilitating en inhibiting pathways since they tend to activate striatal neurons contributing to the direct pathway (D1-receptors) and inhibit striatal neurons in the indirect pathway (D2receptors). The overall impact of DA on the $B G$ is the enhancement of thalamocortical excitation ${ }^{2}$. Several functional neuroimaging studies have shown abnormalities in DA transporter and DA binding in the striatum of TS patients ${ }^{18-21}$.

Traditionally, TS has been considered a member of the hyperkinetic movement disorder family. They all express an excess of movement with uncontrollable and relatively rapid motor acts and share a common pharmacology, since they are suppressed by DA antagonists. Examples are chorea and athetosis (e.g. due to Huntington's disease), ballism (e.g. due to STN infarction) and tics (e.g. due to TS). Functional models of the BG have suggested potential mechanisms for hyperkinetic movement disorders. They view all hyperkinetic movement disorders as one entity and assume that they have common underlying properties and they have centred on the existence of abnormalities in the motor circuits ${ }^{14}$. The 'box and arrow' model depicts the global neuronal activity modulation of whole brain areas (boxes) along the CBGT pathway as a function of their connectivity (arrows). In this model, hyperkinetic disorders are explained by striatal disinhibition that leads to reduced tonic inhibition exerted by the GPi/SNr on its thalamocortical targets. The 'action selection' model accounts for the local changes in the inhibition of neuronal sub-groups within the output nuclei. In this model, hyperkinetic symptoms originate from abnormal focal activation of striatal neurons which inhibit the activity of a subpopulation of GPi neurons ${ }^{1,5,14}$.

In TS, this unwanted inhibition of the main output nuclei of the BG leads to disinhibition of its thalamocortical targets and this in turn can lead to repeated stereotyped unwanted movements like tics (Figure 4) ${ }^{14}$. Mechanisms that can be responsible for such an unwanted activation are excessive cortical or thalamic input to the striatum, deficiency of intrastriatal inhibition, abnormal DA transmission, and abnormal membrane excitability. Many features of TS can be explained as a failure to inhibit unwanted movements, behaviours, and thoughts, resulting from these abnormal BG output patterns. For example, abnormal activation of the motor cortex can produce simple tics, abnormal activation of the premotor cortex or SMA can lead to complex tics, abnormal activation of the orbitofrontal cortex can cause compulsions, and abnormal activation of the dorsolateral prefrontal cortex can produce attention deficits 14 


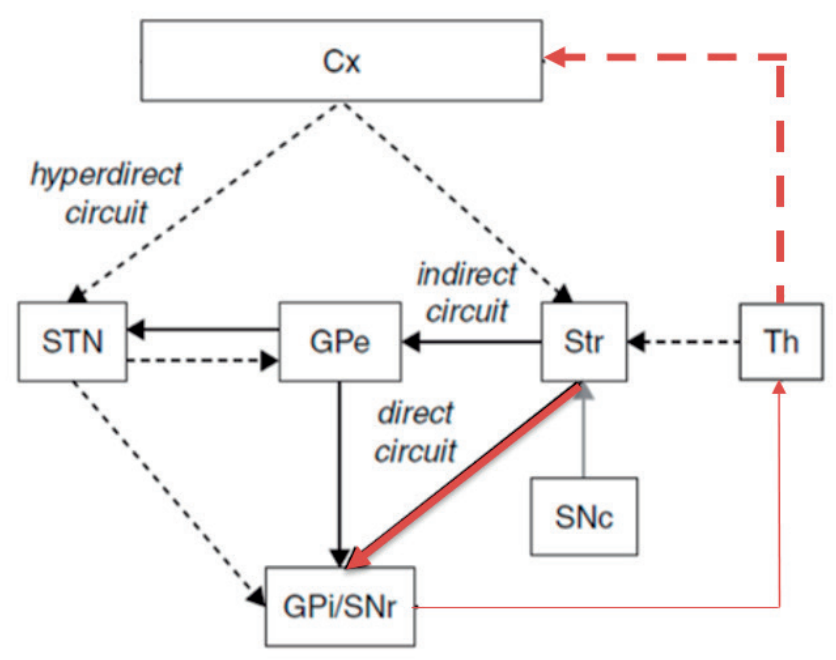

\section{$\longrightarrow$ glutamate $\longrightarrow$ GABA $\longrightarrow$ dopamine}

Figure 4: Schematic representation of the abnormalities in the cortico-basal-ganglia-thalamocortical pathways in Tourette Syndrome. (red lines: fat line = enhanced activity, thin line = reduced activity).

Disinhibition within the striatum leads to excessive inhibition of the GPi/SNr in the direct circuit, which in turn leads to less inhibition of the thalamus and as a consequence excessive excitation of the cortical targets. Abbreviations: Cx: cerebral cortex; STN: subthalamic nucleus; GPe: external globus pallidus; Str: striatum; Th: thalamus; GPi: internal globus pallidus; SNr: substantia nigra, pars reticulata; SNc: substantia nigra, pars compacta; GABA: Gamma-aminobutyric acid. Figure adapted from book chapter written by A.F.G Leentjens.

\section{ELECTROPHYSIOLOGY IN TOURETTE SYNDROME}

Animal models of TS using electrophysiological recordings are used to better understand the underlying neuronal mechanisms of the disease. Focal striatal disinhibition using microinjections of Gamma-aminobutyric acid-A $\left(G A B A_{A}\right)$ antagonists leads to the appearance of motor and vocal tics in non-human primates ${ }^{22-24}$ and motor tics in rodents ${ }^{25,26}$. These animals transiently display sudden, rapid, and repetitive movements that resemble the simple motor tics of TS patients. Both phasic increases and decreases in firing rate were found in GPi and GPe neurons in relation to the tics ${ }^{22-}$ 24. DBS targeting different areas within the BG and the thalamus provides a rare opportunity to directly observe neuronal activity in TS patients. Intra-operative recordings from drivable microelectrodes present the opportunity for single unit recordings, while post-operative recordings of local field potentials (LFP) can be made from implanted macroelectrodes ${ }^{7}$. Neurophysiological analysis of intra- and postoperative human electrophysiological recordings from clinical DBS studies suggest a link 
between tic behaviour and activity in both the thalamus and the GPi ${ }^{7,27}$. Single unit recordings in the trajectory targeting the nucleus ventro-oralis internus (Voi) of the thalamus showed that it is characterized by a localized pattern of neuronal bursting ${ }^{28}$. However, these findings were also seen during DBS under general anaesthesia for other indications and are neither unique to the target or to the disorder. Extracellular electrophysiological recordings performed during pallidotomy surgery revealed a decreased overall firing rate in the posterior GPi of TS patients ${ }^{29}$. In addition to the overall change, a similar bursting activity was seen and almost half of these GPi neurons showed tic-related neuronal changes around the time of the tic. We found comparable results of a reduced baseline firing rate and tic dependent transient rate changes when targeting the anterior GPi in TS patients ${ }^{27}$. Only one study has performed chronic LFP recordings from implanted thalamic electrodes and correlated these findings with the symptoms. An increase in spectral activity in gamma band power and theta/gamma cross frequency was found in responders of thalamic DBS ${ }^{30}$.

\section{RATIONALE FOR TARGETING THE THALAMUS AND THE INTERNAL GLOBUS PALLIDUS}

The reason for choosing a certain target for DBS in refractory TS patients is based on the focus of the treatment, for example the motor aspects of tics or the compulsive and behavioural elements. A systematic review and meta-analysis from 2016 identified 156 reported cases of TS DBS, of which 78 targeted the thalamus, 44 the anterior GPi, and 20 the posterior $\mathrm{GPi}^{31}$. Other targets have been less studied. The thalamus and the GPi are key structures within the CBGT pathways (Figure 2 and 3) and by influencing them through high frequency stimulation the signalling throughout the BG can be adapted. The main goal of DBS is to restore the imbalance present in the CBGT pathways of TS patients and to relieve symptoms. The thalamus is not only the target of integrated BG output and the most important relay station to the cortex, it also projects back to the striatum, and controls the information transmitted to both the cortex and the striatum. The GPi is the primary output nucleus of the BG, and stimulation of this nucleus can modulate the whole CBGT circuit. Moreover, the thalamus and the GPi seem to play a significant role in the underlying pathophysiology of TS ${ }^{1,14}$, which is supported by clinical evidence showing that the degree of tic improvement is most robust for these targets ${ }^{31}$.

The first DBS target used in TS was the medial thalamus and the selection was motivated by thalamotomies performed in 1970 by Hassler and Dieckmann ${ }^{32}$. They reported on the beneficial effects of lesioning the intralaminar and midline thalamic nuclei in patients suffering from TS, and, in patients with facial tics, also the Voi. The difficulty was that they made up to ten coagulations in each thalamus, so a strategic point had to be found to stimulate as many of the nuclei targeted with one electrode 
for each hemisphere. The strategic point chosen was located at the cross point between the anteromedial border of the centromedian nucleus $(\mathrm{Cm}$, as part of the intralaminar thalamic nuclei), the substantia periventricularis (Spv, as part of the medial thalamic nuclei), and the Voi (as part of the ventrolateral thalamic nuclei). This target has been slightly adapted by others, some stimulate a little bit more anterior while others stimulate the centre of the Cm-Pf complex. It is hypothesized that targeting this area would lead to modulation of motor symptoms via stimulation of the $\mathrm{Cm}$ (motor circuit), modulation of behavioural symptoms via stimulation of the Spv (projections to the ventral striatum, part of the limbic circuit), and modulation of facial tics via the Voi (direct projections to facial parts of the (pre)motor cortex) ${ }^{33}$.

In 2002 the first TS patient was treated with DBS of the GPi ${ }^{34}$ and several years later this area gained more interest as a potential target for TS. The GPi can be divided in a motor area (posterior GPi) and a limbic area (anterior GPi). The posterior GPi became a readily accepted target given the extensive evidence of the efficacy of this target in other hyperkinetic movement disorders, specifically dyskinesia's in Parkinson's disease ${ }^{35}$ and dystonia ${ }^{36}$. These disorders share some underlying mechanisms, but they only centre on the existence of abnormalities in the motor circuits of the BG. It was thought that targeting the posterior GPi would lead to modulation of motor symptoms in TS, but not necessarily to modulation of behavioural symptoms. Recently, more attention is being paid on the role of the associative and limbic circuitry in TS, and it is even thought that motor symptoms of TS can result from abnormal motivational drives from these circuits rather than from dysfunction of the motor circuit ${ }^{1,12}$. Therefore, it is hypothesized that targeting the anterior GPi might improve both behavioural and motor symptoms of TS and result in even more clinical benefits.

\section{MECHANISMS OF DEEP BRAIN STIMULATION}

How does DBS influence the electrophysiological signals in the CBGT circuits and restore the imbalance present in TS patients? Evidence shows that DBS exerts its effect by causing functional ablation, which reduces information flow through the stimulated nucleus ${ }^{20,29}$. High frequency stimulation inhibits the targeted area because the negative extracellular charge makes it more difficult to generate action potentials. The similarity between findings in Parkinson patients, TS patients and normal animals supports the notion that the mechanisms of DBS are comparable regardless of the underlying disease or the region of stimulation ${ }^{20,37}$. In TS there seems to be a disbalance in the CBGT circuit as shown in Figure 4. In summary this means that disinhibition within the striatum leads to excessive inhibition of the GPi/SNr complex in the direct circuit, which in turn leads to disinhibition of the thalamus and therefore to enhanced excitation of the cortical targets. DBS of the thalamus can correct this disinhibition by causing functional ablation and reduced signalling through the thalamus, leading to less 
excitation of the cortical targets. Moreover, the afferent enhancing projections from several thalamic nuclei (e.g. the $\mathrm{Cm}$-Pf complex) to the striatum might be inhibited by the stimulation. DBS of the GPi is more difficult to be explained by the above-mentioned working mechanisms. It is thought that stimulation of the GPi results in partial temporal blocking of neuronal responses to stimulation pulses. This abolishes the tic-related activity of the neurons and for a short period increases the overall firing rate of the nucleus ${ }^{20}$. If the firing rate of the GPi during stimulation is increased, this leads to more inhibition of the thalamus and as a result to less excitation of the cortical targets. Studies of long-term electrophysiological changes underlying the progressive effects of DBS in TS are unfortunately still lacking.

\section{CONCLUSION}

Although dysfunction of the CBGT pathways seems to play a critical role in the underlying pathophysiology of TS, the exact mechanisms are still unknown. The emerging evidence that TS involves CBGT dysfunction emphasizes that further investigation of the basic neurobiology of the BG is likely to be indispensable for improved understanding of the pathophysiology of TS. Moreover, this might have direct consequences for choosing DBS targets and for treating refractory TS patients. 


\section{REFERENCES}

1. Albin RL, Mink JW. Recent advances in Tourette syndrome research. Trends in neurosciences. 2006;29(3):175-182.

2. Utter AA, Basso MA. The basal ganglia: an overview of circuits and function. Neuroscience and biobehavioral reviews. 2008;32(3):333-342.

3. Albin RL, Young $A B$, Penney JB. The functional anatomy of basal ganglia disorders. Trends in neurosciences. 1989;12(10):366-375.

4. Mink JW. The basal ganglia: focused selection and inhibition of competing motor programs. Progress in neurobiology. 1996;50(4):381-425.

5. DeLong MR. Primate models of movement disorders of basal ganglia origin. Trends in neurosciences. 1990;13(7):281-285.

6. Alexander GE, DeLong MR, Strick PL. Parallel organization of functionally segregated circuits linking basal ganglia and cortex. Annual Review of Neuroscience. 1986;9:357-381.

7. Hashemiyoon R, Kuhn J, Visser-Vandewalle V. Putting the Pieces Together in Gilles de la Tourette Syndrome: Exploring the Link Between Clinical Observations and the Biological Basis of Dysfunction. Brain Topography. 2016.

8. Bloch MH, Leckman JF, Zhu H, Peterson BS. Caudate volumes in childhood predict symptom severity in adults with Tourette syndrome. Neurology. 2005;65(8):1253-1258.

9. Peterson BS, Thomas P, Kane MJ, et al. Basal Ganglia volumes in patients with Gilles de la Tourette syndrome. Archives of general psychiatry. 2003;60(4):415-424.

10. Rauch SL, Whalen PJ, Curran T, et al. Probing striato-thalamic function in obsessive-compulsive disorder and Tourette syndrome using neuroimaging methods. Advances in neurology. 2001;85:207-224.

11. Eidelberg D, Moeller JR, Antonini A, et al. The metabolic anatomy of Tourette's syndrome. Neurology. 1997;48(4):927-934.

12. Peterson BS, Skudlarski $P$, Anderson AW, et al. A functional magnetic resonance imaging study of tic suppression in Tourette syndrome. Archives of general psychiatry. 1998;55(4):326-333.

13. Singer HS. Neurobiology of Tourette syndrome. Neurologic clinics. 1997;15(2):357-379.

14. Mink JW. Basal ganglia dysfunction in Tourette's syndrome: a new hypothesis. Pediatric neurology. 2001;25(3):190-198.

15. Worbe $\mathrm{Y}$, Malherbe C, Hartmann A, et al. Functional immaturity of cortico-basal ganglia networks in Gilles de la Tourette syndrome. Brain. 2012;135(Pt 6):1937-1946.

16. Kalanithi PS, Zheng W, Kataoka Y, et al. Altered parvalbumin-positive neuron distribution in basal ganglia of individuals with Tourette syndrome. Proceedings of the National Academy of Sciences of the United States of America. 2005;102(37):13307-13312.

17. Kataoka Y, Kalanithi PS, Grantz H, et al. Decreased number of parvalbumin and cholinergic interneurons in the striatum of individuals with Tourette syndrome. The Journal of comparative neurology. 2010;518(3):277-291.

18. Ernst M, Zametkin AJ, Jons PH, Matochik JA, Pascualvaca D, Cohen RM. High presynaptic dopaminergic activity in children with Tourette's disorder. Journal of the American Academy of Child and Adolescent Psychiatry. 1999;38(1):86-94.

19. Singer HS, Szymanski S, Giuliano J, et al. Elevated intrasynaptic dopamine release in Tourette's syndrome measured by PET. The American journal of psychiatry. 2002;159(8):1329-1336.

20. Israelashvili M, Loewenstern Y, Bar-Gad I. Abnormal neuronal activity in Tourette syndrome and its modulation using deep brain stimulation. Journal of neurophysiology. 2015;114(1):6-20.

21. Ackermans L, Temel Y, Visser-Vandewalle V. Deep brain stimulation in Tourette's Syndrome. Neurotherapeutics. 2008;5(2):339-344.

22. Bronfeld M, Belelovsky K, Bar-Gad I. Spatial and temporal properties of tic-related neuronal activity in the cortico-basal ganglia loop. The Journal of neuroscience. 2011;31(24):8713-8721. 
23. McCairn KW, Bronfeld M, Belelovsky K, Bar-Gad I. The neurophysiological correlates of motor tics following focal striatal disinhibition. Brain. 2009;132(Pt 8):2125-2138.

24. McCairn KW, Nagai Y, Hori Y, et al. A Primary Role for Nucleus Accumbens and Related Limbic Network in Vocal Tics. Neuron. 2016;89(2):300-307.

25. Bronfeld M, Israelashvili M, Bar-Gad I. Pharmacological animal models of Tourette syndrome. Neuroscience and biobehavioral reviews. 2013;37(6):1101-1119.

26. Pogorelov V, Xu M, Smith HR, Buchanan GF, Pittenger C. Corticostriatal interactions in the generation of tic-like behaviors after local striatal disinhibition. Experimental neurology. 2015;265:122-128.

27. Israelashvili $\mathrm{M}$, Smeets $\mathrm{A}$, Bronfeld $\mathrm{M}$, et al. Tonic and phasic changes in anteromedial globus pallidus activity in Tourette syndrome. Movement disorders. 2017.

28. Marceglia S, Servello D, Foffani G, et al. Thalamic single-unit and local field potential activity in Tourette syndrome. Movement disorders. 2010;25(3):300-308.

29. Zhuang $P$, Hallett M, Zhang $X$, Li J, Zhang Y, Li Y. Neuronal activity in the globus pallidus internus in patients with tics. Journal of neurology, neurosurgery, and psychiatry. 2009;80(10):1075-1081.

30. Maling N, Hashemiyoon R, Foote KD, Okun MS, Sanchez JC. Increased thalamic gamma band activity correlates with symptom relief following deep brain stimulation in humans with Tourette's syndrome. PloS one. 2012;7(9):e44215.

31. Baldermann JC, Schuller T, Huys D, et al. Deep Brain Stimulation for Tourette-Syndrome: A Systematic Review and Meta-Analysis. Brain Stimulation. 2016;9(2):296-304.

32. Hassler R, Dieckmann G. [Stereotaxic treatment of tics and inarticulate cries or coprolalia considered as motor obsessional phenomena in Gilles de la Tourette's disease]. Revue neurologique. 1970;123(2):89100.

33. Vandewalle V, van der Linden C, Groenewegen HJ, Caemaert J. Stereotactic treatment of Gilles de la Tourette syndrome by high frequency stimulation of thalamus. Lancet. 1999;353(9154):724.

34. C vdL, H C, Vandewalle V, G A, D R, L dW. Succesful treatment of tics with bilateral internal pallidum stimulation in a 27-year old male patient with Gilles de la Tourette syndrome. Movement disorders. 2002;17:341.

35. Anderson VC, Burchiel KJ, Hogarth P, Favre J, Hammerstad JP. Pallidal vs subthalamic nucleus deep brain stimulation in Parkinson disease. Archives of neurology. 2005;62(4):554-560.

36. Coubes P, Roubertie A, Vayssiere N, Hemm S, Echenne B. Treatment of DYT1-generalised dystonia by stimulation of the internal globus pallidus. Lancet. 2000;355(9222):2220-2221.

37. Levy R, Hutchison WD, Lozano AM, Dostrovsky JO. Synchronized neuronal discharge in the basal ganglia of parkinsonian patients is limited to oscillatory activity. The Journal of neuroscience. 2002;22(7):28552861. 



\section{Chapter 3}

Thalamic Deep Brain Stimulation for refractory Tourette Syndrome:

clinical evidence for increasing disbalance of therapeutic effects and side effects at long-term follow-up

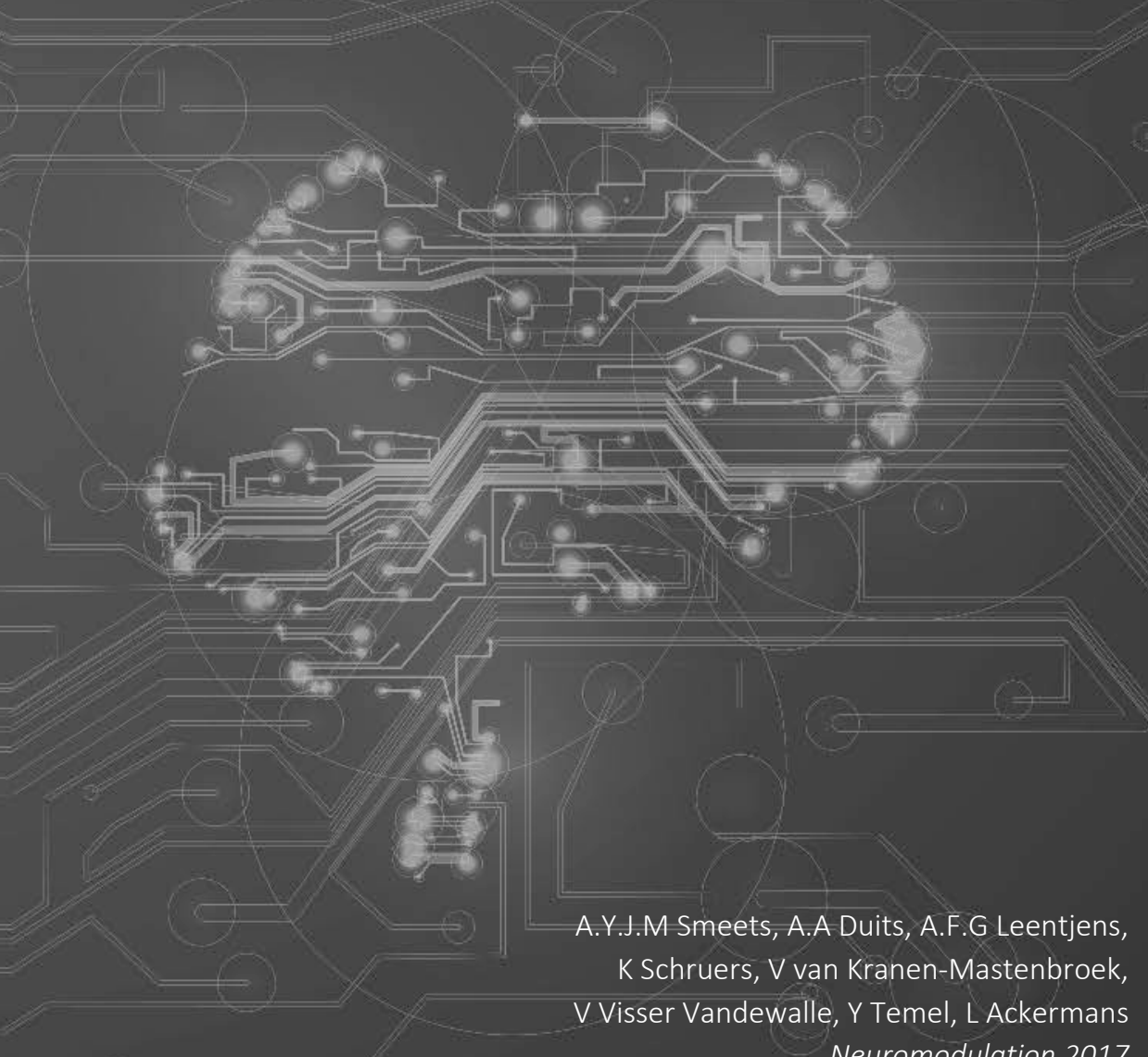




\section{ABSTRACT}

Objective: Thalamic Deep Brain Stimulation is effective in reducing tics in patients with refractory Tourette syndrome at the short-term. Here, we report on the long-term outcome.

Methods: Seven patients underwent bilateral Deep Brain Stimulation between 2001 and 2008. The target was the centromedian nucleus, substantia periventricularis and nucleus ventro-oralis internus cross point of the thalamus. The effect on tics and side effects were evaluated with a variable follow-up duration of 12 to 78 months.

Results: Patient 1 and 2 showed good tic improvements of 81.6\% (60 months) and 50\% (36 months), respectively. However, side effects like reducing levels of energy and visual disturbances increased. In patient 1 the target was changed to the anterior part of internal globus pallidus and patient 2 switched the stimulator permanently off. Patient 3 experiences still satisfying results with a tic improvement of $88.9 \%$ (78 months). Patient 4 and 7 showed minor tic improvements of 34\% (16 months) and 9\% (60 months), respectively. In both patient's side effects became more severe and the target was changed to the anterior part of the internal globus pallidus. Patient 5 showed a tic improvement of $27.5 \%$ (12 months) and went abroad for stimulation of the external globus pallidus. Patient 6 developed cerebellar atrophy. He experienced several nonstimulation related side effects and turned the stimulator off.

Conclusions: There seems to be an increasing disbalance of therapeutic effects and side effects at long-term follow-up, often leading to either switching the stimulator off or new surgery with a different neuro-anatomical target. 


\section{INTRODUCTION}

Tourette Syndrome (TS) is a childhood-onset neurodevelopmental disorder characterized by multiple motor and vocal tics ${ }^{1,2}$. Although symptoms usually subside by adulthood, a significant proportion of patients fail to respond to standard medical and behavioural therapies ${ }^{3-5}$. For these patients, Deep Brain Stimulation (DBS) has emerged as a therapeutic escalation. At present, six different targets have been stimulated ${ }^{6,7}$; the medial part of the thalamus, the internal globus pallidus (GPi), the external globus pallidus (GPe), the nucleus accumbens (NA), the anterior limb of internal capsule (ALIC), and the subthalamic nucleus (STN). Most of the TS patients have shown beneficial shortterm effects following DBS. Overall, DBS resulted in a significant short-term improvement of $53 \%$ on the Yale Global Tic Severity Scale (YGTSS) ${ }^{7,8}$. In general, the degree of tic improvement appeared to be most robust for the thalamic and the GPi targets ${ }^{7}$.

So far, long-term follow-up data are sparse. Porta et al ${ }^{9}$ described their six years follow-up of thalamic DBS in 18 TS patients and showed a significant improvement on tics and comorbid behavioural disorders. However, long-term complications and difficulties such as noncompliance and differences in opinions between the medical team and the patients as to their satisfaction with the outcome were reported ${ }^{9}{ }^{10}$. Motlagh et al ${ }^{11}$ reported their experiences with DBS of the medial thalamus $(n=5)$ or the GPi $(n=3)$ in eight TS patients over seven years. Only three patients experienced more than $50 \%$ tic reduction (thalamic target in all three patients) and two patients had their DBS leads removed, one due to an infection and one to due lack of effect (thalamic target in both patients).

Given the small amount of published data, it is difficult to consider the gain for future individual TS patients. Therefore, rigorous reporting of all available data, and especially long-term follow-up data, is highly needed. The present paper reports on the long-term outcome of seven refractory TS patients treated with DBS of the medial thalamus between 2001 and 2008. The initial one-year outcome of six patients ${ }^{12}$ and the six years outcome of the seventh patient ${ }^{13}$ have been reported previously.

\section{METHODS}

\section{Patient selection}

Seven patients with refractory TS were referred to the Maastricht University Medical Centre (MUMC) and selected for bilateral DBS of the medial thalamus between 2001 and 2008. One other patient underwent thalamic DBS during that period. This was a 20year-old institutionalized female patient with intractable TS and severe comorbidity, including life-threatening self-injurious behaviour. She underwent surgery for clinical urgency in 2006. Postoperative she experienced abrupt onset hypertonia, inconsistent 
and incongruent with organic disorders, bizarre movements and concomitant somatizations, suggesting a psychogenic nature. In 2009 she passed away in a nursing home. No follow-up data are available and the details have been described in a case report ${ }^{14}$.

Inclusion criteria for surgery were: 1) a primary diagnosis of TS according to the criteria of the Diagnostic and Statistical Manual of Mental Disorders criteria (fourth edition) (DSM-IV) ${ }^{15}$, 2) minimum age of 25 years, 3) a minimum score of 80 on the Diagnostic Confidence Index ${ }^{16}$, 4) a minimum score of 25 on the YGTSS ${ }^{17}$, 5) failure to respond to, or intolerable side effects of three-months trials of adequately dosed typical (e.g. haloperidol) or atypical (e.g. risperidone, olanzapine, quetiapine) antipsychotic medication or clonidine, 6) completed at least ten sessions of behavioural therapy. Exclusion criteria included: 1) tics not related to TS, 2) major psychiatric disorders, 3) current substance abuse or dependence (except for nicotine), 4) severe cognitive impairment, 5) structural abnormalities on brain Magnetic Resonance Imaging (MRI), 6) general contraindications for surgery or anaesthesia. All patients were evaluated by a multidisciplinary team, a collaboration of neurologists, neurosurgeons, psychiatrists and neuropsychologists with special interest in TS, in order to ensure appropriate selection. These results represent a systematic audit of routine outcome measurements and formal approval by a medical ethics committee was therefore not required. The shortterm results of all patients have been published previously ${ }^{12,13}$, and all patients gave written informed consent to report their results and to retrieve data from their medical records. To retrieve these long-term follow-up data all patients gave oral informed consent.

\section{Surgery}

Stereotactic bilateral DBS of the medial thalamus was performed under local anaesthesia and sedation in all seven patients. Target was the centromedian nucleus, substantia periventricularis, and nucleus ventro-oralis internus ( $\mathrm{Cm}-\mathrm{Spv}$-Voi) cross point of the thalamus, using the following standard coordinates; $x=5 \mathrm{~mm}$ lateral of the anterior commissure - posterior commissure (AC-PC) midline, $y=4 \mathrm{~mm}$ posterior to the mid-commissural point at the level of the AC-PC plane, $z=$ at the level of the AC-PC plane. Target coordinates were adapted according to the width of the third ventricle and the AC-PC length (Table 1). The trajectory with the best clinical outcome and no stimulation induced side effects was determined using extracellular single-unit microelectrode recordings (MER) and test stimulation. Full details of our neurosurgical procedure are published previously ${ }^{12}$. 
Thalamic Deep Brain Stimulation

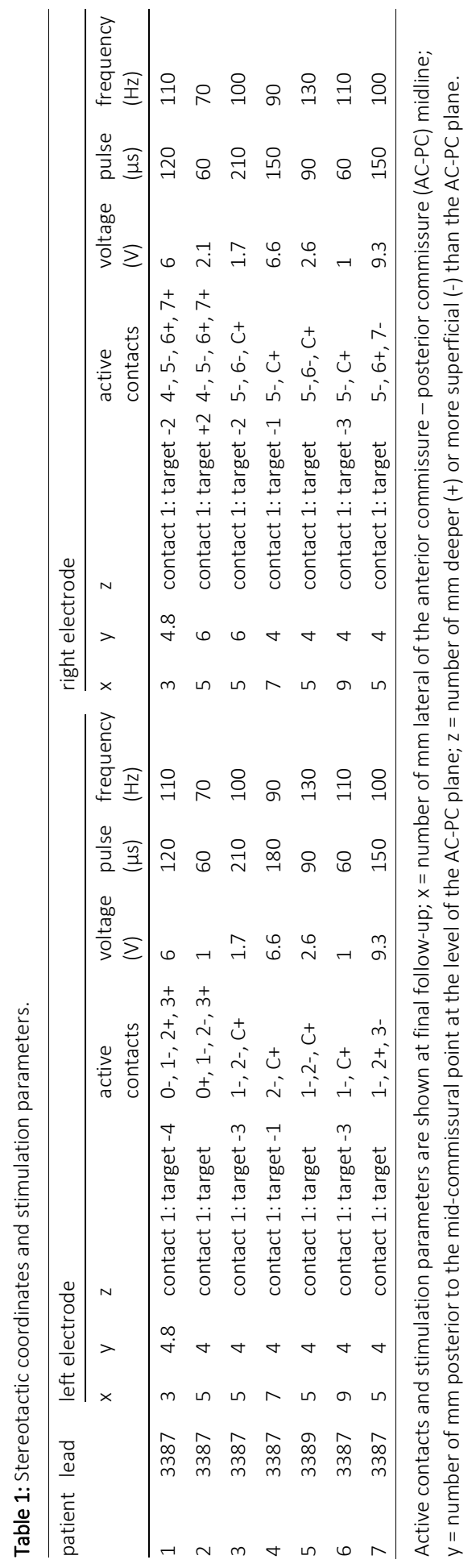




\section{Postoperative management}

Outpatient visits and programming sessions were performed as frequently as needed and wanted. Patients received a patient-programmer, which allowed them to change the stimulation voltage between individually assigned ranges. The initial goal was to evaluate all patients once a year, but due to non-compliance we were not able to collect data on a yearly basis. During visits to the outpatient clinic the effect on tics, side effects, complications and stimulations parameters were evaluated. At some moments, the YGTSS was completed by a neurosurgeon (LA) which provides an evaluation of number, frequency, intensity, complexity, and interference of motor and vocal tics. Total scores range from 0 to 50 with higher scores indicating higher severity ${ }^{17}$.

\section{RESULTS}

Patient 1 (male, 48 years at time of surgery)

This patient developed his first tics at the age of six. Motor tics consisted mainly of facial grimaces, eye blinking, and shoulders shrugs. Vocal tics were coughing and making uttering sounds. His tics were not responsive to medication or behavioural therapy. No comorbid disorders were present. In 2005 bilateral DBS of the thalamus was performed. After surgery, tics diminished and the YGTSS improved from 38 to 2 (94.7\%) at one-year follow-up (Table 2). However, he experienced many psychosocial stressors since he lost his job a few months after surgery. He suffered from several periods of minor depression during the following years. At 60 months of follow-up tic improvement was maintained with a score of 7 on the YGTSS (81.6\%). However, from 2009 he needed higher voltage stimulation to obtain the same effect on his tics. As a consequence, more frequent battery changes were needed, including a rechargeable battery. He was admitted to the hospital several times to obtain the most effective stimulation parameters without success. Side effects like a reduced level of energy, sleeping disorders and gaze disturbances became more severe, making stimulation at adequate parameters impossible. The active contacts and stimulation parameters at final followup are shown in Table 1. He was not satisfied with the effects of the stimulation anymore and bilateral DBS of the anterior GPi was carried out in 2012. The thalamic leads remained in situ but switched off. We were not able to reach a satisfying effect with pallidal stimulation on tics so far, even after rigorous adjusting of stimulation parameters. Finally, we decided to switch off the pallidal DBS and turn on the thalamic DBS again. No data, including the YGTSS, have been obtained during this period. 


\section{Patient 2 (male, 39 years at time of surgery)}

This patient developed tics around the age of six. He suffered from socially and physically debilitating motor and vocal tics, especially spitting and coprolalia. He also exhibited self-injurious behaviour like banging his forehead against a wall. He did not suffer from comorbidities. Medication and cognitive behavioural therapy were not effective. In 2005, he was selected for bilateral DBS of the thalamus. After surgery he developed a vertical gaze paralysis due to a small deep bleeding in the upper mesencephalon at the tip of the left electrode, which improved spontaneously ${ }^{18}$. The YGTSS improved from 44 to 19 (56.8\%) at 12 months of follow-up, and it improved even further at 24 months of follow-up (YGTSS of 11 (75\%)). Coprolalia and self-injurious behaviour disappeared after surgery. Although the vertical gaze palsy had resolved, he continued having visual disturbances and pressure behind his eyes during stimulation. He also experienced a reduction in his energy level. The YGTSS remained 22 (50\%) at 36 months of follow-up. However, due to these side effects and the burden of visiting the outpatient clinic, he eventually decided to switch the stimulator permanently off.

\section{Patient 3 (male, 40 years at time of surgery)}

This patient developed tics at the age of seven. He suffered from several motor tics like shoulder shrugs and neck extensions; however, most debilitating were his vocal tics, mainly coprolalia. He had a history of substance abuse, but no other comorbidities. In 2005 bilateral DBS of the thalamus was carried out. Postoperative he developed an infection of his infraclavicular pulse generator (staphylococcus aureus), which was successfully treated with six weeks of intravenous antibiotics. Tics progressively diminished after surgery and the YGTSS decreased from 45 to 12 (73.3\%) and further to $5(88.9 \%)$ at respectively 45 and 78 months of follow-up. This patient still experiences some side effects like reduced levels of energy and minor visual disturbances, especially with higher voltage stimulation. However, with the current stimulation parameters these side effects are acceptable and he is still satisfied with the effect of the DBS.

\section{Patient 4 (male, 35 years at time of surgery)}

This patient experienced his first tics at the age of seven. Motor tics included flexion with both arms and jumping and vocal tics included shouting, coprolalia and echolalia. Most debilitating were his vocal tics. He had a positive family history for TS and a history of depression and ADHD. In 2006 bilateral DBS of the thalamus was performed, with an improvement from 46 to $30(34.8 \%)$ on the YGTSS at 16 months of follow-up. From 2009 he started to suffer from more serious side effects like reduced levels of energy and visual disturbances, making daily activities impossible. Due to these side effects and the burden of the disease, he did not tolerate the same voltage stimulation as before 
anymore and the positive effects of stimulation began to decrease. In September 2012 the whole system had to be removed due to a persisting hardware infection after a pulse generator replacement, not treatable with antibiotics. Tics increased to the preoperative level with a score of 48 on the YGTSS. In December 2012 DBS of the anterior GPi was carried out. An improvement from 48 to 17 (64.5\%) was observed on the YGTSS after 12 months $^{19}$.

\section{Patient 5 (male, 40 years at time of surgery)}

This patient suffered from severe motor tics since the age of 12, most debilitating were forceful jerks of legs, arms and abdomen, facial grimaces and jumping. No comorbidities were present. In 2008 he was selected for bilateral DBS of the thalamus. His YGTSS showed a minor improvement from 40 to 29 (27.5\%) at 12 months after surgery. This patient was discontented with the results and went to Belgium for DBS of the GPe and was lost to follow-up.

\section{Patient 6 (male, 40 years at time of surgery)}

This patient developed tics at the age of six, during childhood motor tics decreased but vocal tics became more pronounced. Most debilitating were coprolalia, echolalia and uttering sounds. He had a history of substance abuse, but no other comorbidities. Bilateral DBS of the thalamus was carried out in 2008. Both motor and vocal tics diminished during stimulation, but almost all tics remained present to some extent. The YGTSS improved from 41 to 27 (34.1\%) at 26 months of follow-up. Postoperative, he experienced several symptoms like binge eating, lethargy, dysarthria, gait disturbances and apathy up to one year after surgery. These symptoms were not related to adjustments in stimulation settings and their interpretation was complicated by inconsistencies in his subjective report during the interviews and the complexity. A CTscan performed six months after surgery revealed cerebellar atrophy, not present at preoperative imaging. Due to all these other symptoms and the lack of effect we turned the stimulator off and as such he was lost to follow-up.

\section{Patient 7 (male, 45 years at time of surgery)}

This patient developed tics at the age of eight which gradually worsened around adolescence. His most debilitating symptoms consisted of forceful head movements, leading to cervical myelopathy, and screaming. Moreover, sexual obsessions and compulsions such as breaking glasses were troublesome. In 2001 he was selected for bilateral DBS of the thalamus, which resulted in an improvement from 43 to 26 on the YGTSS (39.5\%) at eight months of follow-up. During follow-up, major adjustments to the stimulation parameters were made with a progression of monopolar stimulation 
towards bilateral bipolar stimulation and an increase from 2.4 to $9.3 \mathrm{~V}$. However, this caused serious side effects including reduced levels of energy, gaze disturbances and alteration of sexual function, making stimulation at adequate parameters impossible. After three to six years higher scores on the YGTSS were observed $(44,35,39)$. Moreover, with lower voltage stimulation serious side effects remained. In 2010 a hardware defect in the left electrode was detected and, consequently, the whole DBS system was removed. Since a satisfying situation with thalamic DBS had not been reached in the last years, DBS of the anterior GPi was carried out in September 2010, at the age of 54. With pallidal DBS he experienced a large improvement on the YGTSS from 46 till $9(80.4 \%)$, which was maintained after 38 months of follow-up ${ }^{19}$.

Table 2: Yale Global Tic Severity Scale.

\begin{tabular}{|c|c|c|c|c|c|c|c|}
\hline & \multicolumn{7}{|c|}{ YGTSS total (motor/vocal) } \\
\hline & Baseline & $6-12 m$ & $12-24 \mathrm{~m}$ & $24-36 \mathrm{~m}$ & $36-48 \mathrm{~m}$ & $48-60 \mathrm{~m}$ & $60-78 \mathrm{~m}$ \\
\hline Patient 1 & $38(19 / 19)$ & $2(0 / 2)$ & - & $5(0 / 5)$ & $8(4 / 4)$ & $7(4 / 3)$ & ND \\
\hline Patient 2 & $44(25 / 19)$ & 19(19/0) & $11(10 / 1)$ & $22(16 / 6)$ & $22(16 / 6)$ & ND & ND \\
\hline Patient 3 & $45(20 / 25)$ & $19(13 / 6)$ & $22(18 / 4)$ & - & $12(12 / 0)$ & - & $5(5 / 0)$ \\
\hline Patient 4 & $46(21 / 25)$ & $34(15 / 19)$ & $30(15 / 15)$ & ND & ND & ND & ND \\
\hline Patient 5 & $40(22 / 18)$ & $29(14 / 15)$ & ND & ND & ND & ND & ND \\
\hline Patient 6 & $41(16 / 25)$ & $26(10 / 16)$ & - & $27(13 / 14)$ & ND & ND & ND \\
\hline Patient 7 & $43(20 / 23)$ & $26(16 / 10)$ & - & - & $44(23 / 21)$ & $35(19 / 16)$ & $39(21 / 18)$ \\
\hline
\end{tabular}

YGTSS: Yale Global Tic Severity Scale; m: months; ND: No Data (because stimulator removed or switched off).

\section{DISCUSSION}

These seven cases show that thalamic DBS in patients with severe TS can be effective in reducing tics at the short-term ${ }^{12}$. However, at the long-term in only one patient (patient 3) the stimulator is still active. In three patients (patient 1, 4 and 7) the target has been changed to the anterior GPi due to side effects (e.g. reduced level of energy, sleeping disorders, gaze disturbances and alteration of sexual function). In one of those patients (patient 1) a satisfying effect on tics during pallidal stimulation could not be reached and finally we switched off the pallidal DBS and turned on the thalamic DBS again. Three patients (patient 2, 5, and 6) were not satisfied with the results of the DBS and decided to switch off their stimulator. Two of them (patient 5 and 6) showed only minor improvement during stimulation, whereas the third patient (patient 2) had a good effect but suffered from the adverse effects. These results are partially in line with the results of Motlagh et al ${ }^{11}$, with an improvement of more than $50 \%$ in three out of eight cases versus three out of seven cases in our sample. However, they did not report on side effects over the course of time, which appeared to increase in our sample and were often responsible for changing the target or switching off the stimulator. Porta et 
al $^{9}$ reported a significant reduction in tic severity (pre-DBS $80.83 \pm 11.98$, post-DBS $22.11 \pm 14.19, p<0.001)$ at six years follow-up, but also difficulties like noncompliance and differences in the opinions between the patients and the medical team ${ }^{9,10}$. In their sample side effects have been reported only as minor and not being a cause of the dissatisfaction, which is in contrast with our results.

At long-term follow-up we also found differences in opinions between the medical team and the patients as to what a satisfactory outcome is. Whereas professionals mainly focus on tic reduction, a patient's wellbeing does not only depend on tic reduction ${ }^{10,20}$. In our group we noticed that patients with tic improvements between $9 \%$ and $34 \%$ generally were dissatisfied with the results and end their stimulation. We also observed that patients who responded well in terms of tic reduction $(>50 \%)$, but experienced increasing side effects were dissatisfied too. The YGTSS is a quantitative method to determine the effect on tics ${ }^{17}$, but it does not necessarily represent the patient's satisfaction with the treatment. Satisfaction may concern many factors, which can be different for the individual patients. Besides, there will be a response shift with respect to expectations and ambitions, which may explain changes in satisfaction over time. Therefore, qualitative research concerning the perception of satisfaction, expectations, and ambitions in TS patients undergoing DBS is highly needed ${ }^{20}$.

In the earlier years of DBS, professionals often mentioned the 'burden of normality' as an explanation for dissatisfaction after surgery ${ }^{21}$. On being rendered 'disease' free, the process of successful adjustment primarily depends on the patient's ability to discard the roles associated with the disease and his capacity to adjust to leading a normal life. For TS patients this may be especially difficult since they suffer from a lifelong disabling condition, and the tics have played a major role in the development of their personality, education and both social and professional life. In addition, DBS in TS improves the symptoms (tics), with a collateral improvement of comorbid behavioural disorders in only a minority of patients. In the early period after surgery the relief of tic improvement may distract from essential other premorbid problems and side effects. However, these problems and side effects may become more obvious after a longer time, and at that time more difficult to accept. In general, one easily can get used to 'success', but it is hard to accept 'losses'.

Saleh et al ${ }^{5}$ analysed all reported complications and adverse events after DBS for TS and other psychiatric diseases. Long-term morbidity was reported in $16.5 \%$ of cases, in $6.2 \%$ this was related to the surgery or hardware and in $10.2 \%$ to chronic stimulation 5,12 . Stimulation related side effects included; anxiety $(n=7)$, mood changes $(n=2)$, psychosis $(n=1)$, apathy $(n=13)$, and alteration of sexual function $(n=6)$. Notably, apathy was observed only in patients with thalamic DBS. The majority of reported side effects appeared to be transitory or resolved with adjustments of stimulation settings. This is not in line with our experience during the long-term follow-up. The side effects in our group did most often not respond to adjustments in stimulation settings. Moreover, the 
side effects, though based on self-report, became more pronounced with increasing treatment duration.

Thoughtful programming of the stimulator is very important to achieve an optimal clinical outcome ${ }^{11}$. Due to natural symptom fluctuations, variability in patient's responses to treatment and expectations, adjustment in stimulation settings is often a temporary solution ${ }^{9-11}$. In our experience TS patients tend to blame the stimulator for almost all negative symptoms and maybe professionals reinforce them to do so by adjusting the stimulation settings every time they report complaints. Given the experimental status of DBS in TS and their clinical responsibility to treat side effects, professionals might feel the pressure to intervene and as such maintain dissatisfaction. Intensive guidance, multidisciplinary, already early after surgery, is needed to differentiate between stimulation related changes and problems in coping with the postoperative situation. Standard postoperative psychoeducation for the patient and his system, either individual or in a group, will be helpful to shift the focus from the stimulator to the person and his environment and as such reduce blaming the stimulator and unnecessary adjustment of the stimulation settings.

The main limitation of this case series is that we report on observational data of a small sample and case descriptions from routine clinical practice with variable follow-up duration. Specialized Tourette clinics have reported only a handful of appropriate DBS candidates each year, rendering it nearly impossible to draw critical conclusions about the effects. However, the importance of these data for our daily practice cannot be underestimated. Thorough reporting on small groups and long-term follow-up data is essential, particularly in dealing with a complex disease like TS. Our experience highlights the need for more qualitative studies and the incorporation of additional outcome parameters other than reduction of tics and comorbid symptoms. To overcome some of these problems an International DBS Registry and Database for TS is being developed, with the idea that the statistical power necessary to refine and improve this procedure could only be achieved through the collection of a large worldwide community of cases ${ }^{22}$.

\section{CONCLUSION}

In our group of patients receiving thalamic DBS for TS there seems to be an increasing disbalance of therapeutic effects and side effects at long-term follow-up, often leading to either switching off the stimulator or new surgery with a different neuro-anatomic target. The reported cases reflect the strong heterogeneity of the disease and comorbidity, the still underrated impact of individual expectations and ambitions and the caution of the professionals, all interfering with effects and side effects and the overall success of DBS. 


\section{REFERENCES}

1. Bloch MH, Leckman JF. Clinical course of Tourette syndrome. Journal of psychosomatic research. 2009;67(6):497-501.

2. Leckman JF, Bloch MH, Scahill L, King RA. Tourette syndrome: the self under siege. Journal of child neurology. 2006;21(8):642-649.

3. Leckman JF. Tourette's syndrome. Lancet. 2002;360(9345):1577-1586.

4. Robertson MM. Tourette syndrome, associated conditions and the complexities of treatment. Brain. 2000;123 Pt 3:425-462.

5. Saleh C, Fontaine D. Deep brain stimulation for psychiatric diseases: what are the risks? Current Psychiatry Report. 2015;17(5):33.

6. Schrock LE, Mink JW, Woods DW, et al. Tourette syndrome deep brain stimulation: A review and updated recommendations. Movement disorders. 2014.

7. Baldermann JC, Schuller T, Huys D, et al. Deep Brain Stimulation for Tourette-Syndrome: A Systematic Review and Meta-Analysis. Brain Stimulation. 2016;9(2):296-304.

8. Servello D, Zekaj E, Saleh C, Zanaboni Dina C, Porta M. 16 years of Deep Brain Stimulation in Tourette's Syndrome: a critical review. Journal of neurosurgical sciences. 2016.

9. Porta M, Servello D, Zanaboni C, et al. Deep brain stimulation for treatment of refractory Tourette syndrome: long-term follow-up. Acta neurochirurgica. 2012;154(11):2029-2041.

10. Servello D, Sassi M, Brambilla A, Defendi S, Porta M. Long-term, post-deep brain stimulation management of a series of 36 patients affected with refractory gilles de la tourette syndrome. Neuromodulation. 2010;13(3):187-194.

11. Motlagh MG, Smith ME, Landeros-Weisenberger A, et al. Lessons Learned from Open-label Deep Brain Stimulation for Tourette Syndrome: Eight Cases over 7 Years. Tremor and other hyperkinetic movements. 2013;3.

12. Ackermans L, Duits A, van der Linden C, et al. Double-blind clinical trial of thalamic stimulation in patients with Tourette syndrome. Brain. 2011;134(Pt 3):832-844.

13. Ackermans $L$, Duits $A$, Temel $Y$, et al. Long-term outcome of thalamic deep brain stimulation in two patients with Tourette syndrome. Journal of neurology, neurosurgery, and psychiatry. 2010;81(10):1068-1072.

14. Duits A, Ackermans L, Cath D, Visser-Vandewalle V. Unfavourable outcome of deep brain stimulation in a Tourette patient with severe comorbidity. European child \& adolescent psychiatry. 2012;21(9):529-531.

15. Diagnostic and Statistical Manual of Mental Disorders: DSM-IV. Washington DC, American Psychiatric Association. 1994, ed 4.

16. Robertson MM, Banerjee S, Kurlan R, et al. The Tourette syndrome diagnostic confidence index: development and clinical associations. Neurology. 1999;53(9):2108-2112.

17. Leckman JF, Riddle MA, Hardin MT, et al. The Yale Global Tic Severity Scale: initial testing of a clinicianrated scale of tic severity. Journal of the American Academy of Child and Adolescent Psychiatry. 1989;28(4):566-573.

18. Ackermans L, Temel Y, Bauer NJ, Visser-Vandewalle V, Dutch-Flemish Tourette Surgery Study G. Vertical gaze palsy after thalamic stimulation for Tourette syndrome: case report. Neurosurgery. 2007;61(5):E1100; discussion E1100.

19. Smeets $A Y$, Duits $A A$, Plantinga BR, et al. Deep Brain Stimulation of the internal globus pallidus in refractory Tourette Syndrome. Clinical Neurology and Neurosurgery. 2016;142:54-59.

20. Dehning S, Leitner B, Schennach R, et al. Functional outcome and quality of life in Tourette's syndrome after deep brain stimulation of the posteroventrolateral globus pallidus internus: long-term follow-up. The world journal of biological psychiatry. 2014;15(1):66-75.

21. Wilson S, Bladin P, Saling M. The "burden of normality": concepts of adjustment after surgery for seizures. Journal of neurology, neurosurgery, and psychiatry. 2001;70(5):649-656

22. Deeb W, Rossi PJ, Porta M, et al. The International Deep Brain Stimulation Registry and Database for Gilles de la Tourette Syndrome: How Does It Work? Frontiers in Neuroscience. 2016;10:170. 


\section{Chapter 4}

\section{Deep Brain Stimulation of the internal globus}

pallidus in refractory Tourette Syndrome

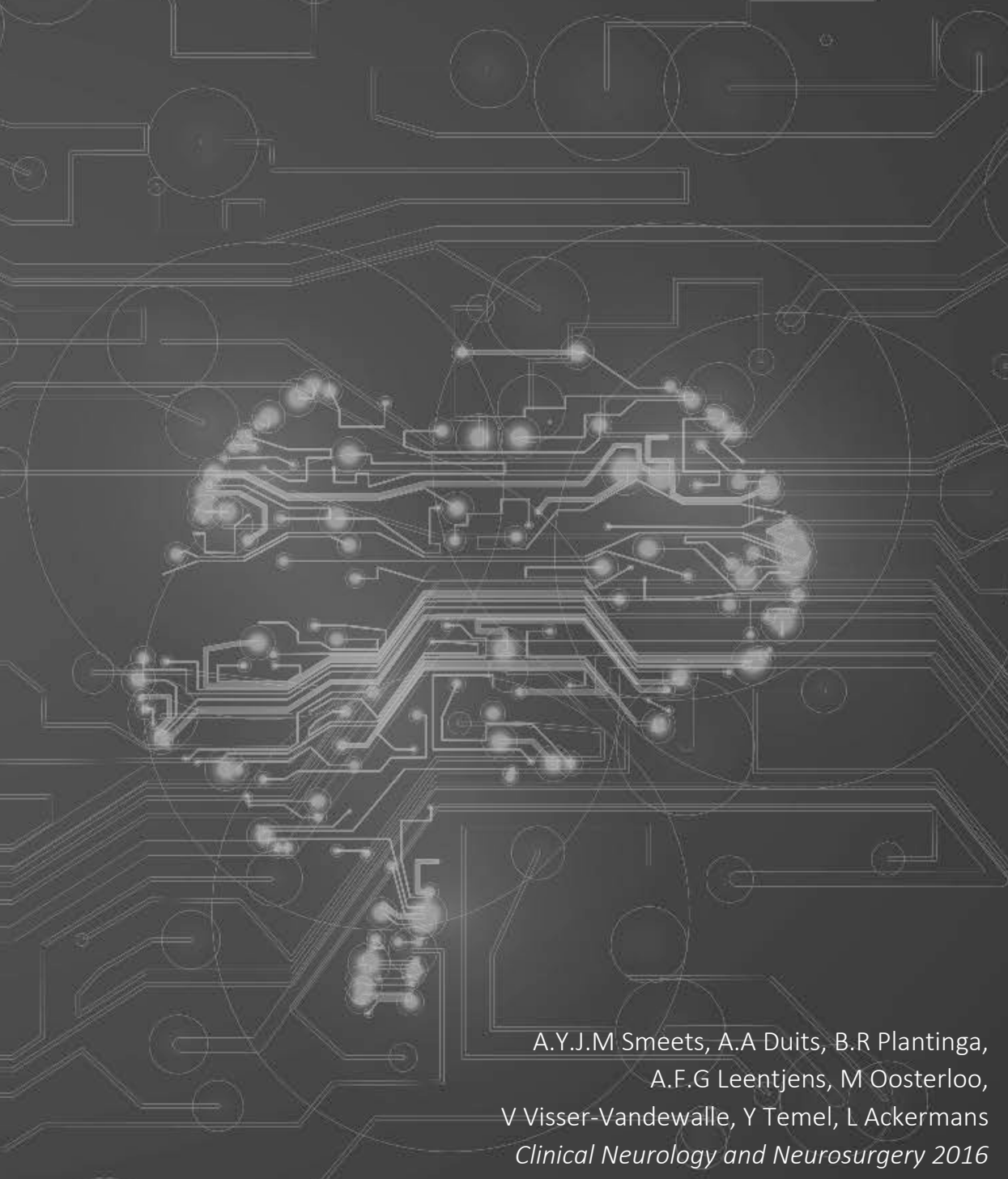




\section{ABSTRACT}

Objective: Deep Brain Stimulation in psychiatric disorders is becoming an increasingly performed surgery. At present, six different targets have been stimulated in Tourette Syndrome, including the internal globus pallidus. We describe the effects on tics and comorbid behavioural disorders of Deep Brain Stimulation of the anterior internal globus pallidus in five patients with refractory Tourette Syndrome.

Methods: This study was performed as an open label study with follow-up assessments between 12 and 38 months. Patients were evaluated twice, one month before surgery and at long-term follow-up. Primary outcome was tic severity, assessed by several scales. Secondary outcomes were comorbid behavioural disorders, mood and cognition. The final position of the active contacts of the implanted electrodes was investigated and side effects were reported.

Results: Three males and two females were included with a mean age of 41.6 years (SD 9.7). The total post-operative score on the Yale Global Tic Severity Scale was significantly lower than the pre-operative score $(42.2 \pm 4.8$ versus $12.8 \pm 3.8, \mathrm{P}=0.043)$. There was also a significant reduction on the modified Rush Video-Based Tic Rating Scale $(13.0 \pm 2.0$ versus $7.0 \pm 1.6, P=0.041)$ and in the total number of video-rated tics (259.6 \pm 107.3 versus $49.6 \pm 24.8, P=0.043$ ). No significant difference on the secondary outcomes was found, however, there was an improvement on an individual level for Obsessive-Compulsive behaviour. The final position of the active contacts was variable in our sample and no relationship between position and stimulation effects could be established.

Conclusion: Our study suggests that Deep Brain Stimulation of the anterior internal globus pallidus is effective in reducing tic severity, and possibly also ObsessiveCompulsive behaviour, in refractory Tourette patients without serious adverse events or side effects. 


\section{INTRODUCTION}

Tourette Syndrome (TS) is a complex childhood-onset neuropsychiatric disorder characterized by multiple motor tics plus one or more vocal tics ${ }^{1-3}$. More than half of patients with TS show evidence of psychiatric comorbidity, exhibiting symptoms of Attention Deficit and Hyperactivity Disorder (ADHD), Obsessive-Compulsive behaviour $(\mathrm{OCb})$, and anxiety or mood disorders ${ }^{2,3}$. TS is often self-limiting ${ }^{4}$, but in about $20 \%$ of patients tics continue in adult life and require chronic treatment ${ }^{1}$. Behavioural therapy and medication may provide relief, but some patients remain refractory or experience unbearable side effects ${ }^{2}$. For those patients, surgery might be the treatment of last resort.

Deep Brain Stimulation (DBS) of the medial thalamus was introduced in 1999 as a new approach for refractory TS patients ${ }^{5}$. At present, six different targets have been stimulated, including the internal globus pallidus (GPi), which is divided in a posterolateral part, the motor area (posterior GPi), and an anteromedial part, the limbic area (anterior GPi) ${ }^{6}$. Although the majority of studies have targeted the medial thalamus with positive effects on tics, the effects on comorbid behavioural disorders are indistinct and serious side effects, such as reduced levels of energy, visual disturbances, sleeping disorders, and erectile dysfunction, have been reported ${ }^{7,8}$. Recent open-label studies and small double-blind trials suggest that DBS of the anterior $\mathrm{GPi}$ is an effective and well-tolerated treatment for refractory TS ${ }^{9-13}$. Sachdev et al ${ }^{11}$ reported their long-term follow-up of 17 patients with DBS in the anteromedial GPi. Overall, they showed a significant tic reduction around $45 \%$ at one month of follow-up which was maintained up to four years. Side effects related to the stimulation were mostly temporary and attenuated with adjustment of stimulation parameters (e.g. anxiety, agitation, dizziness). Kefalopoulou et al ${ }^{12}$ performed a relative large randomized, double-blind, crossover trial of anterior GPi stimulation in 13 refractory TS patients. They a reported significant improvement on the Yale Global Tic Severity Scale (YGTSS) of $15.3 \%$ during the blinded off-stimulation versus on-stimulation, which further improved during the open label stimulation phase (40.1\%). Side effects were comparable to those described previously.

Based on these promising results and pathophysiological considerations we decided to perform anterior GPi stimulation in five patients with refractory TS. The effects on tics, associated behavioural disorders, mood and cognition are described in this paper. 


\section{METHODS}

\section{Patient selection}

Patients with refractory TS were referred to the Maastricht University Medical Centre (MUMC) and selected for bilateral DBS of the anterior GPi. Inclusion criteria for surgery were: 1) a primary diagnosis of TS according to the Diagnostic and Statistical Manual of Mental Disorders criteria (fourth edition) (DSM-IV) ${ }^{3}, 2$ ) a minimum score of 80 on the Diagnostic Confidence Index ${ }^{14}, 3$ ) a minimum age of 25 years, 4) a minimum score of 25 on the YGTSS ${ }^{15}$, 5) failure to respond to, or intolerable side effects of three-months trials of adequately dosed typical (e.g. haloperidol) and atypical (e.g. risperidone, olanzapine, quetiapine) antipsychotic medication or clonidine, 6) completed at least ten sessions of behavioural therapy (e.g. exposure in vivo or habit reversal). Exclusion criteria included: 1) tics not related to TS, 2) major psychiatric disorders (e.g. schizophrenia, bipolar disorder), 3) current substance abuse or dependence (except for nicotine), 4) severe cognitive impairment, 5) structural abnormalities on brain Magnetic Resonance Imaging (MRI), 6) general contraindications for surgery or anaesthesia. These criteria are in line with the European guidelines for TS and previously published research 6,7,16. All patients were seen by a neurosurgeon, psychiatrist and neuropsychologist, and patient selection was based on a multidisciplinary decision. These results represent a systematic audit of our outcomes and ethical approval was therefore not required. However, all patients gave written informed consent to report the results and to retrieve data from their medical records.

\section{Surgery}

The surgery was performed under local anaesthesia and sedation (lorazepam, remifentanil and clonidine), using a Leksell stereotactic frame. The following stereotactic coordinates were obtained: $12 \mathrm{~mm}$ lateral, 6-9 mm anterior, and 0-3 mm superior to the midcommissural point. Target coordinates were visualized and adapted on high field T2 W MRI images. A burr hole was placed in line with the planned trajectory and multiple-electrode extracellular single-unit micro-electrode recordings (MER) were performed. Recording took place from $10 \mathrm{~mm}$ above target to $4 \mathrm{~mm}$ beneath target in 0.5 to $1.0 \mathrm{~mm}$ steps. Subsequently, test stimulation was performed along the trajectories with the best MER recordings to assess stimulation-induced side effects. Finally, test electrodes were replaced by a final, quadripolar electrode (model 3389 or 3387, Medtronic Kerkrade, the Netherlands) with the second deepest contact (contact 1 ) at the level of the pallidal target that gave no side effects or only at highest stimulus intensity. Subsequently, the pulse generator (Activa PC, Medtronic) was implanted in a separate procedure under general anaesthesia. On the second postoperative day an MRI-scan was performed to detect asymptomatic haemorrhages and 
to evaluate the position of the electrodes. Stimulation parameters were adjusted after surgery. More details about the stereotactic neurosurgical procedure have been published previously by Ackermans et al ${ }^{7}$.

\section{Electrode localization}

The final position of the active contacts of the implanted electrodes was visualized. To this end, the coordinates of the active contacts were calculated in the post-operative images by manually defined coordinates of the most distal point of the electrode artefact, and of a proximal point in the centre of the electrode artefact. Next, the brains of the pre- and post-operative MRI images were extracted with FSL's brain extraction tool (BET) ${ }^{17}$ to rigidly fuse the post-operative images with the pre-operative images, using FSL's Linear Image Registration Tool (FLIRT) ${ }^{18}$. Based on this registration the postoperative active contact coordinates were converted to the pre-operative image space. Finally, ITK-SNAP segmentation software was used to visualize the final position of the active contacts on the pre-operative MRI scans ${ }^{19}$.

\section{Outcome measures}

Patients were evaluated twice, within one month before surgery and minimal twelve months after surgery on all primary and secondary outcomes. All evaluations were performed by the same examiners (AS, AD, LA).

The primary outcome was tic severity assessed by the YGTSS ${ }^{15}$, the modified Rush Video-Based Tic Rating Scale (mRVRS) ${ }^{20}$, and the number of video-rated tics in 10 minutes. The YGTSS provides an evaluation of number, frequency, intensity, complexity, and interference of motor and vocal tics. Total scores range from 0-50 with higher scores indicating higher severity. The mRVRS and the number of video-rated tics were based on five 2-minute video fragments in three different conditions: interview, sitting alone, and walking. The mRVRS scores tic frequency, severity of tics, and the number of body areas involved. Scores range from 0-25 with higher scores indicating higher severity. Secondary outcomes were comorbid behavioural disorders, mood and cognition. OCb was measured by the Yale-Brown Obsessive-Compulsive Scale (Y-BOCS) ${ }^{21}$ and ADHD by the Conner's Adult ADHD Rating Scale (CAARS) ${ }^{22}$. Depressive symptoms were assessed by the Dutch version of the Beck Depression Inventory 2th edition (BDI-II) ${ }^{23}$ and anxiety by the Beck Anxiety Inventory (BAI) ${ }^{24}$. A neuropsychological examination was performed to assess cognition. Given the known frontostriatal dysfunction in TS and results on cognition in the past, the examination focused on attention and executive functions ${ }^{7}$. The test battery included the digit symbol coding and digit span subtest of the Wechsler Adult Intelligence Scale-third edition (WAIS III) ${ }^{25}$, the Trail Making Test $(\mathrm{TMT})^{26}$, the Stroop Colour Word Test ${ }^{27}$, and Category ${ }^{28}$ and Letter fluency ${ }^{26}$. 
Adverse events, meaning complications related to the surgery or side effects related to the stimulation, were monitored carefully and frequently by interview on the outpatient clinic.

\section{Statistical analyses}

Pair-wise comparisons of the respective pre- and post-operative outcome variables were performed with Wilcoxon signed-rank tests and a P-value $<0.05$ was considered significant. All data were analysed using Statistical Package for the Social Sciences version 20.0 software (SPSS, Chicago, IL, USA).

\section{RESULTS}

\section{Study population}

In total five patients, three males and two females, were indicated for bilateral DBS of the anterior GPi between 2010 and 2013. Mean age of the group was 41.6 years (SD 9.7, range 35-57) and mean disease duration was 33.2 years (SD 8.9, range 23-46). Three patients were on medication for TS pre-operative (patient 2, 3 and 4). This medication was reduced by approximately $50 \%$ post-operatively in all patients. Followup duration ranged from 12 to 38 months (SD 13.7).

Patient 1: This divorced and unemployed male developed tics at the age of eight which gradually worsened around adolescence. Most debilitating symptoms were forceful head movements and screaming. Moreover, sexual obsessions and compulsions such as breaking glasses were troublesome. In 2002, at the age of 45, he was selected for bilateral DBS of the thalamus (centromedian nucleus, substantia periventricularis, and nucleus ventro-oralis internus (CM-Spv-Voi) cross point), which resulted in a tic reduction of $82 \%$ at 8 months of follow-up. During follow-up, major adjustments in stimulation parameters have been made with a progression of monopolar stimulation towards bilateral bipolar stimulation and an increase from 2.4 to $8.5 \mathrm{~V}$. However, this caused serious side effects like reduced levels of energy and gaze disturbances. At six years of follow-up tic reduction remained $78 \%$, but even with lower voltage stimulation serious side effects remained. In 2010 a hardware defect in the left electrode was detected and, consequently, the whole DBS system was removed. Since a satisfying result of the thalamic DBS had not been reached anymore the last years, DBS of the anterior GPi was carried out at the age of 54 in 2010. Follow-up duration was 38 months.

Patient 2: This unmarried and unemployed female, with a positive family history of tics and $A D H D$, suffered from tics since the age of five, becoming more severe around the 
age of 25. Motor tics were mainly facial grimaces and eye blinking. Vocal tics like grunting and severe coprolalia caused most impairment. She also suffered from selfmutilating behaviour (e.g. touching hot pans and sharp knives) and OCb (e.g. repeating other people, checking). In 2010, at the age of 29, she was selected for DBS of the anterior GPi. Follow-up duration was 34 months.

Patient 3: This unmarried and unemployed female suffered from tics since the age of seven, which waxed and waned during childhood. From the age of 18 tic severity increased. Motor tics such as head banging and jerks throughout her whole body were most disabling. Vocal tics included coprolalia and uttering sounds. Serious OCb like checking doors and counting made daily activities difficult. DBS of the anterior GPi was performed in 2011 at the age of 41. Follow-up duration was 29 months.

Patient 4: This unemployed and married male with one child, had tics since the age of seven. Most disabling were his vocal tics, including shouting, coprolalia and echolalia. But he also suffered from severe motor tics like jumping and jerks of the arms. In 2006, at the age of 35, DBS of the thalamus (CM-Spv-Voi cross point) was performed with an improvement in tics of $35 \%$ one year after surgery. Although the effect on tics remained stable, he was not satisfied with the results due to side effects like gaze disturbances and reduced levels of energy. He felt like a different person and was not able to perform his daily tasks. Many adjustments in stimulation settings were made without sustained satisfying results. In 2012 the pulse generator and the extra-cranial leads had to be removed due to a persisting hardware infection after an internal pulse generator replacement. Because of the disappointing results of thalamic DBS at long-term followup, we choose not to replace the thalamic leads. In 2012, at the age of 41, he was selected for DBS of the anterior GPi. Follow-up duration was 12 months.

Patient 5: This employed and married male with one child experienced facial tics since the age of 12. In his late twenties both motor and vocal tics became more severe and jerks throughout his whole body were the most disabling symptom. He also suffered from vocal tics like uttering sounds and coughing. Tic frequency was extremely high, mainly due to persisting bouts of combined tics. DBS of the anterior GPi was performed in 2013, at the age of 35. Follow-up duration was 12 months. 


\section{Final position of the implanted electrodes}

Details of the final electrode position and stimulation parameters are shown in Table 1 and Table 2. Moreover, the position of the active contacts is shown in Figure 1a-1f. In patient 1 , the left and right active contacts are located around $2 \mathrm{~mm}$ anterior in the external globus pallidus (GPe). In patient 2, both active contacts are located cranial in the anterior part of the GPi. In patient 3, the left active contact is located in the cranial part of the anterior GPi, however, the right active contact is situated in the posterior part of the anterior GPi. In patient 4, both active contacts are located in the anterior $\mathrm{GPi}$, stimulating both the cranial and caudal parts since three active contacts are used. In patient 5, the active contacts are located at the medial border of the anterior GPi with the internal capsule.

Table 1: Final electrode position.

\begin{tabular}{|c|c|c|c|c|c|c|}
\hline \multirow[b]{2}{*}{ Patient } & \multicolumn{6}{|c|}{ Left electrode } \\
\hline & Lead & $x$ & $y$ & $z$ & electrode & number of $M E R$ \\
\hline 1 & 3387 & 12 & 8 & contact 1 on target -1.0 & central & 5 \\
\hline 2 & 3387 & 12 & 9 & contact 1 on target & medial & 3 (except lat/pos) \\
\hline 3 & 3389 & 12 & 6.6 & contact 1 on target -3.0 & central & 3 (except ant/med) \\
\hline 4 & 3389 & 13.2 & 10.9 & contact 1 on target +1.5 & central & 3 (except ant/pos) \\
\hline \multirow[t]{2}{*}{5} & 3389 & 11.2 & 8.9 & contact 1 on target -0.5 & lateral & 3 (except ant/med) \\
\hline & \multicolumn{6}{|c|}{ Right electrode } \\
\hline Patient & Lead & $x$ & $y$ & $z$ & electrode & number of MER \\
\hline 1 & 3387 & 12 & 8 & contact 1 on target -1.0 & anterior & 5 \\
\hline 2 & 3387 & 12 & 11 & contact 1 on target & central & 2 (cen/med) \\
\hline 3 & 3389 & 12 & 6.6 & contact 1 on target -3.5 & lateral & 3 (except ant/med) \\
\hline 4 & 3389 & 14.4 & 9.6 & contact 1 on target +1.5 & anterior & 4 (except pos) \\
\hline 5 & 3389 & 11 & 8.9 & contact 1 on target -0.5 & central & 3 (except ant/med) \\
\hline
\end{tabular}

MER: microelectrode recordings; ant: anterior; lat: lateral; med: medial; pos: posterior; $x=$ number of mm lateral of the anterior commissure - posterior commissure (AC-PC) midline; $y=$ number of mm anterior to the mid-commissural point at the level of the AC-PC plane; $z=$ number of $\mathrm{mm}$ deeper $(+)$ or more superficial (-) than the AC-PC plane.

Table 2: Stimulation parameters.

\begin{tabular}{|c|c|c|c|c|c|c|c|c|c|c|c|c|c|}
\hline \multirow[t]{2}{*}{ Patient } & \multicolumn{5}{|c|}{ Left electrode } & \multicolumn{5}{|c|}{ Right electrode } & \multirow{2}{*}{$\begin{array}{l}\text { Voltage } \\
(V)\end{array}$} & \multirow{2}{*}{$\begin{array}{l}\text { Pulse } \\
(\mu s)\end{array}$} & \multirow{2}{*}{$\begin{array}{l}\text { Frequency } \\
(\mathrm{Hz})\end{array}$} \\
\hline & 0 & 1 & 2 & 3 & case & 4 & 5 & 6 & 7 & case & & & \\
\hline 1 & & & & - & + & & & & - & + & 3.2 & 360 & 130 \\
\hline 2 & & - & & & + & & - & & & + & 5.6 & 330 & 180 \\
\hline 3 & & & & - & + & & & & - & + & 4.7 & 330 & 100 \\
\hline 4 & - & - & & + & & - & - & & + & & 5.0 & 180 & 130 \\
\hline 5 & & & - & - & + & - & - & & & + & 2.2 & 330 & 130 \\
\hline
\end{tabular}

V: Volt; $\mu s$ : microseconds; Hz: Hertz. 


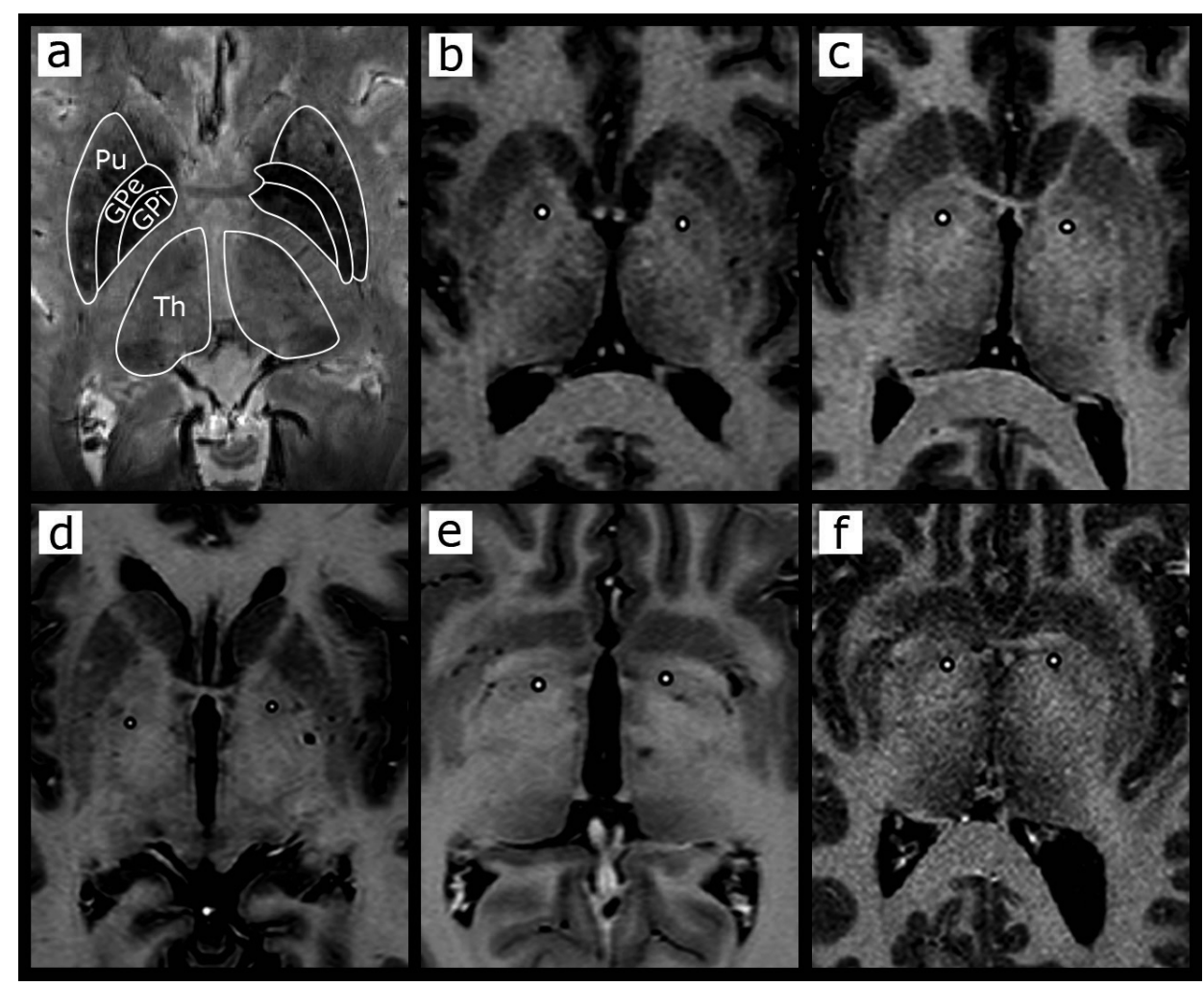

Figure 1: Final position of the active contacts of the implanted electrodes on the pre-operative inversion recovery $\mathrm{MRI}$-scans of the five patients, respectively.

(A): Anatomical overview around the internal globus pallidus. (B) Patient 1. (C) Patient 2. (D) Patient 3. (E) Patient 4. (F) Patient 5.

Abbreviations: Pu: Putamen; GPe: external globus pallidus; GPi: internal globus pallidus; Th: Thalamus. Pre-operative inversion recovery MR images were acquired with a $1.5 \mathrm{~T}$ MR scanner (patients 1, 2, and 5) and a 3 T MR scanner (patient 3 and 4) (Intera, Philips Medical Systems, Eindhoven, the Netherlands). The inversion time was $400 \mathrm{msec}$, and the echo time, repetition time, and resolution varied from 10-15 msec,

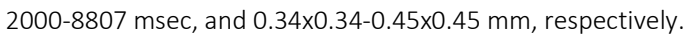

\section{Primary outcomes}

On a group level, the post-operative score on the YGTSS was significantly lower than the pre-operative score $(42.2 \pm 4.8$ versus $12.8 \pm 3.8, \mathrm{P}=0.043)$. There was also a significant reduction on the mRVRS $(13.0 \pm 2.0$ versus $7.0 \pm 1.6, \mathrm{P}=0.041)$ and on the total number of video-rated tics $(259.6 \pm 107.3$ versus $49.6 \pm 24.8, P=0.043)$. The YGTSS and the number of video-rated tics were divided into motor and vocal sub scores. For the videorated tics, improvements on both sub scores were comparable $(79.7 \%$ and $81.0 \%$, respectively). On the YGTSS the percentage of reduced motor scores was lower than that 
of the reduced vocal scores (64.8\% and $78.2 \%$, respectively). The evaluators were not blinded for the stimulation condition. Individual primary outcomes are shown in Table 3.

Table 3: Results on primary outcomes.

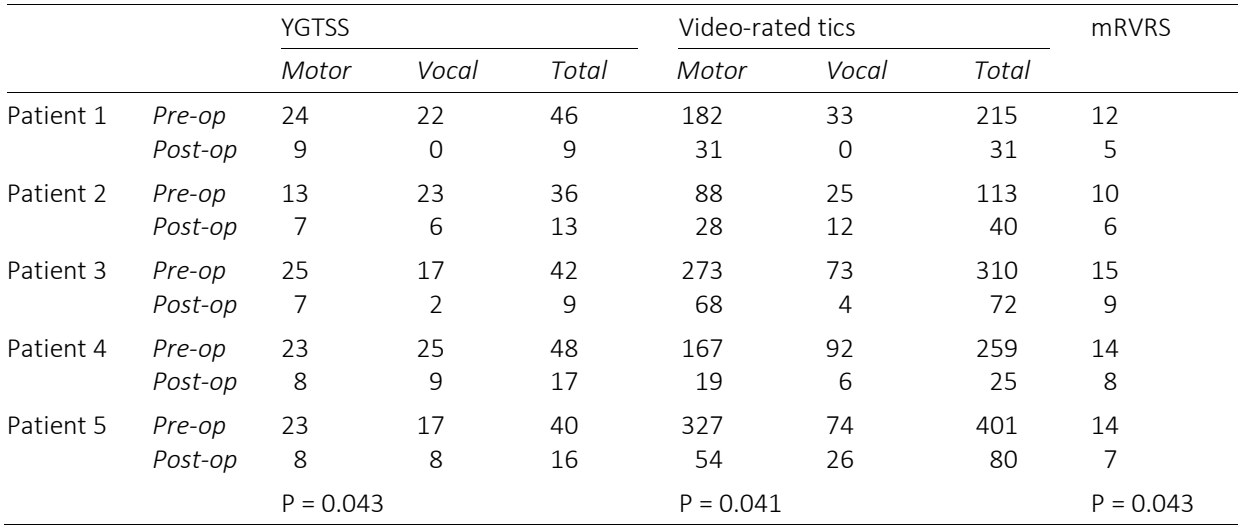

Pre-op: pre-operative; Post-op: post-operative; YGTSS: Yale Global Tic Severity Scale; mRVRS: modified Rush Video-Based Rating Scale.

\section{Secondary outcomes}

No significant differences were found between pre-operative and post-operative assessments of anxiety, depression and ADHD (Table 4). However, those patients (three of five) with clinically relevant symptoms of OCb prior to surgery (Y-BOCS > 16) showed improvement after surgery (12\%, 35\% and 100\%). Neuropsychological test scores revealed no significant differences between pre- and postoperative assessment (Table 5).

Table 4: Results on behavioural disorders and mood.

\begin{tabular}{|c|c|c|c|c|c|c|c|c|}
\hline & \multicolumn{2}{|l|}{ Y-BOCS } & \multicolumn{2}{|l|}{ CAARS } & \multicolumn{2}{|l|}{$\mathrm{BAI}$} & \multicolumn{2}{|l|}{$\mathrm{BDI}$} \\
\hline & Pre-op & Post-op & Pre-op & Post-op & Pre-op & Post-op & Pre-op & Post-op \\
\hline Patient 1 & 16 & 14 & 109 & 91 & 60 & 53 & 20 & 22 \\
\hline Patient 2 & 31 & 0 & 17 & 36 & 22 & 25 & 9 & 3 \\
\hline Patient 3 & 20 & 13 & 104 & 101 & 34 & 38 & 8 & 14 \\
\hline Patient 4 & 10 & 0 & 99 & 65 & 32 & 32 & 5 & 8 \\
\hline Patient 5 & 0 & 0 & MD & 35 & MD & 22 & MD & 1 \\
\hline Mean & $15.4(11.5)$ & $5.4(7.4)$ & $82.3(43.7)$ & 65.5 (29.8) & $37.0(16.2)$ & $34.0(12.3)$ & 10.5 (6.6) & $9.6(8.6)$ \\
\hline P-value & 0.068 & & 0.465 & & 1.000 & & 0.581 & \\
\hline
\end{tabular}

Mean (SD); Pre-op: pre-operative; Post-op: post-operative; Y-BOCS: Yale-Brown Obsessive-Compulsive Scale; CAARS: Conner's Adult ADHD Rating Scale; BAI: Beck Anxiety Inventory; BDI: Beck Depression Inventory; MD: Missing Data. 
Table 5: Results on cognition.

\begin{tabular}{llllllllll}
\hline & & $\begin{array}{l}\text { WAIS } \\
\text { digit } \\
\text { symbol } \\
\text { coding }\end{array}$ & $\begin{array}{l}\text { WAIS } \\
\text { digit } \\
\text { span }\end{array}$ & $\begin{array}{l}\text { TMT A } \\
\text { (sec) }\end{array}$ & $\begin{array}{l}\text { TMT B } \\
\text { (sec) }\end{array}$ & $\begin{array}{l}\text { Stroop } \\
\text { colour } \\
\text { word } \\
\text { (sec) }\end{array}$ & $\begin{array}{l}\text { Stroop } \\
\text { colour } \\
\text { word } \\
\text { (error) }\end{array}$ & $\begin{array}{l}\text { Category } \\
\text { fluency }\end{array}$ & $\begin{array}{l}\text { Letter } \\
\text { fluency }\end{array}$ \\
\hline Patient 1 & Pre-op & 42 & 11 & 40 & 101 & 80 & 0 & 37 & 32 \\
& Post-op & 45 & 15 & 40 & 110 & 102 & 0 & 35 & 25 \\
Patient 2 & Pre-op & 52 & 11 & 35 & 97 & 118 & 1 & 37 & 27 \\
& Post-op & 67 & 11 & 40 & 72 & 80 & 0 & 34 & 22 \\
Patient 3 & Pre-op & 99 & 23 & 12 & 30 & 50 & 0 & 59 & 72 \\
& Post-op & 83 & 24 & 13 & 59 & 62 & 0 & 47 & 58 \\
Patient 4 & Pre-op & 63 & 13 & 17 & 95 & 122 & 6 & 37 & 45 \\
& Post-op & 60 & 16 & 33 & MD & 101 & 3 & 36 & 39 \\
Patient 5 & Pre-op & 78 & 14 & 22 & 46 & 85 & 1 & 35 & 41 \\
& Post-op & 81 & 16 & 20 & 34 & 86 & 1 & 43 & 45 \\
\hline
\end{tabular}

Pre-op: pre-operative; Post-op: post-operative; sec: seconds; WAIS: Wechsler Adult Intelligence Scale; TMT: Trial Making Test.

\section{Adverse events}

Patient 1 developed a coagulase-negative staphylococcus infection of the abdominal pulse generator six months after surgery, which was successfully treated with intravenous antibiotics. Moreover, he experienced pain on the left side of his neck due to lead traction. Three patients experienced side effects related to the stimulation. Patient 1 and 3 reported a feeling of apathy leading to passive behaviour and not related to adjustments in stimulation parameters. Patient 4 experienced weight loss and stimulation-related agitation.

\section{DISCUSSION}

In this paper the effects of DBS of the anterior GPi were analysed in five refractory TS patients. Even though we performed an open label study with a small sample size, there was a significant beneficial group effect on tics. The improvement in YGTSS (70\%) is in line ${ }^{13}$ or even more ${ }^{11,12}$ than most previous studies targeting the same area report. Controlled data, although in a small sample, have even suggested that DBS of the anterior GPi could bring larger benefits than thalamic ${ }^{13}$ or posterior GPi ${ }^{10}$ stimulation. Although there were no significant effects for comorbid behavioural disorders, mood and cognition at the group level in our patients, those patients with pre-operative clinically relevant $\mathrm{OCb}$ showed post-operative improvement at an individual level. Effects of anterior GPi DBS on OCb have often not been described and are overall indistinct. Only Sachdev et al ${ }^{11}$ showed significant improvement in OCb during 
stimulation. So far, DBS in TS has not been associated with significant cognitive decline on a group level, providing support for its cognitive safety ${ }^{29}$.

Adverse events related to the surgery were minor and occurred in only one patient who developed an infection of his abdominal pulse generator. Stimulation induced side effects reported were apathy in two patients, not responsive to changes in stimulation settings. These patients were still able to perform all necessary daily tasks, with a higher threshold to initiate them. One patient suffered from agitation and weight loss, which was extensively investigated by several specialists, but no cause could be found. As such we believe the weight loss might be stimulation related, although adjustment of the parameters did not affect the weight. Apathy has been reported in other studies as a possible stimulation induced side effect ${ }^{12}$. Moreover, side effects like anxiety, agitation, and dizziness have been reported, which were not present in our group ${ }^{10-13,30}$.

The location of the active contacts differs between our five patients, ranging from the anterior part of the GPi to the GPe and the internal capsule. We could not establish a relation between the anatomical position of the active contacts and the effects of the stimulation. Larger sample sizes will be needed to clarify which anatomical structures relating to an anterior GPi trajectory provide optimal tic control. Our findings suggest that applying electrical stimulation to the region of the anterior parts of the GPe and GPi produces therapeutic effects on tics, and in patients with OCb possibly also on these symptoms.

This study has a number of limitations. We reported observational data from routine clinical practice with variable follow-up durations. As such no power calculation was performed and the statistical comparisons are based on five patients. There was no control group or control condition that would allow definite attribution of change in symptoms and side effects related to the implantation or stimulation. The paucity of available refractory TS patients, however, makes it difficult to perform highly needed randomized controlled trials with sufficient power.

\section{CONCLUSION}

Our study contributes to the sparse evidence in this field suggesting that anterior GPi DBS is an effective treatment in refractory TS ${ }^{10,13,30,31}$. We are cautious in over interpreting these data, but based on the present results, our experience with thalamic stimulation and other published evidence, we will continue targeting the anterior GPi as first choice in future refractory Tourette patients. 


\section{REFERENCES}

1. Bloch MH, Leckman JF. Clinical course of Tourette syndrome. Journal of psychosomatic research. 2009;67(6):497-501.

2. Robertson MM. Tourette syndrome, associated conditions and the complexities of treatment. Brain. 2000;123 Pt 3:425-462.

3. Diagnostic and Statistical Manual of Mental Disorders: DSM-IV. Washington DC, American Psychiatric Association. 1994, ed 4.

4. Jankovic J, Kurlan R. Tourette syndrome: evolving concepts. Movement disorders. 2011;26(6):1149-1156.

5. Vandewalle V, van der Linden C, Groenewegen HJ, Caemaert J. Stereotactic treatment of Gilles de la Tourette syndrome by high frequency stimulation of thalamus. Lancet. 1999;353(9154):724.

6. Schrock LE, Mink JW, Woods DW, et al. Tourette syndrome deep brain stimulation: A review and updated recommendations. Movement disorders. 2014.

7. Ackermans L, Duits A, van der Linden C, et al. Double-blind clinical trial of thalamic stimulation in patients with Tourette syndrome. Brain. 2011;134(Pt 3):832-844.

8. Servello D, Porta M, Sassi M, Brambilla A, Robertson MM. Deep brain stimulation in 18 patients with severe Gilles de la Tourette syndrome refractory to treatment: the surgery and stimulation. Journal of neurology, neurosurgery, and psychiatry. 2008;79(2):136-142.

9. Cannon E, Silburn P, Coyne T, O'Maley K, Crawford JD, Sachdev PS. Deep brain stimulation of anteromedial globus pallidus interna for severe Tourette's syndrome. The American journal of psychiatry. 2012;169(8):860-866

10. Martinez-Fernandez R, Zrinzo L, Aviles-Olmos I, et al. Deep brain stimulation for Gilles de la Tourette syndrome: a case series targeting subregions of the globus pallidus internus. Movement disorders. 2011;26(10):1922-1930.

11. Sachdev PS, Mohan A, Cannon E, et al. Deep brain stimulation of the antero-medial globus pallidus interna for Tourette syndrome. PloS one. 2014;9(8):e104926.

12. Wardell K, Kefalopoulou Z, Diczfalusy E, et al. Deep brain stimulation of the pallidum internum for Gilles de la Tourette syndrome: a patient-specific model-based simulation study of the electric field. Neuromodulation. 2015;18(2):90-96.

13. Welter ML, Mallet L, Houeto JL, et al. Internal pallidal and thalamic stimulation in patients with Tourette syndrome. Archives of neurology. 2008;65(7):952-957.

14. Robertson MM, Banerjee $S$, Kurlan R, et al. The Tourette syndrome diagnostic confidence index: development and clinical associations. Neurology. 1999;53(9):2108-2112.

15. Leckman JF, Riddle MA, Hardin MT, et al. The Yale Global Tic Severity Scale: initial testing of a clinicianrated scale of tic severity. Journal of the American Academy of Child and Adolescent Psychiatry. 1989;28(4):566-573.

16. Muller-Vahl KR, Cath DC, Cavanna AE, et al. European clinical guidelines for Tourette syndrome and other tic disorders. Part IV: deep brain stimulation. European child \& adolescent psychiatry. 2011;20(4):209-217.

17. Smith SM. Fast robust automated brain extraction. Human brain mapping. 2002;17(3):143-155.

18. Jenkinson M, Smith S. A global optimisation method for robust affine registration of brain images. Medical image analysis. 2001;5(2):143-156.

19. Yushkevich PA, Piven J, Cody H, Ho S, Gee JC, Gerig G. User-guided level set segmentation of anatomical structures with ITK-SNAP. Insight Jounral. 2005;1.

20. Goetz CG, Pappert EJ, Louis ED, Raman R, Leurgans S. Advantages of a modified scoring method for the Rush Video-Based Tic Rating Scale. Movement disorders. 1999;14(3):502-506.

21. Goodman WK, Price LH, Rasmussen SA, et al. The Yale-Brown Obsessive Compulsive Scale. I. Development, use, and reliability. Archives of general psychiatry. 1989;46(11):1006-1011. 
22. Conners CK, Wells KC, Parker JD, Sitarenios G, Diamond JM, Powell JW. A new self-report scale for assessment of adolescent psychopathology: factor structure, reliability, validity, and diagnostic sensitivity. Journal of abnormal child psychology. 1997;25(6):487-497.

23. Beck AT SR, Brown GK. Beck Depression Inventory-II. Manual. San Antonio, Texas, USA: Psychological Cooperation. 1996.

24. Beck AT, Epstein N, Brown G, Steer RA. An inventory for measuring clinical anxiety: psychometric properties. Journal of consulting and clinical psychology. 1988;56(6):893-897.

25. Wechsler D. Wechsler adult intelligence test. San Antonio, Tex, Psychological Corporation; 1997.

26. Spreen O. A compendium of neuropsychological tests: Administration, norms, and commentary. Oxford University Press; 1998.

27. Stroop JR. Studies of interference in serial verbal reactions. Journal of experimental psychology. $1935 ; 18(6): 643$.

28. Rosen WG. Verbal fluency in aging and dementia. Journal of clinical and experimental neuropsychology. 1980;2(2):135-146.

29. Duits A, Temel Y, Ackermans L, Visser-Vandewalle $V$. The cognitive safety of deep brain stimulation in refractory psychiatric disorders. Behavioural neurology. 2013;26(3):195-197.

30. Houeto JL, Karachi C, Mallet L, et al. Tourette's syndrome and deep brain stimulation. Journal of neurology, neurosurgery, and psychiatry. 2005;76(7):992-995.

31. Massano J, Sousa C, Foltynie T, Zrinzo L, Hariz M, Vaz R. Successful pallidal deep brain stimulation in 15year-old with Tourette syndrome: 2-year follow-up. Journal of neurology. 2013. 


\section{Chapter 5}

\section{Tonic and phasic changes in pallidal activity in Tourette Syndrome}

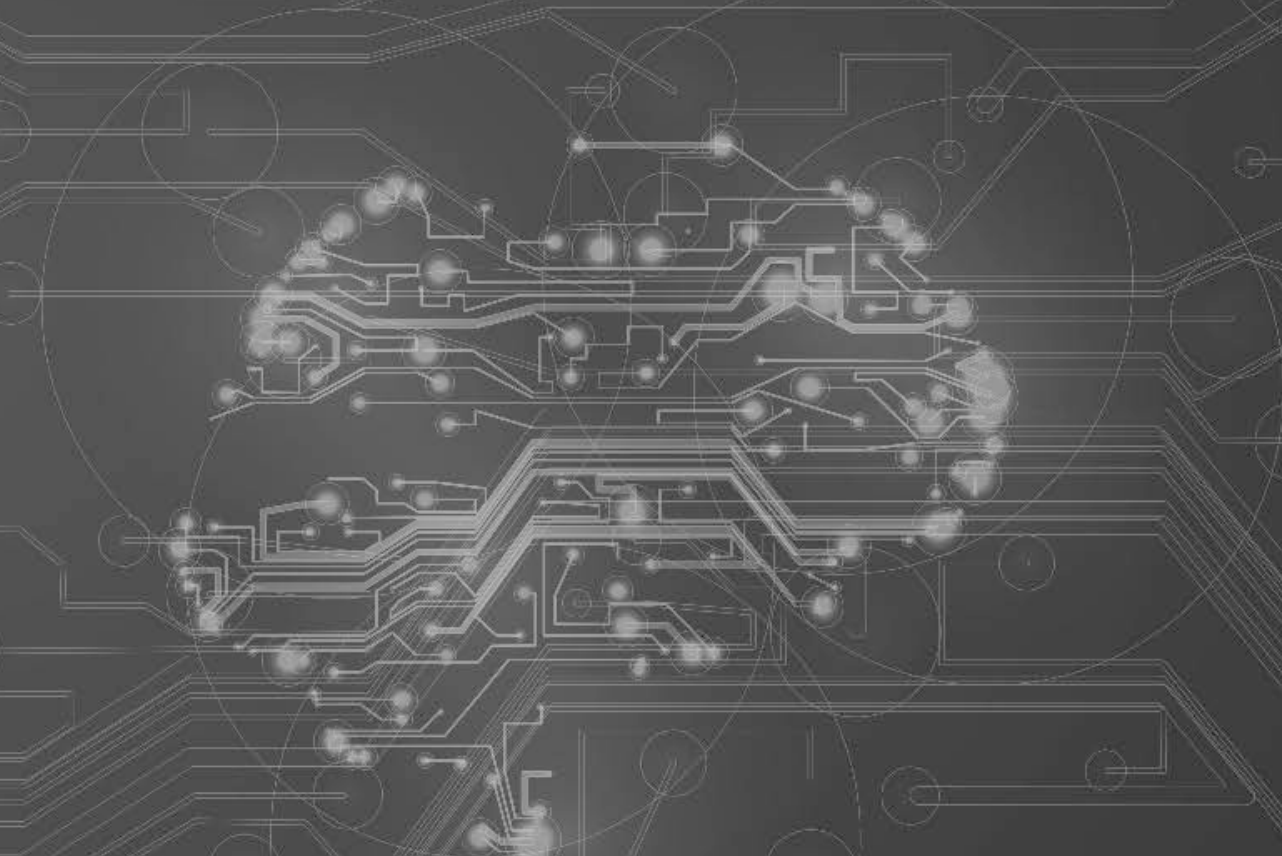

M Israelashvili, A.Y.J.M Smeets, M Bronfeld, D.H Zeef, A.F.G Leentjens, V van Kranen-Mastenbroek, M.L.F Janssen, Y Temel, L Ackermans, I Bar-Gad Movement disorders 2017 


\section{ABSTRACT}

Background: Tourette Syndrome is a hyperkinetic neurodevelopmental disorder characterized by tics.

Objective: Assess the neuronal changes in the limbic globus pallidus associated with Tourette syndrome.

Methods: Neurophysiological recordings were performed from the anterior (limbic) external globus pallidus and internal globus pallidus of eight awake patients during Deep Brain Stimulation electrode implantation surgeries.

Results: The baseline firing rate of the neurons was low in a state dependent manner in both segments of the globus pallidus. Tic dependent transient rate changes were found in the activity of individual neurons of both segments around the time of the tic. Neither oscillatory activity of individual neurons nor correlations in their interactions were observed.

Conclusions: The results demonstrate the involvement of the limbic pathway in the underlying pathophysiology of Tourette Syndrome and point to tonic and phasic modulations of basal-ganglia output as a key mechanism underlying the abnormal state of the disorder and the expression of individual tics, respectively. 


\section{INTRODUCTION}

Tourette syndrome (TS) is a childhood-onset neurodevelopmental disorder characterized by motor and vocal tics ${ }^{1}$. The underlying mechanism of TS is still largely unknown; however, multiple lines of evidence have linked it to malfunctions in the cortico-basal-ganglia-thalamocortical (CBGT) pathway ${ }^{2,3}$ and specifically to reduced inhibition within the striatum, the primary input nucleus of the basal ganglia (BG) $2,4,5$. The striatum controls the output of the BG through two pathways: the direct pathway to the output nucleus of the BG, the globus pallidus internus (GPi) and the indirect pathway to the GPi through the globus pallidus externus (GPe) ${ }^{6,7}$. These pathways are further subdivided into functional pathways that convey motor information primarily to the posterolateral GPi (posterior GPi) and limbic information to the anteromedial GPi (anterior GPi) ${ }^{8}$.

Currently, there is scant neurophysiological data from TS patients. Extracellular neuronal recordings performed during pallidotomy surgery revealed a decreased overall firing-rate in the posterior GPi of TS patients complemented by phasic firing-rate changes around the time of the tic ${ }^{9}$. Deep Brain Stimulation (DBS) targeting the anterior GPi in TS patients provides a rare opportunity to directly observe neuronal activity in the limbic domains of the BG. The current study focuses on linking the activity of GPe/GPi neurons in these domains with TS pathophysiology.

\section{METHODS}

\section{Patients}

The data were collected from eight patients, aged $19-55$ years $(37 \pm 14$, mean $\pm S D)$ who underwent surgery for the bilateral implantation of DBS electrodes in the anterior GPi, as treatment for TS (Table 1). Patients with refractory TS were referred to the Maastricht University Medical Centre (MUMC) and selected for bilateral DBS of the anterior GPi. Inclusion criteria for surgery were: 1) a primary diagnosis of TS according to the Diagnostic and Statistical Manual of Mental Disorders criteria (fourth and later fifth edition) ${ }^{10,11}$, 2) a minimum score of 80 on the Diagnostic Confidence Index ${ }^{12}$, 3) a minimum score of 25 on the Yale Global Tic Severity Scale (YGTSS) ${ }^{13}$, 4) failure to respond to, or intolerable side effects of three-months trials of adequately dosed typical (e.g. haloperidol), and atypical antipsychotic medication (e.g. risperidone, olanzapine, quetiapine) or clonidine, 5) completed at least ten sessions of behavioural therapy (e.g. exposure in vivo or habit reversal). Exclusion criteria included: 1) tics not related to TS, 2) major psychiatric disorders (e.g. schizophrenia, bipolar disorder), 3) current substance abuse or dependence (except for nicotine), 4) severe cognitive impairment, 5) structural abnormalities on brain Magnetic Resonance Imaging (MRI), 6) general contraindications 
for surgery or anaesthesia. These criteria are in line with the European guidelines for TS 14,15. Based on new insights and recommendations we have waived the previously suggested 25-years-old age limit since 2014, with the specification that a multidisciplinary team approach for screening is employed ${ }^{15}$. In this study, the neurophysiological results represent an overview of routine measurements performed during DBS surgery for TS and formal approval by a medical ethics committee was therefore not required. All patients gave written informed consent to report these results and to retrieve data from their medical records.

\section{Surgery}

Details about the neurosurgical procedure and the surgery outcomes are described in previous work ${ }^{16,17}$. The surgery was performed under local anaesthesia and sedation while the patients were awake. The following stereotactic coordinates were obtained: $12 \mathrm{~mm}$ lateral, 6-9 $\mathrm{mm}$ anterior, and 0-3 $\mathrm{mm}$ superior to the midcommissural point. Target coordinates were visualized and adapted on high field T2 weighted images. The angle of the track is adapted to T1 weighted images with contrast, to avoid blood vessels as well as the head of the nucleus caudatus and in order to enter the frontal lobes. Up to five micro-electrodes were used to record the neuronal activity along the trajectory for mapping purposes. Recording took place from $10 \mathrm{~mm}$ above target to $4 \mathrm{~mm}$ beneath target in $0.5-1.0 \mathrm{~mm}$ steps. The electrode signal was bandpass filtered $(160-5000 \mathrm{~Hz}$ ), and sampled at $20 / 25 \mathrm{kHz}$ (InoMed, V3.15). Movement during the neuronal recordings was monitored by simultaneous electromyogram (EMG) recordings, sampled at $2 \mathrm{kHz}$. EMG recordings were available only in three surgeries (patients $\mathrm{V}, \mathrm{K} \& \mathrm{O}$ ). The recordings were performed from the extensor digitorum communis and flexor carpi radialis (patient $\mathrm{V}$ ), from the masseter (patient's $\mathrm{K}$ and $\mathrm{O}$ ) and the deltoideus, rectus abdominus and femoralis (vastus medialis) on bilateral muscles (patient 0 ). In addition, a simultaneous video recording of the face was available in one surgery (patient K).

\section{Data pre-processing and analysis}

The anatomic location of the individual neurons was reconstructed based on the MRI scan, the overlaid trajectory and the trajectory history including border cells and areas of reduced neuronal activity (white matter). The signals were sorted offline (Offline Sorter, V2.8.8; Plexon) into multiple single-unit spike trains. All additional analyses were performed using custom-written MATLAB code (V2012B; MathWorks). Neurons were accepted for further analysis if they met the following criteria: 1) the recording was from a location within the GPe or the GPi, 2) the acquired cells' action potentials were of a consistent distinct shape that could be fully separated with a high degree of certainty from the spike waveforms of other neurons and the background noise, 3) the 
cells' inter-spike intervals were confirmed to have a refractory period, and 4) stable neuronal recording was available for at least 3 continues seconds (coefficient of variation (CV) or Fano factor (FF) < 4). Low-frequency bursting (LFB) neurons from the GPe were excluded from the database.

The firing rate, CV and FF of each neuron were calculated for the entire recording period of the neuron. As movement was not monitored for most patients, the time of the tics was unknown. Thus, the analyses were performed on a signal that was not free of the tics' effect on the overall baseline. FF was calculated using windows of $100 \mathrm{~ms}$.

Neuronal oscillations were assessed by estimating the power spectrum density (PSD) of the spike trains using Welch's method (Hamming window, 1s, 50\% overlap). A shuffling method was used to compensate for the distortion in the spectrum of spike trains which occurs due to the refractory period of the neurons. The mean of the PSD of each neuron was reduced from its PSD. Cross-correlation function between simultaneously recorded pairs of neurons was calculated using $1 \mathrm{~ms}$ bins and was smoothed with a Gaussian window $(S D=2 m s)$. The population correlations were assessed by calculating the Pearson's product-moment-correlation-coefficient (PCC) between the spike trains (bin size: 10ms) of simultaneously recorded neurons ( $>2 \mathrm{~s}$ overlap).

Changes in the neuronal firing rate around the tics were assessed by calculating the firing rate of each neuron during time windows of $1.5 \mathrm{~s}$ around the tics $(0.5 \mathrm{~s}$ pre- $1 \mathrm{~s}$ post tic onset time) and comparing this to the firing rate at all other times. The recordings used in our dataset were performed for mapping purposes, thus we only have a short recording period for each neuron containing very few tics (2.7 \pm 2.2 tics, mean $\pm S D)$. This precluded the use of methods with high temporal resolution, such as peri-tic time histogram, for the analysis of significant changes. We overcame the sparsity of the data by using broader windows thereby sacrificing temporal resolution for statistical significance. Tic onset times were calculated using threshold crossing of the EMG envelope (3/10 SD over the steady state mean value, for at least 3 consecutive bins). The EMG signals were processed offline and the envelope of the signal was estimated after low pass filtering $(8 \mathrm{~Hz}$, four-pole Butterworth filter) on the absolute values of the signal. EMG detected tics with a short time gap $(<1 s)$ from the previous tic onset were excluded. Frame-by-frame video analysis was used to characterize the motor tics in one patient (patient K). The video detections were then aligned to the EMG tic-detection and EMG detected tics that were not accompanied (preceded by<250ms) by a video detected tic (of type: eye blink) were excluded from the analysis. The significance level of the ticrelated changes was determined by calculating the distribution of a dataset containing 1000 sequences of sham tics for each neuron. Each sham tic sequence included random times which excluded the times of real tics. The number of sham tics within each sequence was set to the number of actual tics that occurred during the recording of each neuron. 


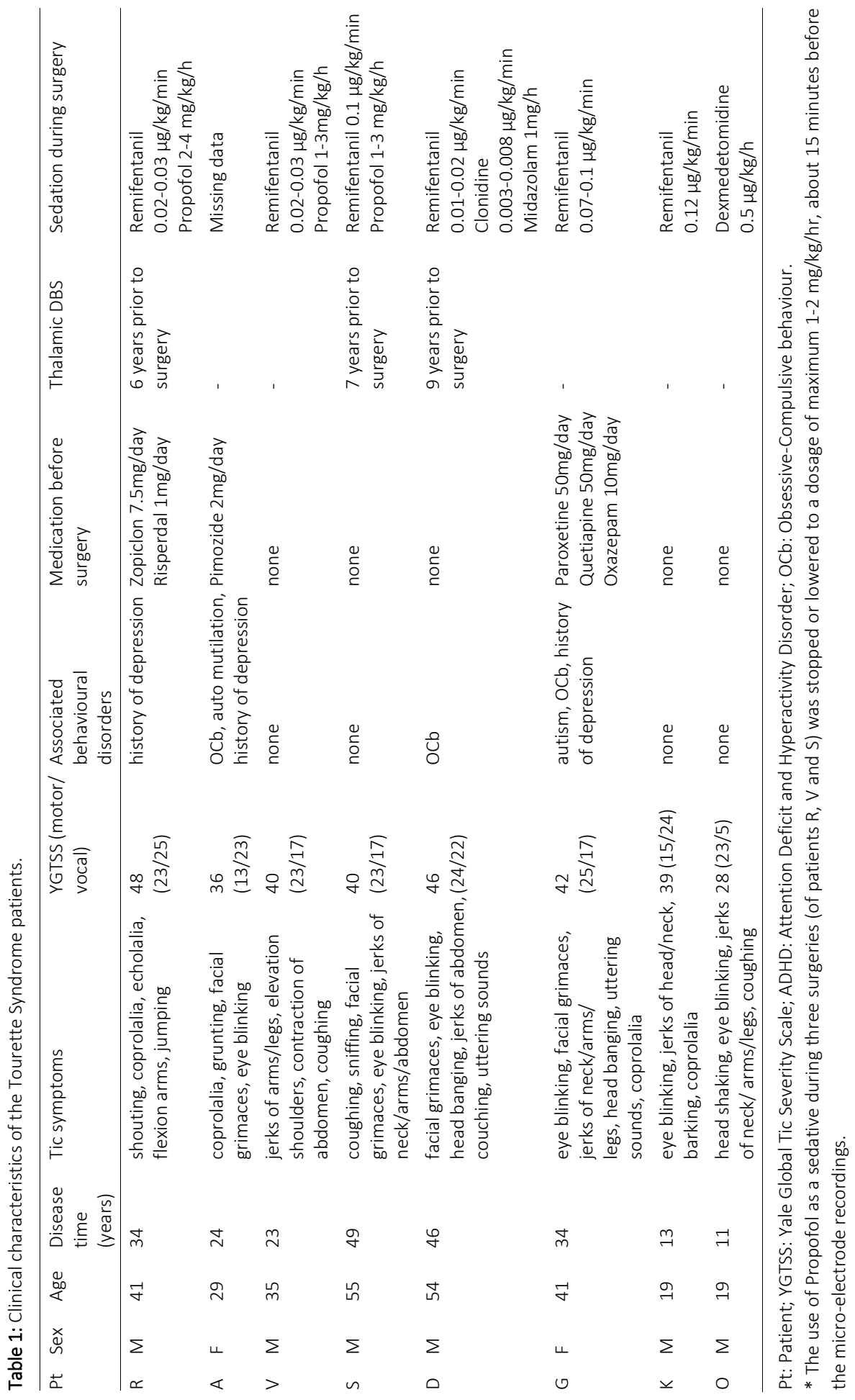




\section{RESULTS}

Firing rate and pattern

A total of 235 single neurons were recorded from eight awake TS patients during neurophysiological mapping for placement of DBS electrodes in the anterior GPi. Clinical characteristics of the patients are shown in Table 1. The neurons were identified as GPe $(n=77)$ or GPi ( $n=158)$ neurons based on their location and trajectory history including border cells and low background activity between the GPe and GPi (Figure 1A).

The firing rates were $48.25 \pm 3.4$ spikes/s and $50.9 \pm 2.6$ spikes/s (mean $\pm S E M$ ) for GPe and $\mathrm{GPi}$, neurons respectively. The firing rate distributions were positively skewed indicating that the firing rate of most neurons was even lower, with a small subpopulation of high frequency discharge neurons. While the firing rates were similar between GPe and GPi neurons, the firing patterns of the neurons in each nucleus were different. Both the CV and the FF were higher in GPe neurons, in line with their typical pauses, as compared to GPi neurons which presented Poisson-like values of CV and FF ( 1) (Figure 1B). Different pathologies of the BG, most notably Parkinson's disease (PD), are associated with enhanced oscillatory activity. We found no significant oscillations in the spike trains of neither GPe nor GPi neurons using power spectral estimation methods. 
A

i)

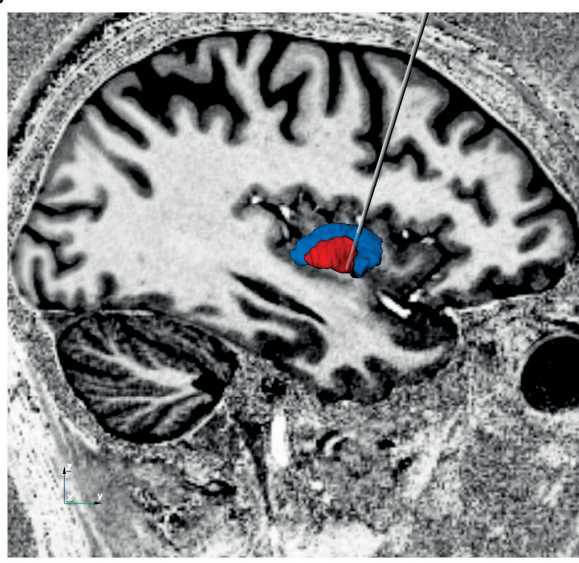

ii)

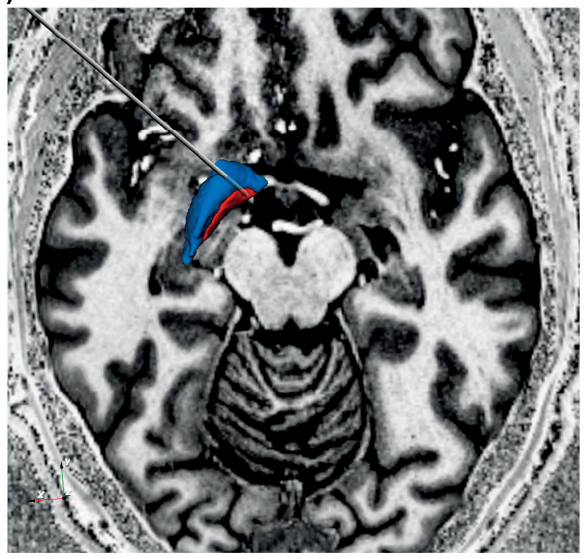

B

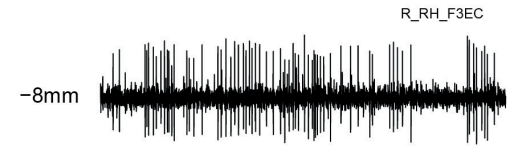

R_RH_F6EL

$-5 \mathrm{~mm}$

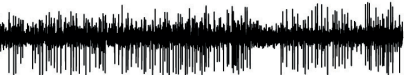

$\mathrm{GPe}$

GPi

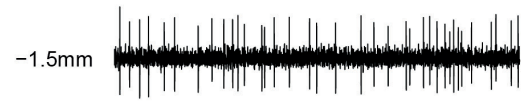

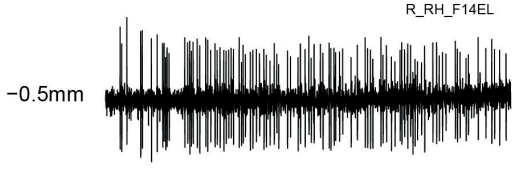

R_RH_F15EA

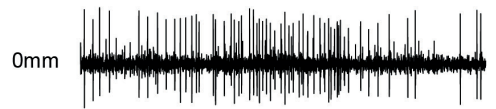

R_RH_F18EM

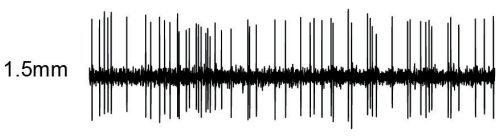

R_RH_F20EL

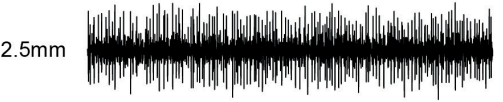

Figure 1A: Recording track.

(A) An example of the recording electrode track overlaid on (i) sagittal and (ii) axial MRI for purposes of illustration. Blue - GPe, red - GPi. Abbreviations: GPe: external globus pallidus; GPi: internal globus pallidus. (B) Examples of neurons recorded from several locations along one track. The electrode depth is shown in relation to the location of the stimulation electrode implantation site $(0 \mathrm{~mm})$. 
A

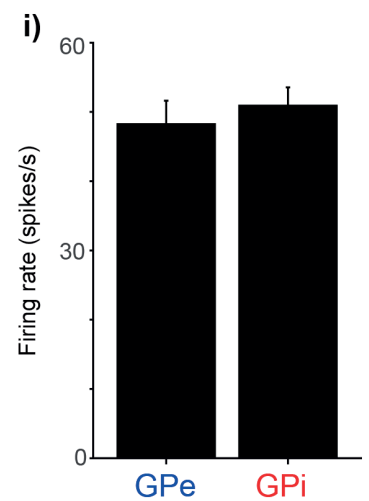

B

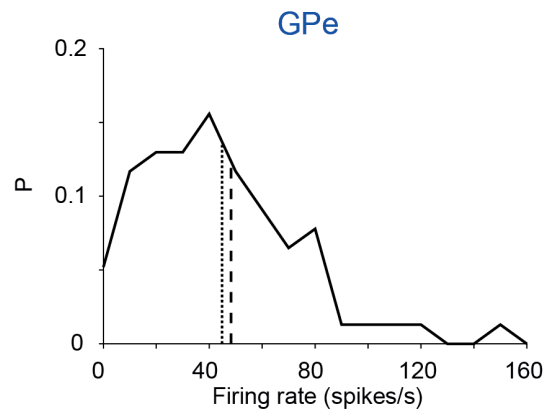

ii)

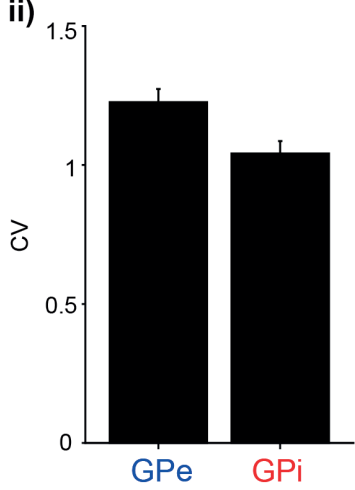

ii)

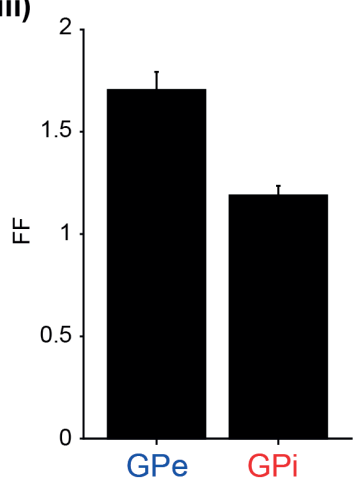

Figure 1B: Firing characteristics of globus pallidus neurons.

(A) Mean baseline firing rate (i), CV (ii) and FF (iii) of GPe and GPi neurons. Abbreviations: CV: coefficient of variation; FF: Fano factor; GPe: external globus pallidus; GPi: internal globus pallidus.

(B) Firing rate distributions of GPe and GPi neurons (bin size $=10$ spikes/s). The mean (dashed vertical line) and median (dotted vertical line) of each distribution are overlaid.

\section{Neuronal interactions}

Neuronal activity in the GPe/GPi of the normal non-human primate is uncorrelated ${ }^{18,19}$. However, positive correlations between these neurons appear in PD patients ${ }^{20,21}$ and in the primate model of the disorder ${ }^{19}$. To examine whether changes in firing synchrony play a role in the pathophysiology of tics, we examined the correlation in spike firing between simultaneously recorded neurons. Cross-correlation functions were calculated between the spike trains of 63 pairs (GPe:18, GPi:45) of simultaneously recorded single neurons. The activity of the neurons was found to be uncorrelated as none of the crosscorrelation functions had significant peaks (Figure 2A). We calculated the Pearson's product-moment-correlation-coefficient (PCC) between the spike trains of each pair of neurons. The average correlation coefficient for the whole population of pairs was zero with narrow margins $(0 \pm 0.03$, mean $\pm S D)$ (Figure $2 \mathrm{~B})$. Coherence was used to study the degree of neuronal interaction as a function of frequency. The mean coherence 
between all simultaneously recorded pairs was flat with no significant peak at any frequency (Figure $2 \mathrm{C}$ ).

A
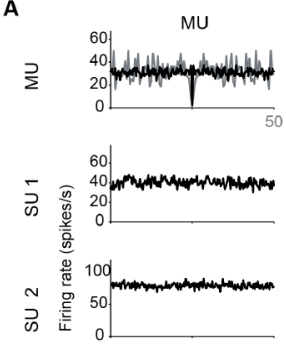

$\stackrel{n}{\infty}$

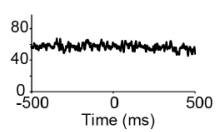

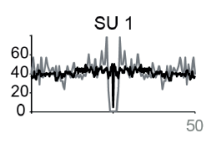
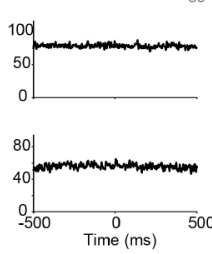

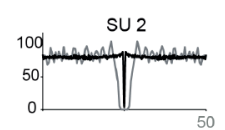

R_RH_F18EA,M,C,L (A) An example of the cross-correlation between simultaneously recorded neurons in the internal globus pallidus. Grey line, auto-correlation at a smaller time window of $50 \mathrm{~ms}$ (not smoothed). Abbreviations: $\mathrm{MU}$ : multiunit; SU: single unit.
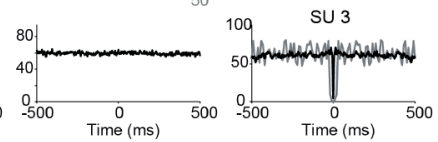

B

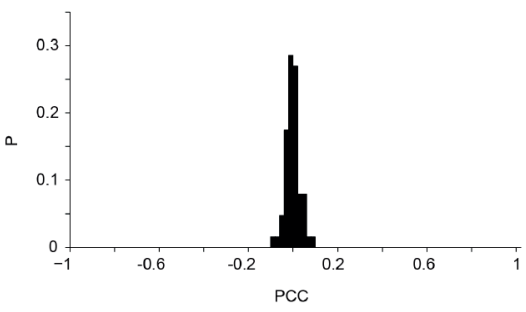

C

$\mathrm{GPe}$

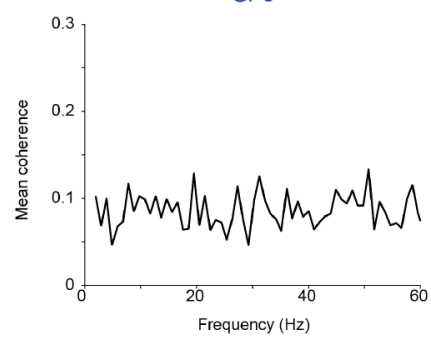

GPi

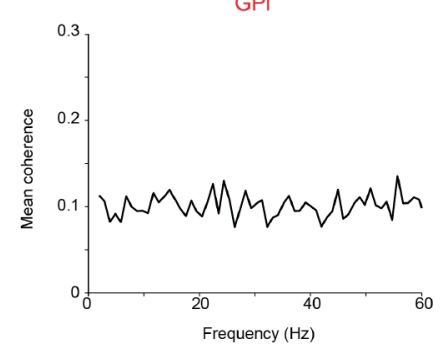

(B) Probability distribution of the Pearson's productmoment correlation coefficient (PCC) between the spike trains of all the simultaneously recorded pairs of neurons (bin=0.02).

(C) Mean coherence in the GPe and GPi.

Abbreviations: GPe: external globus pallidus; GPi: internal globus pallidus.

Figure 2: No correlations in the globus pallidus.

\section{Tic-related changes in neuronal activity}

The expression of tics was associated with altered neuronal activity throughout the globus pallidus (Figure $3 \mathrm{~A}$ ). Tic times were identified using EMG recordings from three patients (patients $\mathrm{V}, \mathrm{O}$ and $\mathrm{K}$; patient $\mathrm{K}$ had in addition a video recording). The mean firing rate of each neuron during 1.5 seconds $(-0.5 s \rightarrow+1 s)$ around tics onset was compared to the mean firing rate of the neuron during the rest of the time (using $100 \mathrm{~ms}$ time windows). A large fraction $(44.1 \%, 15 / 34)$ of GPe neurons significantly changed their firing rate around the time of the tic (two-sample t-test; 10/15 with 
$\mathrm{p}<0.01$, and 5/15 with $\mathrm{p}<0.05)$. Similarly, $41.7 \%$ (15/36) of GPi neurons significantly changed their firing rate around the time of the tic $(6 / 15$ with $p<0.01$, and $9 / 15$ with $\mathrm{p}<0.05)$ (Figure $3 \mathrm{~A}-\mathrm{B})$. In both nuclei, half of the responding neurons $(8 / 15 \mathrm{GPe}, 7 / 15$ GPi) displayed a decrease in activity around the time of the tic. The fraction of neurons displaying tic-related activity was significantly higher than expected by the distribution of changes around random time events ( $z$-score, GPe P<0.05; GPi P<0.01). In contrast to the significant firing rate changes of individual neurons, the mean response of GPe as well as of GPi population to the tics was not significantly different from the baseline activity (paired-sample t-test, $\mathrm{P}>0.05$ ) (Figure 3B-C, bar plot). Thus, despite phasic ticrelated changes in the firing rate of individual neurons, the mean firing rate of both nuclei did not phasically change during tic occurrence.

A

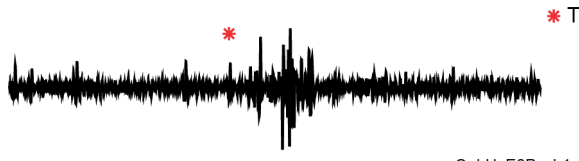

O_LH_E8P_ch1

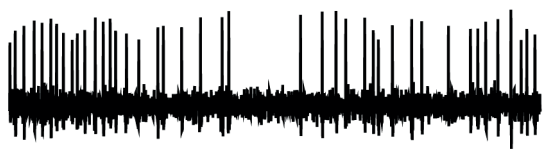

$0.1 \mathrm{~s}$

B

C
* Tic(A) An example of simultaneously recorded EMG (top) and GPe neuron (bottom) around the time of a tic.

Abbreviations: EMG: electromyogram; GPe: external globus pallidus.
$\mathrm{GPe}$
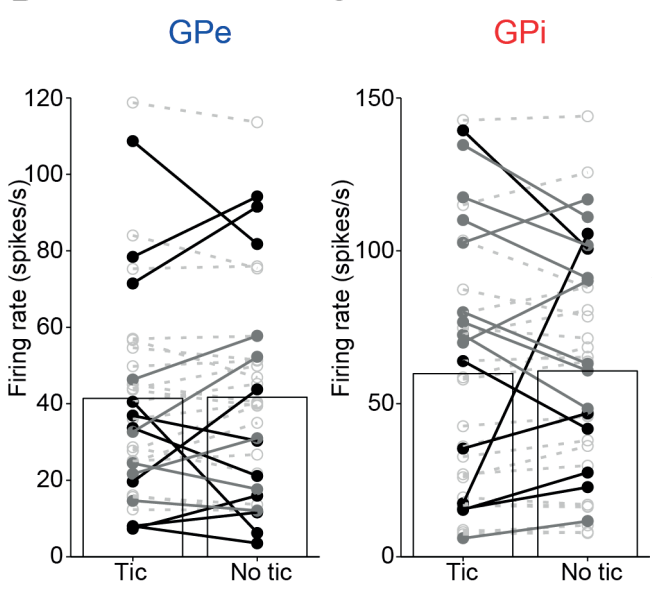

$p<0.01$

- $p<0.05$

Figure 3: Tic-related changes in firing rate.
(B-C) Mean firing rate around tic time $(0.5 \mathrm{~s}$ pre tic onset -> 1s post tic onset) for GPe (B) and GPi (C) neurons (calculated on data from three patients). Each dot represents one neuron. Coloured dots represent neurons that displayed significant firing rate changes around the time of the tics. The bar plot represents the mean firing rate of all neurons at each period (tics vs. no tics).

Abbreviations: GPe: external globus pallidus; GPi: internal globus pallidus. 


\section{DISCUSSION}

In this study, we examined neuronal activity in the anterior (limbic) GPe and GPi in TS patients. The baseline firing rate was low and the firing rate distribution of the neurons was skewed. In addition to the tonic reduction in the firing rates, a large fraction of the neurons displayed phasic tic dependent changes. These changes included transient increases and decreases in activity in both segments of the globus pallidus. In contrast to other BG related disorders, oscillatory activity of individual neurons as well as abnormal synchronization between neurons were not observed.

No studies of the limbic (anterior) GPi or any part of the GPe tonic activity during TS have been previously conducted. Tonic activity changes in the motor (posterior) GPi during TS have been partially addressed in previous studies of anesthetized ${ }^{22}$ and awake ${ }^{23}$ patients undergoing a mapping procedure. Our results are in line with both studies which found a rate decrease in the GPi compared to rates recorded in PD patients ${ }^{23}$ or normal non-human primates ${ }^{19}$. Interestingly, the reduced firing rates that we observed in the GPe are comparable to those previously seen in awake patients suffering from other hyperkinetic disorders such as dystonia ${ }^{22,24-26}$ and hemiballismus ${ }^{27}$.

Functional models of hyperkinetic movement disorders have tended to focus solely on the existence of abnormalities in the motor circuits of the BG. Here we found changes in the activity of the anterior (limbic) domains of both segments of the globus pallidus resembling those seen in the motor domain ${ }^{9}$. This points to a clear functional interaction between the motor and limbic circuits within the CBGT loop which is consistent with the symptomatology of TS that links limbic, e.g. psychiatric comorbidities, and motor, e.g. tics, properties ${ }^{28,29}$.

Hyperkinetic disorders in general, and TS in particular, are hypothesized to be associated with enhanced activity of the direct pathway and reduced activity of the indirect pathway which together leads to over-activation of the cortex ${ }^{6,7}$. In line with this assumption, imaging studies have found abnormal activity in the globus pallidus and enhanced excitation of the direct pathway ${ }^{30-32}$. Our results support this assumption in that the GPi neurons displayed reduced activity. However, the GPe baseline, tonic, activity was also reduced, contradicting the current perception of the indirect pathway involvement in hyperkinetic disorders ${ }^{6,7}$. Interestingly, this co-modulation of both segments of the globus pallidus is not unique to TS, but has also been observed in other hyperkinetic disorders such as dystonia ${ }^{25,26}$ and hemiballismus ${ }^{27}$. The reduced activity in both segments can be explained by later theoretical models ${ }^{33,34}$ and experimental data from animal models ${ }^{35,36}$ that point to striatal disinhibition as a key factor driving the appearance of tics. This disinhibition, affecting striatal projection neurons contributing to both the direct and indirect pathways, is thus expected to exercise increased inhibition on both the GPe and GPi driving their firing rates down, in line with our findings. The mixture of phasic responses around the time of the tic indicates that 
the neurons within each nucleus form a heterogenic network, in line with previous findings in animal models of the disorder ${ }^{35,36}$.

\section{CONCLUSION}

Our results support and extend existing theoretical, clinical and experimental data suggesting tonic and phasic changes in the neuronal activity throughout the BG during TS. Moreover, our results are unique in demonstrating that these changes affect not only motor pathways but also limbic pathways reflecting the complexity of the disorder. The departure of our findings from the classic perception of hyperkinetic disorders thus calls for the development of a more comprehensive model of BG functionality linking the activity in different domains of the BG in general and during hyperkinetic disorders in particular. 


\section{REFERENCES}

1. Bloch MH, Leckman JF. Clinical course of Tourette syndrome. Journal of psychosomatic research. 2009;67(6):497-501.

2. Kalanithi PS, Zheng W, Kataoka Y, et al. Altered parvalbumin-positive neuron distribution in basal ganglia of individuals with Tourette syndrome. Proceedings of the National Academy of Sciences of the United States of America. 2005;102(37):13307-13312.

3. Singer HS, Minzer K. Neurobiology of Tourette's syndrome: concepts of neuroanatomic localization and neurochemical abnormalities. Brain \& development. 2003;25 Suppl 1:S70-84.

4. Kataoka Y, Kalanithi PS, Grantz H, et al. Decreased number of parvalbumin and cholinergic interneurons in the striatum of individuals with Tourette syndrome. The Journal of comparative neurology. 2010;518(3):277-291.

5. Peterson BS, Thomas P, Kane MJ, et al. Basal Ganglia volumes in patients with Gilles de la Tourette syndrome. Archives of general psychiatry. 2003;60(4):415-424.

6. Albin RL, Young AB, Penney JB. The functional anatomy of basal ganglia disorders. Trends in neurosciences. 1989;12(10):366-375.

7. DeLong MR. Primate models of movement disorders of basal ganglia origin. Trends in neurosciences. 1990;13(7):281-285.

8. Alexander GE, DeLong MR, Strick PL. Parallel organization of functionally segregated circuits linking basal ganglia and cortex. Annual Review of Neuroscience. 1986;9:357-381.

9. Zhuang $\mathrm{P}$, Hallett $\mathrm{M}$, Zhang $X$, Li J, Zhang Y, Li Y. Neuronal activity in the globus pallidus internus in patients with tics. Journal of neurology, neurosurgery, and psychiatry. 2009;80(10):1075-1081.

10. Diagnostic and Statistical Manual of Mental Disorders: DSM-IV. Washington DC, American Psychiatric Association. 1994, ed 4.

11. Association AP. Diagnostic and statistical manual of mental disorders (DSM-5). 5th ed. Washington2013.

12. Robertson MM, Banerjee S, Kurlan R, et al. The Tourette syndrome diagnostic confidence index: development and clinical associations. Neurology. 1999;53(9):2108-2112.

13. Leckman JF, Riddle MA, Hardin MT, et al. The Yale Global Tic Severity Scale: initial testing of a clinicianrated scale of tic severity. Journal of the American Academy of Child and Adolescent Psychiatry. 1989;28(4):566-573.

14. Muller-Vahl KR, Cath DC, Cavanna AE, et al. European clinical guidelines for Tourette syndrome and other tic disorders. Part IV: deep brain stimulation. European child \& adolescent psychiatry. 2011;20(4):209-217.

15. Schrock LE, Mink JW, Woods DW, et al. Tourette syndrome deep brain stimulation: A review and updated recommendations. Movement disorders. 2014.

16. Ackermans L, Duits A, van der Linden C, et al. Double-blind clinical trial of thalamic stimulation in patients with Tourette syndrome. Brain. 2011;134(Pt 3):832-844.

17. Smeets AY, Duits AA, Plantinga BR, et al. Deep Brain Stimulation of the internal globus pallidus in refractory Tourette Syndrome. Clinical Neurology and Neurosurgery. 2016;142:54-59.

18. Nini A, Feingold A, Slovin H, Bergman H. Neurons in the globus pallidus do not show correlated activity in the normal monkey, but phase-locked oscillations appear in the MPTP model of parkinsonism. Journal of neurophysiology. 1995;74(4):1800-1805.

19. Raz A, Vaadia E, Bergman H. Firing patterns and correlations of spontaneous discharge of pallidal neurons in the normal and the tremulous 1-methyl-4-phenyl-1,2,3,6-tetrahydropyridine vervet model of parkinsonism. The Journal of neuroscience. 2000;20(22):8559-8571.

20. Hurtado JM, Gray CM, Tamas LB, Sigvardt KA. Dynamics of tremor-related oscillations in the human globus pallidus: a single case study. Proceedings of the National Academy of Sciences of the United States of America. 1999;96(4):1674-1679. 
21. Levy R, Hutchison WD, Lozano AM, Dostrovsky JO. Synchronized neuronal discharge in the basal ganglia of parkinsonian patients is limited to oscillatory activity. The Journal of neuroscience. 2002;22(7):28552861.

22. Alam M, Schwabe K, Lutjens G, et al. Comparative characterization of single cell activity in the globus pallidus internus of patients with dystonia or Tourette syndrome. Journal of Neural Transmission. 2015;122(5):687-699.

23. Hutchison WD, Lozano AM, Davis KD, Saint-Cyr JA, Lang AE, Dostrovsky JO. Differential neuronal activity in segments of globus pallidus in Parkinson's disease patients. Neuroreport. 1994;5(12):1533-1537.

24. Alam M, Sanghera MK, Schwabe K, et al. Globus pallidus internus neuronal activity: a comparative study of linear and non-linear features in patients with dystonia or Parkinson's disease. Journal of Neural Transmission. 2016;123(3):231-240.

25. Sanghera MK, Grossman RG, Kalhorn CG, Hamilton WJ, Ondo WG, Jankovic J. Basal ganglia neuronal discharge in primary and secondary dystonia in patients undergoing pallidotomy. Neurosurgery. 2003;52(6):1358-1370; discussion 1370-1353.

26. Starr PA, Rau GM, Davis V, et al. Spontaneous pallidal neuronal activity in human dystonia: comparison with Parkinson's disease and normal macaque. Journal of neurophysiology. 2005;93(6):3165-3176.

27. Vitek JL, Chockkan V, Zhang JY, et al. Neuronal activity in the basal ganglia in patients with generalized dystonia and hemiballismus. Annals of neurology. 1999;46(1):22-35.

28. Israelashvili M, Loewenstern Y, Bar-Gad I. Abnormal neuronal activity in Tourette syndrome and its modulation using deep brain stimulation. Journal of neurophysiology. 2015;114(1):6-20.

29. Yael D, Vinner E, Bar-Gad I. Pathophysiology of tic disorders. Movement disorders. 2015;30(9):11711178.

30. Baym CL, Corbett BA, Wright SB, Bunge SA. Neural correlates of tic severity and cognitive control in children with Tourette syndrome. Brain. 2008;131(Pt 1):165-179.

31. Peterson BS, Skudlarski $P$, Anderson AW, et al. A functional magnetic resonance imaging study of tic suppression in Tourette syndrome. Archives of general psychiatry. 1998;55(4):326-333.

32. Worbe $Y$, Malherbe $C$, Hartmann A, et al. Functional immaturity of cortico-basal ganglia networks in Gilles de la Tourette syndrome. Brain. 2012;135(Pt 6):1937-1946.

33. Albin RL, Mink JW. Recent advances in Tourette syndrome research. Trends in neurosciences. 2006;29(3):175-182.

34. Mink JW. Basal ganglia dysfunction in Tourette's syndrome: a new hypothesis. Pediatric neurology. 2001;25(3):190-198.

35. Israelashvili M, Bar-Gad I. Corticostriatal Divergent Function in Determining the Temporal and Spatial Properties of Motor Tics. The Journal of neuroscience. 2015;35(50):16340-16351.

36. McCairn KW, Bronfeld M, Belelovsky K, Bar-Gad I. The neurophysiological correlates of motor tics following focal striatal disinhibition. Brain. 2009;132(Pt 8):2125-2138. 



\section{Chapter 6}

\section{Modified cement-based fixation of the Deep Brain Stimulation electrode}

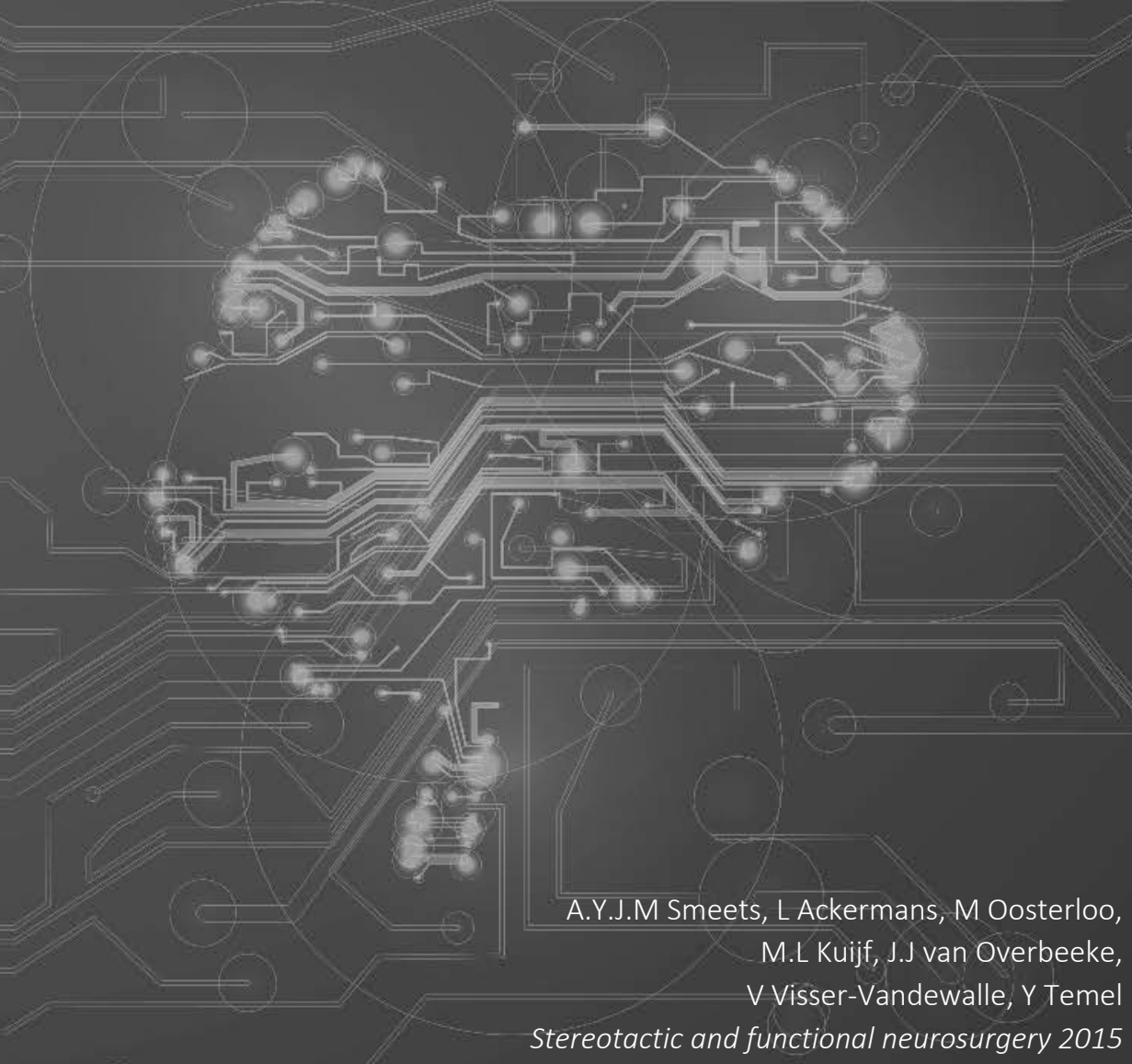



Deep Brain Stimulation (DBS) is becoming an increasingly performed surgery in daily neurosurgical practice. There are several important steps in DBS, such as patient selection, planning of the target, type of anaesthesia, microelectrode recording if performed, and the fixation of the final electrode (lead). Suboptimal fixation can result in electrode migration shortly after the surgery. Unintended migration, and consequently displacement of the electrodes, occurs in up to $10 \%$ of the cases and is a serious complication in DBS surgery ${ }^{1,2}$. This may result in no or suboptimal therapeutic effects, making a second surgery necessary ${ }^{1-3}$. There are generally two methods for the fixation of the electrode to the skull. The first is using a burr-hole device provided by the hardware manufacturers. For this, the form of the burr hole must be tailored to allow the placement of the burr-hole device ${ }^{3-5}$. The second is acrylic-based cementation ${ }^{4,6}$. This provides the surgeon with a certain degree of freedom in the choice of the form and size of the burr hole. Some of the acrylics contain antibiotics and may form a barrier against infections. In the past 15 years, we have been using antibiotic-containing acrylic $^{7}$. Besides some of its advantages, as outlined above, we experienced a disadvantage, which is the low degree of attachment of the acrylic to the bone, even with undercutting of the tabula interna to achieve a sandwich construction of the cement versus the burr hole. Frequently, we observed small movements of the acrylic construction related to the cerebrospinal fluid/parenchyma pulsations intraoperatively. To overcome this issue, we applied one to two anchoring titanium screws before applying the acrylic cement. This experience was derived from our laboratory studies, where we implanted cannulas and electrodes for long-term experiments and found out that anchoring screws are crucial in achieving a robust construction ${ }^{8}$. Here, we describe a rather simple modification of our previously reported antibiotic-containing cementbased fixation technique ${ }^{7}$ to achieve rigorous anchoring of the DBS electrode.

After having determined the optimal implantation site during DBS surgery, the microelectrode recording/test stimulation electrode is removed and fluoroscopy is performed. Then, the final DBS electrode is lowered to the optimal stimulation site. After placing two layers of oxidized cellulose polymer (Surgicel), one or two titanium screws ( $2 \times$ $5 \mathrm{~mm}$, the size might depend on individual preferences) are placed close to the burr hole and the acrylic is applied (Figure $1 \mathrm{~A}-\mathrm{C}$ ). Finally, the internal guide is removed and a second layer of acrylic is used to fix the electrode. We have performed eight surgeries (16 electrode fixations) with this method and have not observed any movements of the acrylic construct related to cerebrospinal fluid/parenchyma pulsations. The construction was extremely solid. This was independent of whether one or two screws were used. We did not compare the effectiveness of using one or two screws in a randomized fashion.

Adequate fixation of the DBS electrode is essential to prevent postoperative migration. The acrylic-based fixation method has been our method of choice for the last 15 years for several reasons. We modified this method by adding anchoring screws to increase the robustness. This change is in line with other attempts in the field to improve DBS surgery. 

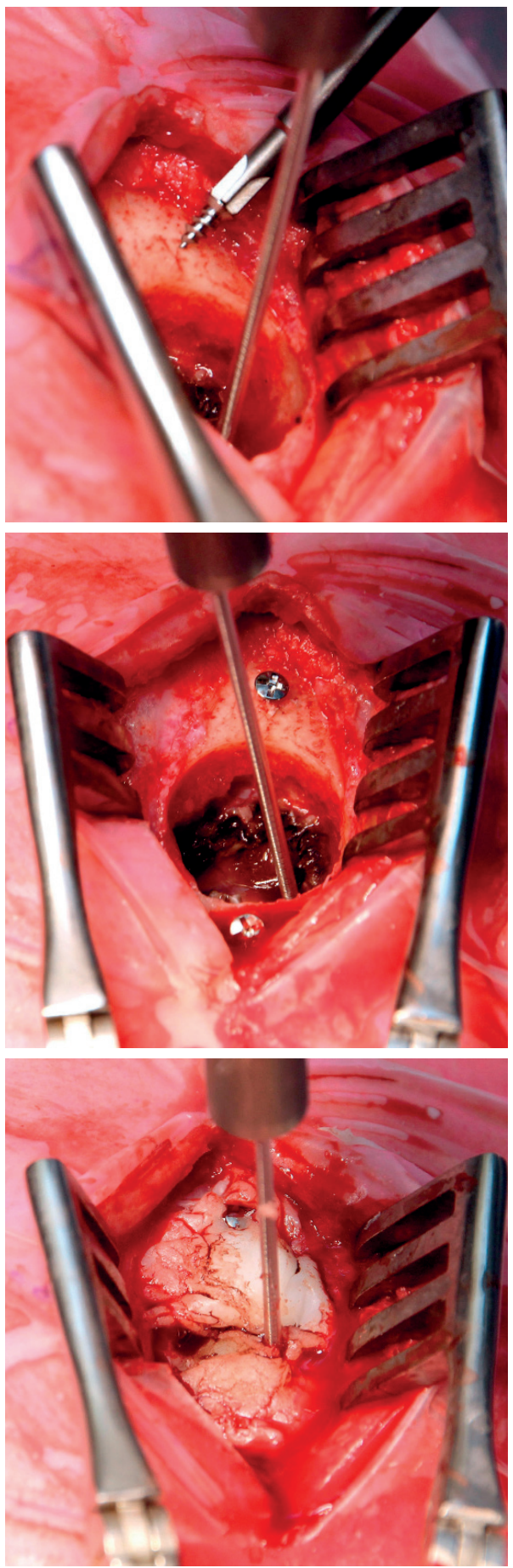

Figure 1A: Placement of one of the two screws partially in the skull, nearby the edge of the burr hole and in the opposite direction.

Figure 1B: Final position of the two screws which will connect the cement to the surrounding skull.

Figure 1C: The first layer of cement is placed and surrounds the electrode over the already inserted screws. 


\section{REFERENCES}

1. Baizabal Carvallo JF, Mostile G, Almaguer M, Davidson A, Simpson R, Jankovic J. Deep brain stimulation hardware complications in patients with movement disorders: risk factors and clinical correlations. Stereotactic and functional neurosurgery. 2012;90(5):300-306.

2. Doshi PK. Long-term surgical and hardware-related complications of deep brain stimulation. Stereotactic and functional neurosurgery. 2011;89(2):89-95.

3. Wharen RE, Jr., Putzke JD, Uitti RJ. Deep brain stimulation lead fixation: a comparative study of the Navigus and Medtronic burr hole fixation device. Clinical Neurology and Neurosurgergy. 2005;107(5):393-395

4. Lee MW, De Salles AA, Frighetto L, Torres R, Behnke E, Bronstein JM. Deep brain stimulation in intraoperative MRI environment - comparison of imaging techniques and electrode fixation methods. Minimimal Invasive Neurosurgery. 2005;48(1):1-6.

5. Ray CD. Burr-hole ring-cap and electrode anchoring device. Technical note. Journal of neurosurgery. 1981;55(6):1004-1006.

6. Favre J, Taha JM, Steel T, Burchiel KJ. Anchoring of deep brain stimulation electrodes using a microplate. Technical note. Journal of neurosurgery. 1996;85(6):1181-1183.

7. Kocabicak E, Temel Y. Deep brain stimulation of the subthalamic nucleus in Parkinson's disease: surgical technique, tips, tricks and complications. Clinical Neurolology and Neurosurgery. 2013;115(11):23182323.

8. Tan S, Vlamings R, Lim L, et al. Experimental deep brain stimulation in animal models. Neurosurgery. 2010;67(4):1073-1079. 



\section{Chapter 7}

\section{Ethics of Deep Brain Stimulation in}

adolescent patients with refractory Tourette

Syndrome: a systematic review and two case discussions

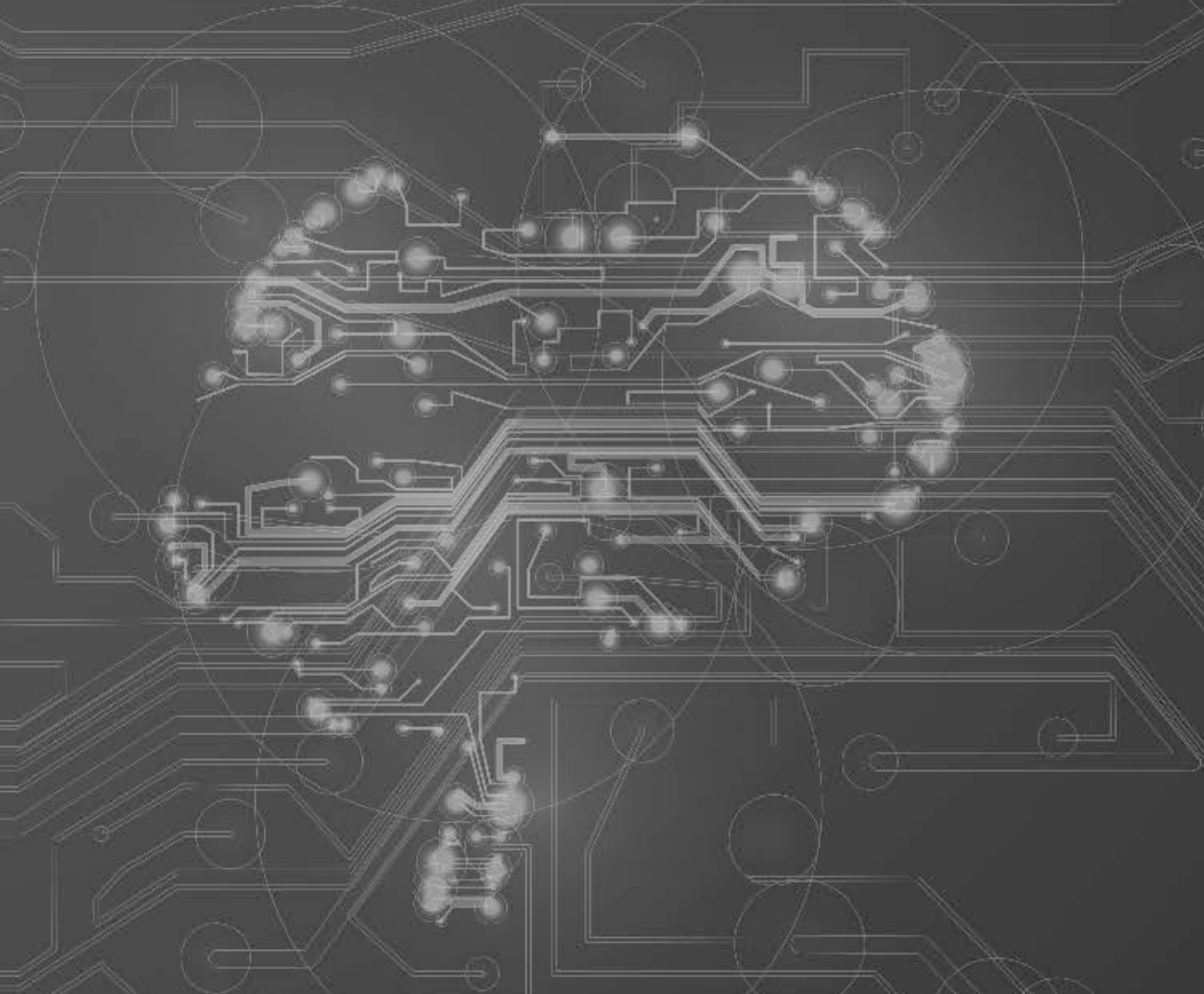

A.Y.J.M Smeets, A.A Duits, C Verdellen, G de Wert, D Horstkötter, Y Temel, L Ackermans, A.F.G Leentjens Neuroethics 2018 


\section{ABSTRACT}

Introduction: Tourette Syndrome is a childhood onset disorder characterized by vocal and motor tics and often remits spontaneously during adolescence. For treatment refractory patients, Deep Brain Stimulation may be considered.

Methods and results: We discuss ethical problems encountered in two adolescent Tourette Syndrome patients treated with Deep Brain Stimulation and systematically review the literature on the topic. Following surgery one patient experienced side effects without sufficient therapeutic effects and the stimulator was turned off. After a second series of behavioural treatment, he experienced a tic reduction of more than $50 \%$. The second patient went through a period of behavioural disturbances that interfered with optimal programming, but eventually experienced a $70 \%$ tic reduction. Sixteen Deep Brain Stimulation surgeries in adolescent Tourette Syndrome patients have been reported, none of which pays attention to ethical aspects.

Discussion: Specific ethical issues arise in adolescent Tourette Syndrome patients undergoing Deep Brain Stimulation relating both to clinical practice as well as to research. Attention should be paid to selecting patients fairly, thorough examination and weighing of risks and benefits, protecting the health of children and adolescents receiving Deep Brain Stimulation, special issues concerning patient's autonomy, and the normative impact of quality of life. In research, registration of all Tourette Syndrome cases in a central database covering a range of standardized information will facilitate further development of Deep Brain Stimulation for this indication.

Conclusion: Clinical practice should be accompanied by ongoing ethical reflection, preferably covering not only theoretical thought but providing also insights in the views and perspectives of those concerned, that is patients, family members and professionals. 


\section{INTRODUCTION}

Tourette Syndrome (TS) is a childhood onset neuropsychiatric disorder with multiple motor tics and one or more vocal tics, lasting longer than one year. It affects approximately $0.3-1.0 \%$ of the general population ${ }^{1-3}$. The majority of patients suffer from associated comorbid conditions such as Attention Deficit and Hyperactivity Disorder (ADHD), Obsessive-Compulsive behaviour (OCb), anxiety, and affective disorders ${ }^{4}$. Typically, tics start around the age of 4-6 years, multiply and worsen until the age of 12-14 years and are then followed by a steady decline in severity. TS is often self-limiting, with symptoms remitting around the late adolescence. However, in about $20 \%$ of patients, tics continue in adult life and require chronic treatment ${ }^{1,3}$. Despite behavioural therapy or pharmacological treatment, a small subgroup of patients fails to respond to treatment and continue to experience significant symptom burden throughout life ${ }^{5-7}$. For those patients, Deep Brain Stimulation (DBS) surgery can be an effective last resort treatment ${ }^{8}$. It must be realized however, that TS is not (yet) a recognized indication for DBS treatment by the Food and Drug Administration (FDA) and the European Commission (EC). We have a longstanding experience with DBS surgery in TS patients since in our centre we performed 16 surgeries in the past 18 years, which is $10 \%$ of all reported cases worldwide ${ }^{9-13}$.

The emergence of psychiatric disorders, such as depressive disorder, Alzheimer's disease and anorexia nervosa, as potential indications for DBS as well as the application of DBS in younger patients has created new interest in ethical questions and social challenges ${ }^{14,15}$. For most of these indications, ethical considerations have been published ${ }^{16-18}$. To date, no ethical considerations have been published on DBS in TS patients, let alone for younger TS patients. The minimum age for considering DBS in TS patients has been widely debated. In 2006, the first guidelines of the Tourette Syndrome Association (TSA) ${ }^{19}$ proposed a minimum age of 25 years to ensure that individuals who might experience spontaneous tic remission would not be implanted with a surgical device. More recently, compelling arguments have been made for consideration of surgical intervention at younger ages in certain cases of severe TS. Especially during adolescence, the impact of severe and refractory tics on daily functioning is large. Patients are often not able to go to school or work, they tend to avoid social activities, are not able to develop friendships or an affective relationship, and have a low self-esteem. This developmental interference may have long-lasting effects in later life. Another reason to consider DBS earlier in life is the risk of selfinjurious tics, e.g. violent cervical tics which may cause cervical myelopathy and secondary neurological deficits ${ }^{20-22}$. This form is sometimes called 'malignant' TS and occurs in about $5 \%$ of patients ${ }^{23}$. Delaying or not performing DBS in these patients could eventually lead to irreparable harm. Therefore, in more recent TSA recommendations ${ }^{24}$ the age guideline has been adjusted and they recommend a multidisciplinary evaluation and discussion, without setting a strict minimum age. 
This paper deals with ethical issues associated with DBS of adolescent TS patients. Adolescence is the period of transition from childhood to adulthood. The World Health Organization (WHO) defines adolescence as the age period between 10 and 19 years ${ }^{25,26}$, however the period of adolescence has often been extended to include the ages of 10 through 22 - 25 years, with most researchers dividing this age span into early $(10-13)$, middle $(14-17)$ and late $\left(18\right.$ - mid-20s) adolescence ${ }^{27}$. Therefore, we define adolescence as the age period between 10 and 25 years in this paper. We describe our experiences with the treatment of two adolescent TS patients, both 19 years old, and systematically review the scarce literature on this subject. We discuss the ethical issues and specific difficulties that physicians may encounter when treating adolescent patients.

\section{METHODS AND RESULTS}

\section{Patient 1}

Patient 1 is a 19-year-old male who experienced tics since the age of five. No other diseases or comorbidities were present, and he had a negative family history for tic syndromes. The tics started with sniffing sounds and tapping his chest, but gradually expanded and increased over the years. At the age of 18, vocal tics, such as almost continuous and loud barking and coprolalia, were the most debilitating. He also suffered from frequent motor tics such as eye blinking and head shaking. Because of the disruptive nature of the tics, he had to leave school and avoided social activities. Different pharmacological treatments as well as behavioural therapy had not been effective in the past. From 2005 till 2013 he used clonidine, risperidone, pimozide and aripiprazole, either solely or in combination, without a positive effect on tics and/or with severe side effects. Moreover, botulin injections did not result in tic improvement. In 2010, he received cognitive behavioural therapy for several months without any improvement. He fulfilled all our criteria for DBS surgery (Table 1), which are in line with the TSA guidelines for DBS in TS ${ }^{24}$. After a multidisciplinary evaluation, he was indicated for bilateral DBS of the anterior internal globus pallidus (GPi) and the surgery was carried out successfully in March 2014 (at the age of 19). Details about the neurosurgical procedure have been published previously ${ }^{10,11}$. Preoperative he scored $39($ motor/vocal $=14 / 25)$ on the Yale Global Tic Severity Scale $(\text { YGTSS })^{28}$.

A few days after surgery, the stimulator was turned on and different contact points and stimulation parameters were tested. With increasing voltages, he experienced side effects such as hyperkinesia, dyskinesia in the legs and a dejected mood, complicating the programming. Therefore, the voltage was reduced before he was discharged, accepting a suboptimal tic reduction at that moment. The expectation was that these side effects would diminish due to habituation and that the voltage could be increased 
slowly at the outpatient department. However, after six months of intensive outpatient visits and a second admission at the hospital to systematically check all possible stimulation parameters, he still experienced the same side effects at all contacts without sufficient therapeutic effects. We decided to turn the stimulator off. At that time, his score on the YGTSS was 24 (motor/vocal =10/14), which was a relevant reduction compared to the pre-operative score. However, he still suffered from severe tics, e.g. constantly loud barking and headshaking, he felt ashamed and avoided social activities. In September 2016, at the age of 21, he started behaviour therapy again for three months with a further relevant improvement in tics, self-confidence and overall functioning. His YGTSS further declined to 18 (motor/vocal = 8/10), two years after surgery. In a last outpatient appointment, he was satisfied with his current disease severity and stated that with this symptom severity he would in retrospect not have considered DBS. At this moment, he does not want the stimulator removed since he experiences no burdens and does not want to undergo surgery again.

Table 1: Inclusion and exclusion criteria for Deep Brain Stimulation in Tourette Syndrome.

\begin{tabular}{|c|c|}
\hline Inclusion criteria * & Exclusion criteria * \\
\hline $\begin{array}{l}\text { - Primary diagnosis of TS according to the DSM-V } \\
\text { criteria } 57 \\
\text { - A minimum score of } 80 \text { on the Diagnostic Confidence } \\
\text { Index }{ }^{58} \\
\text { - A minimum score of } 25 \text { on the Yale Global Tic } \\
\text { Severity Scale } 27 \\
\text { - Failure to respond to, or intolerable side effects of } \\
\text { three-months trials of adequately dosed classical } \\
\text { (e.g. haloperidol) and atypical (e.g. risperidone, } \\
\text { olanzapine, quetiapine) antipsychotic medication } \\
\text { or clonidine } \\
\text { - Completed at least ten sessions of behavioural } \\
\text { therapy (e.g. exposure and response prevention } \\
\text { or habit reversal) } \\
\text { - A stable psychosocial environment } \\
\text { - Neuropsychological profile indicates candidate can } \\
\text { tolerate demands of surgery, postoperative } \\
\text { follow-up, and possibility of poor outcome } \\
\text { - Age is not a strict criterion. Local ethics committee } \\
\text { involvement for cases involving persons < } 18 \text { years, } \\
\text { and for cases considered "urgent" (e.g. impending } \\
\text { paralysis from head-snapping tics) }\end{array}$ & $\begin{array}{l}\text { - Tics not related to TS } \\
\text { - Major psychiatric disorders (e.g. schizophrenia or } \\
\text { bipolar disorder). } \\
\text { - Current substance abuse of dependence (except } \\
\text { for nicotine) } \\
\text { - Severe cognitive impairment } \\
\text { - Structural abnormalities on brain Magnetic } \\
\text { Resonance Imaging } \\
\text { - General contraindications for surgery or } \\
\text { anaesthesia }\end{array}$ \\
\hline
\end{tabular}

* These criteria are in line with the latest worldwide recommendations for DBS in Tourette Syndrome ${ }^{24}$. DBS: Deep Brain Stimulation; TS: Tourette Syndrome; DSM-V: Diagnostic and Statistical Manual of Mental Disorders criteria (fifth edition).

\section{Patient 2}

Patient 2 is a 19-year-old male adolescent that developed his first motor tics at the age of eight. No other comorbidities were present, and he had a negative family history for 
tic disorders. Tics started with head shaking, followed by eye blinking and grimaces, and later progressed to his shoulders, arms, trunk and legs. The most debilitating tics were persistent bouts of motor tics in his entire body. He also experienced mild vocal tics like coughing, sniffing and grumbling. At the age of 18 , he had to quit school since he was not able to concentrate anymore due to the severity of the tics and because his tics were an unacceptable distraction to fellow students. Medication and behavioural therapy had not been effective in the past. He had used clonidine, pimozide, haloperidol and quetiapine either without lasting positive effects or with intolerable side effects. Between 2007 and 2009 he underwent several psychotherapeutic therapies, among which exposure and response prevention, habit reversal and relaxation training. He was using cannabis daily since he felt it calmed him. This patient fulfilled our selection criteria (Table 1) and was indicated for bilateral DBS of the anterior GPi. He agreed to stop using cannabis as part of the treatment agreement, so that better evaluation of symptoms during programming would become possible. The surgery was carried out without complications in March 2016 (at the age of 19).

Within one week after the surgery the stimulator was turned on and systematically different stimulation parameters were tested. Initially he experienced increased agitation, but also an increase in tic frequency and severity. Despite he was informed that finding the correct stimulation parameters would take time, he had hoped for an immediate effect on his tics and was disappointed. This resulted in tension, anger and arguments with his supporting parents, making the programming sessions more difficult. In the following weeks, we had several programming sessions at the outpatient department, mainly decreasing the voltage and slowly increasing it at home, and finally with a voltage of 3.0 a significant tic reduction was established and the agitated feeling had almost disappeared. However, at home the situation escalated, mainly due to the daily excessive use of cannabis, with increasingly quarrelsome and aggressive behaviour. He still lived at home with his parents and siblings who tried to support him and this lead to severe conflicts. The exact reason why he started using drugs again is uncertain, but he told us he was overwhelmed, felt a lot of pressure to succeed with the therapy, and needed the cannabis to calm down, even though he knew it caused him more problems. We offered psychological treatment, which he rejected.

In July 2016, we arranged a meeting with the Committee on Medical Ethical Issues of our hospital to discuss how to proceed: we were not able to reliably evaluate the effects of the DBS due to the excessive use of cannabis and non-compliance of the patient and doubted whether continued treatment was useful. Also, the potential contribution of the DBS to the aggressive behaviour was discussed. He was known with aggressive behaviour following cannabis use and it seemed unlikely that the DBS settings were the cause of his behavioural problems. Therefore, we decided to continue treatment because of its positive effect on tics. However, we discussed with the patient that we would not further adjust the stimulation settings if he would not be compliant with all aspects of the treatment agreement, including stopping cannabis usage. A few 
months later, his behaviour drastically changed in a positive way. He stopped smoking cannabis, his aggressive behaviour as well as the relationship with his parents improved, and he found a job and started working again. One year after surgery, he was quite satisfied with the effects of DBS. All tics were still present, but much less frequent and intense and he could suppress them better. He did not suffer from side-effects anymore. At that moment, his YGTSS was reduced from 32 preoperatively (motor/vocal $=23 / 9$ ) to 10 postoperatively (motor/vocal $=8 / 2$ ).

\section{Overview of the ethical literature}

We systematically searched the literature for papers discussing ethical issues in young TS patients treated with DBS. We searched PubMed with the search strategy; (Tourette's syndrome OR Tourette syndrome OR Gilles de la Tourette syndrome OR Tourette's disorder OR Tourette OR tic disorder) AND (Deep brain stimulation OR DBS) without any time or language restriction (Figure 1).

Since 1999, 156 cases of DBS treatment in TS patients have been published ${ }^{29}$. Of these, only 16 surgeries in patients under 25 years of age have been described in case reports (Table 2) ${ }^{20-22,30-40}$. These reports mainly focus on target selection, stimulation parameters, and effects on tics and comorbid psychiatric disorders. The most common reasons for operating on these young patients were the severity and intractability of the disease, the considerable influence on daily functioning (e.g. not able to go to school or work, avoiding social activities, not able to develop friendships, and low self-esteem), and severe motor tics causing self-injurious behaviour 20-22,32-35,37,39,40. None of these reports discussed the ethical aspects of DBS in adolescent patients. Moreover, minimal attention was paid on to the specific difficulties encountered in these younger patients.

Most research groups used the minimum age of 25 years as an inclusion criterion for surgery, but some groups did not follow this advice and therefore larger studies might have also included some younger patients ${ }^{41-50}$. In the latest TSA guidelines, 33 patients were reported that had DBS before the age of 25 years. The risk of surgical complications and adverse events did not appear to be higher in this age group compared with older patients ${ }^{24}$. Servello et al ${ }^{42}$ concluded that in their series of 18 patients implanted with thalamic DBS electrodes, three of the four cases who were implanted before the age of 20 years had less satisfactory results after 3 to 6 months than those older than 20 years. This was most likely due to spontaneous waxing and waning of symptoms requiring frequent DBS programming. So far, detailed case descriptions are lacking and no attention has been paid on the ethical issues and specific difficulties that physicians may encounter when treating adolescent TS patients. As such we conclude that there is yet no literature on ethical aspects of performing DBS on TS patients, let alone on adolescent TS patients. 


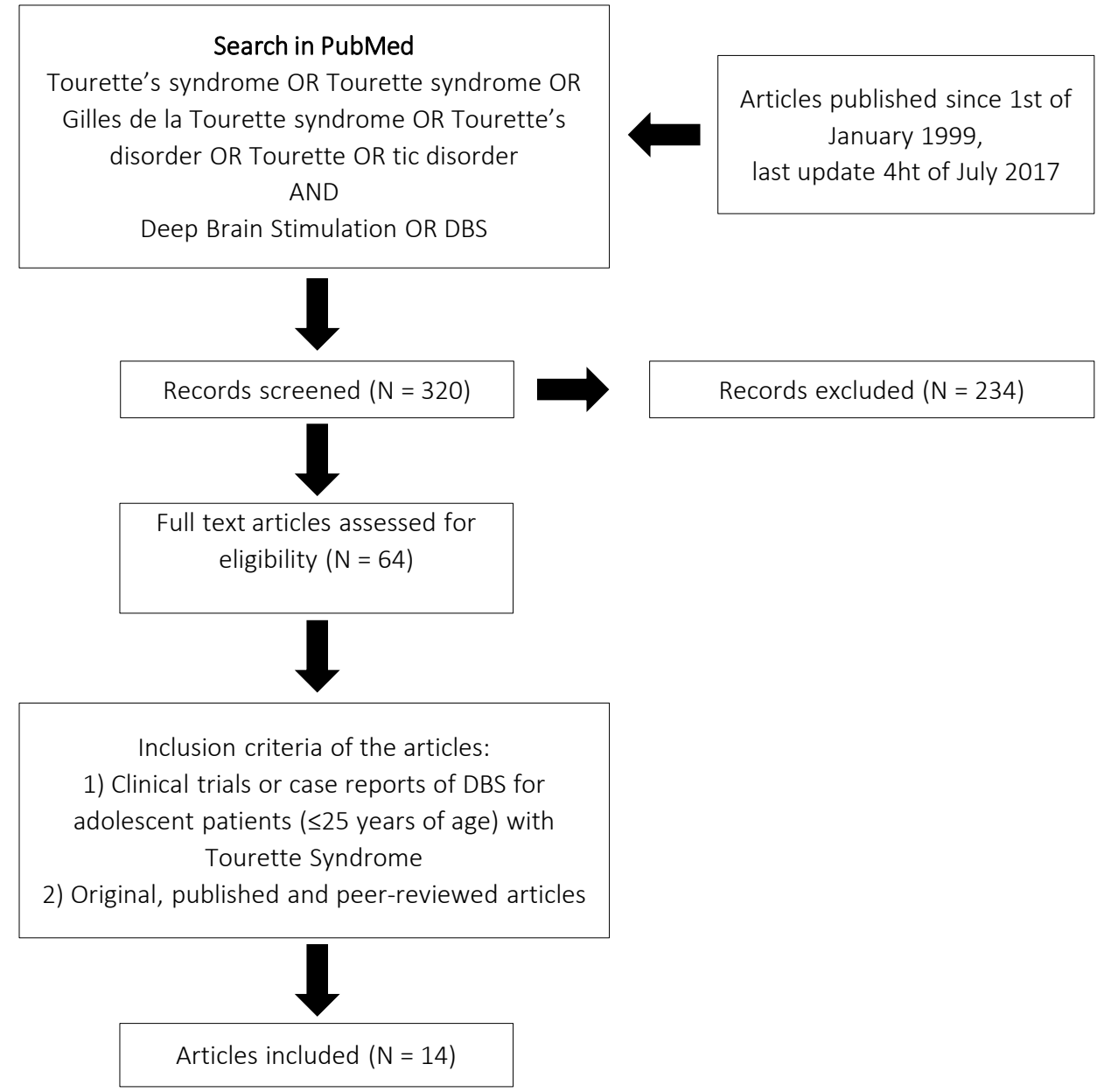

Figure 1: Literature search. 


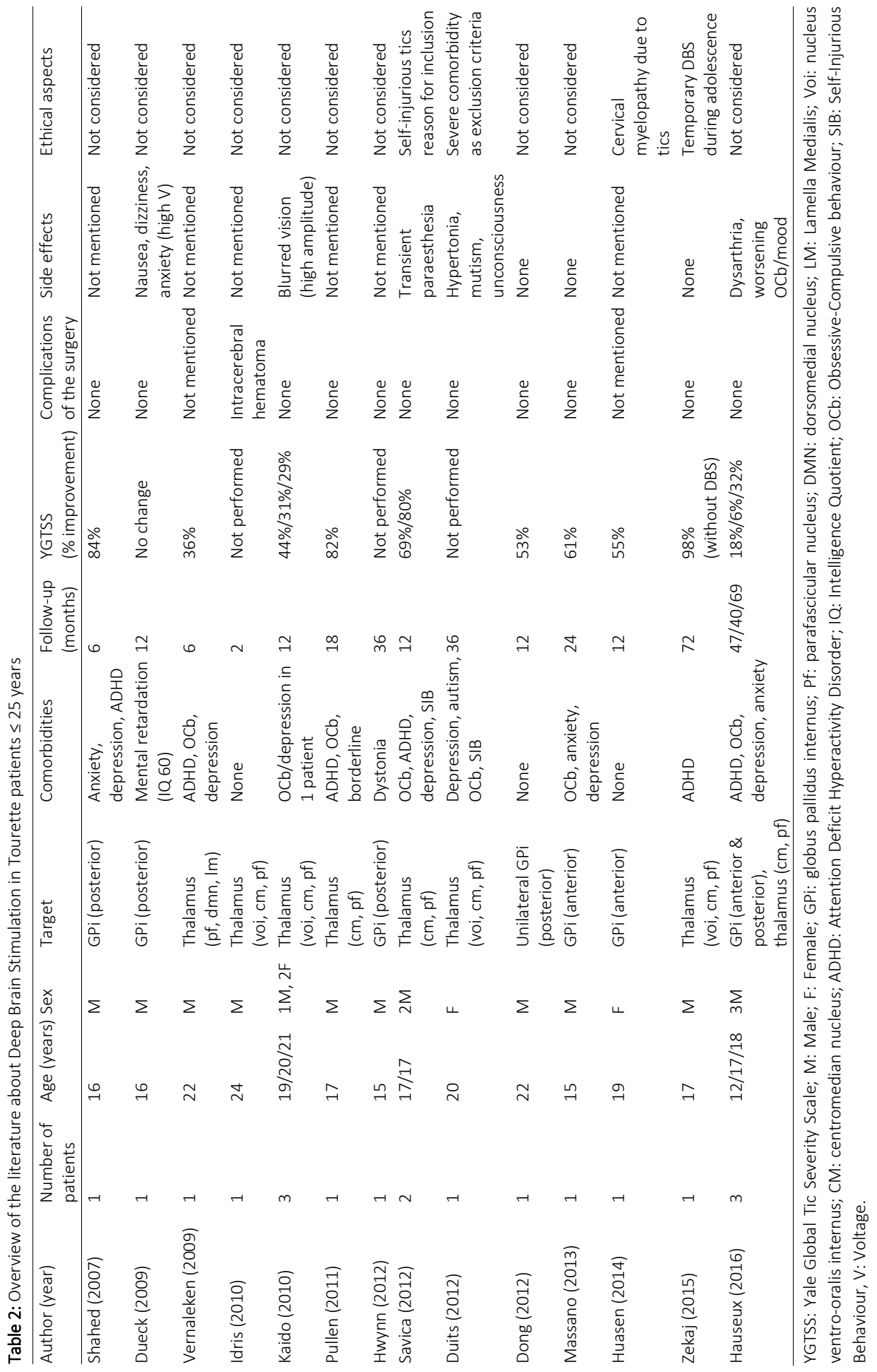




\section{DISCUSSION}

We have described our experiences with DBS in two severely affected and refractory adolescent TS patients. Three years after surgery one patient experienced a tic reduction of more than $50 \%$ with the stimulator turned off. The reduction may be due to natural waning of tics that often follows adolescence and due to an additional behavioural treatment for tics after the DBS. At present, he is satisfied with the level of symptoms and would not consider the surgery anymore. The second patient is responding well to the stimulation, but went through a period of behavioural disturbances which are not uncommon during adolescence but interfered with optimal programming. We believe that the experienced stress before, around, and after the surgery might have provoked the excessive use of cannabis and caused the related behavioural disturbances.

DBS in TS seems to have a promising future in terms of tic reduction, however, it raises several ethical questions that need continuing discussion especially in adolescent patients. Five major topics concerning the ethics of DBS in clinical practice have been identified previously: 1) selecting patients fairly, 2) thorough examination and weighing of risks and benefits, 3) protecting the health of children in paediatric DBS, 4) special issues concerning patient's autonomy, and 5) the normative impact of quality of life measurements ${ }^{51}$.

When discussing the first topic. selecting patients fairly, one of the first questions is whether DBS should be indicated for adolescent patients with TS, given the chances of natural remission during adolescence. In $40 \%$ of TS patient's tics decrease during adolescence, in another $40 \%$ they may disappear completely and only in $20 \%$ they remain in their full intensity and require chronic treatment ${ }^{1,3}$. Chronic treatment may include pharmacotherapy and behavioural or psychosocial therapy ${ }^{6,7}$, but there is a small subset of patients who fail to show clinical improvement, and experience intolerable side-effects or potentially life-threatening tics or self-injurious behaviour. These patients are potential candidates for a surgical intervention. The available research shows that DBS of different targets in TS is effective and results in a mean tic improvement of $53 \%$ on the YGTSS ${ }^{29}$.

Based on the new insights and guidelines we have waived our previous age limit of 25 years ${ }^{24}$. This brings us to incorporating the second topic. thorough examination and weighing of risks and benefits. Our motivation for waiving this age limit was that severe TS in adolescence can be severely disruptive to development, often jeopardizing educational and job opportunities, social interactions and relationships. Delaying surgery in these younger incapacitated TS patients could potentially result in permanent harm to social, psychological, and intellectual development, even if the symptoms eventually subside with age. Similarly, in rare cases of 'malignant TS', the tics themselves may carry greater risk for bodily harm or even death 20-22,24. An early intervention with positive results may bring significant benefits on the individual's 
development and future. On the other hand, DBS is an invasive treatment with potential surgical complications and adverse events. Stimulating the anterior GPi has been related to side-effects such as higher anxiety levels, dyskinesia, fatigue, dizziness and a case of hypomania ${ }^{29,52}$. Stimulating different targets can cause other side-effects such as gaze disturbances, mood deterioration, apathy and dysarthria ${ }^{29}$. In many instances adjustment of the stimulation parameters can diminish or eradicate these side-effects. Surgical complications mostly centre around hardware malfunction and infections ${ }^{53,54}$. In general DBS is considered as a safe surgical procedure with a minimal risk $(<1 \%)$ of serious complications (e.g. bleeding, neurological deficits) ${ }^{24,29,53}$. Thorough selection of patients who should be offered DBS is an essential requirement for a successful intervention from a clinical as well as an ethical perspective. In general, patient selection should optimize the individual risk-benefit ratio ${ }^{51}$.

Both our patients suffered from severe and refractory TS for many years and were selected for DBS after a thorough multidisciplinary evaluation. Patient 1 had received behavioural treatment and several medications without success and hence he met the DBS inclusion criteria. After unsuccessful DBS, he tried a behavioural treatment again, from which he reported a positive effect on tic severity, self-confidence and quality of life. Behaviour therapy implies doing homework exercises, which this patient reported he had not done the first time he underwent this treatment, because at that time he could not focus on this treatment since he was too busy with school and his parents were in a divorce. Although this patient met the inclusion criteria for DBS, an additional step before starting DBS could have been thorough evaluation of which interventions for tics have been done before and why the patient did not profit from it. After optimizing these first-line less invasive interventions [7] DBS might be an option for further treatment.

A gap in our current state of knowledge is that it is not possible to predict the natural course of tics in TS, so that expected chronicity cannot be used to indicate DBS treatment. At present it remains unclear whether (and to what extent) severely affected patients at the age of 18 may or may not await a significant improvement until the age of $25^{8}$. Clinicians selecting DBS candidates would benefit from knowledge of predictive factors, both epidemiological as well as biomarkers, that would allow identification of patients who will remain severely affected into adulthood, but so far this is not possible. Therefore, the advantages and disadvantages of DBS should be evaluated multidisciplinary for every individual adolescent TS patient who may be a surgical candidate. Our patients were informed about the possibility of natural improvement or remission and the possibility of limited or no effect of the surgery on tics. Apparently, they chose to have the operation because of their actual suffering, and not because of the chance of chronicity. In patient 1 as well as in the case described by Zekaj et al ${ }^{40}$, tic severity significantly declined during adolescence, to such an extent that the stimulator in these patients was turned off and they would not be eligible for DBS anymore. Should we not have operated on these patients? With the knowledge of hindsight probably not, but that does not help in the decision making beforehand. On the other hand, if 
DBS resulted in positive stimulation effects in this very important period of life, would it be ethical to deprive them from this therapeutic option? Moreover, would it be ethical to consider DBS a temporary therapeutic application? And if so, should we remove the stimulator when, after turning off the system, there is no increase in tics for a certain period? It is important to realize that since DBS may be an effective procedure in selected treatment-resistant adolescent TS patients, one may do harm to patients not only by performing DBS, but also by not performing it. It seems to be justified to leave traditional reticence (based on the substantial chance of natural remission) behind and allow earlier intervention in view of the combination of acute suffering and the chance of irreparable harm. The patient's benefit is central in the ethical evaluation. This criterion can outweigh risks and side effects, and can make DBS appropriate even in adolescent TS patients with a chance of natural remission ${ }^{51}$.

The third topic is protecting the health of children in paediatric DBS. This can be expanded to protecting the health of adolescent TS patients selected for DBS (age 10 25 years). Local ethics committee involvement is necessary for cases involving persons under 18 years and for cases considered "urgent" in our centre ${ }^{24}$. Moreover, following all the in- and exclusion criteria and basing the decision on a multidisciplinary decision is mandatory. Successful application of the DBS procedure requires much more than the surgery itself. In addition, guidance before, during and after surgery and defining realistic expectations are of major importance. Programming the stimulator to gain the settings with the best effects on tics and no or minor side-effects may take months to a year ${ }^{24}$. This is clearly different from, for example, Parkinson's disease or essential tremor in which an immediate effect can be observed. Significant spontaneous waxing and waning of symptoms in TS requiring frequent DBS programming makes it even more difficult. The influence of this might be greater during adolescence and spontaneous waning may even be a first sign of spontaneous remission ${ }^{1,55}$. Therefore, the inclusion criteria for surgery should go much further than being purely clinical criteria and consider certain psychological, social and environmental factors too. To get through all the problems and challenges (e.g. school issues and interpersonal relationships) during the period from childhood to adulthood and handle the related stress, it is very helpful for adolescents not only to have the understanding and support from peers and adults, but also to be able to manage the stressors and hassles of everyday life by themselves. The development of coping strategies may support or inhibit positive adolescent adaptation.

Adolescents who lack adequate coping strategies are more likely to show deviant behaviour (e.g. substance use) and use avoiding and distancing coping strategies, whereas adolescents with healthy coping try to solve the problems or seek for social support ${ }^{56}$. Ideally, adolescent candidates for DBS surgery have a stable psychosocial environment and an effective and positive coping style. Patients should be able to tolerate the demands of surgery and the postoperative follow-up, and also the ability to cope with the possibility of a poor outcome. As such it is important for all DBS centres who operate on 
TS patients with a multidisciplinary approach to include an extensive (neuro)psychological evaluation. Since adolescent patients often still live with their parents, a supporting attitude of parents and other relatives is also of significant importance. Even though formally informed consent lies with the young patient only, especially these young patients can profit from an approach in which both the patient and parents, or other close caregivers, are consulted during the DBS trajectory and involved in treatment and followup. In patient 2 the stress experienced before, around and after the surgery might have provoked the excessive use of cannabis and the related behavioural disturbances as a result of an avoiding coping style. Psychological support already prior to surgery can help those patients and parents with a less effective coping style to deal with the stress that can accompany the surgery. Psychological support is also helpful to improve overall compliance and other difficulties that may impede successful DBS.

The fourth topic concerns the patient's autonomy and the decision for surgery should be based upon autonomous decision-making of the potential patient ${ }^{57,58}$. Respect for patient's autonomy is expressed by safeguarding for informed consent. Some aspects of DBS should be given special attention, namely the complexity of the treatment, the expectations of the patient and his relatives, compliance, and the operating technique ${ }^{51}$. A specific problem in the latter context is the so-called therapeutic misconception, which implies that patients expect evident personal benefit, but fail to realize the innovative character of the treatment and undervaluing the fact that individual treatment response is uncertain and long-term cognitive, emotional and behavioural effects are largely unknown. Desperation is often very high in severely affected TS patients which increases to hope for improvement by DBS. Moreover, depression occurs frequently in adolescent TS patients ${ }^{59}$, and their preferences can be more strongly influenced by affective components. Combined, this could lead to the danger of overhasty decisions in favour of undergoing DBS surgery. In addition to informed consent, DBS could have an impact on patient's autonomy in a separate way. Faced with decision conflicts DBS patients might fail to slow down and make impulsive decisions for their disadvantage ${ }^{51}$. This might have played a role in our second patient. Although he was informed that finding the correct stimulation settings would take time and that we did not expect an immediate effect on his tics, he was very disappointed. This disappointment became part or maybe even the cause of the behavioural problems complicating the treatment even more. Moreover, a stable psychosocial environment, such as supportive parents, is one of the inclusion criteria for DBS and especially important in adolescent patients. Despite being of importance for a positive outcome of DBS, this same environment could also undermine autonomous decision making of the patient. Adolescence is a period of understanding the 'self' in relation to the social world with a desire to find a balance ${ }^{26}$. Adolescents can base their decision to take part and undergo treatment on external forms of pressure, either perceived or actual. They may feel ashamed and a burden to their family. These feelings may make them suffer even more than the tics themselves. This may influence their decision to participate and 
undermines autonomous decision-making and voluntary participation. To address these issues, a good relationship between researcher and patient is essential, given also that life-long treatment and follow-up within the same institution will be needed.

DBS is an elective intervention in TS that is neither life-saving, nor can it cure the disease, and the goal for patients is usually to improve their quality of life, which is greatly limited in refractory TS patients. This brings us to the fifth topic the normative impact of quality of life measurements. Enquiring for the quality of life of the patients in treatment, additionally to the effectiveness of the intervention, is an important aspect from an ethical point of view. In Parkinson's disease and other movement disorders, the pure effectiveness of the intervention regarding the motor functions has been considered normatively meaningless ${ }^{51}$. At this point, the question rises whether this also holds in case of TS. In patients with TS the deterioration of their quality of life does not merely consist in the very fact that they do experience these tics, but also in the negative effects the tics have on their surroundings as well as reactions of other people on the tics, resulting in low self-esteem. In addition, DBS treatment in adolescent TS patients has a normative impact on young patient's long-term quality of life comparable to those described for patients with movement disorders. Delaying the surgery might to lead to deterioration of quality of life during this important period of life. Even though the effects of DBS might be even successful several years after adolescence, irreparable personal and social harm might be done. Tics that are experienced as insulting or disturbing, for example coprolalia or severe motor tics in class room settings, are normatively meaningful because they also affect other persons. Such social consequences and the reactions of others contribute to the fact that young TS patients end up with having their educational and job opportunities ruined and their social interactions and relationships potentially even irreparably harmed. Therefore, the effectiveness of the DBS intervention might be normatively meaningful in itself. The first patient experienced an improvement in tics and quality of life three years after surgery, which seemed to be independent of the DBS. The second patient experienced a beneficial effect on his tics which allowed him to start working again and to improve his social contacts, eventually leading to an improvement of his quality of life. At the same time, as discussed before, normatively relevant risks and benefits go further than effectiveness. Too high expectations, disappointment in case there is no immediate success, great suffering pressing patients to seek solutions, troubles with coping with surgery- and treatment-related stress, are all issues that are relevant in this regard.

Although ethical criteria for evaluating psychiatric DBS are principally similar to those discussed for other movement disorders, the experimental character of DBS in TS raises additional questions of research ethics. DBS for TS is not (yet) a recognized indication by regulating authorities and it must be considered a treatment that should only be done in a research setting. This triggers several structural challenges that DBS trials in this domain will unavoidably face. In addition to safeguarding the rights and interests of vulnerable research participants, it would be important to initiate set-up and promotion of high- 
quality scientific research that could serve the interest of future patients ${ }^{57}$. Today, such trials are not performed worldwide, and such a trial set-up faces several challenges. Even experienced DBS centres perform only a handful of DBS surgeries in TS patients each year, using different techniques and targets, making it difficult to obtain and interpret large-scale outcomes. Multicentre randomised controlled trials would theoretically be desirable, but difficult to realize due to practical reasons and would stretch over many years, and often do not provide conclusive evidence in heterogeneous populations, such as TS patients ${ }^{50}$. Currently the inclusion process, surgical techniques, targets, programming and postoperative care differ largely between different institutions and countries. Moreover, during such study new insights might be gained, e.g. pointing to different surgical targets or other changes in the procedure, which would be unethical to withhold from later participants. Because of these limitations, coupled with the variable effect of surgery, and the overall small numbers of TS patients with DBS worldwide, there is no regulatory agency approval from the FDA and the EC to date. To overcome these structural limitations and to take steps towards larger scale analyses, the TSA recently launched an international Tourette DBS registry and database ${ }^{60}$. The main goal of the project is to share data, uncover best practices, improve outcomes, and provide critical information to regulatory agencies. Such a database can also allow for an analysis of adolescent TS patients to determine for which group of younger patients DBS would be beneficial, either temporarily or lifelong lasting.

\section{CONCLUSION}

DBS surgery for TS patients has lately been considered at an earlier age because more stress has been placed on the potential long lasting harmful effects of the disorder. Specific ethical issues arise in this age-group relating both to clinical practice as well as to research. In clinical practice attention should be paid to selecting patients fairly, thorough examination and weighing of risks and benefits, including evaluation of adequacy of former applied first-line interventions, protecting the health of children and adolescents receiving DBS, special issues concerning patient's autonomy, and the normative impact of quality of life. Realistic expectations, consideration of the psychological, social and environmental conditions of adolescent TS patients and the adequate inclusion of relatives in the process of decision-making and care are important. In research, registration of all TS cases in a central database covering a range of standardized information will facilitate the further development of DBS for this indication. Clinical practice should be accompanied by ongoing ethical reflection, preferably covering not only theoretical thought but providing also insights in the views and perspectives of those concerned, that is patients, family members and professionals. Examining the ethical issues of DBS may help to realize its entire potential for benefiting severely suffering TS patients. 


\section{REFERENCES}

1. Bloch MH, Leckman JF. Clinical course of Tourette syndrome. Journal of psychosomatic research. 2009;67(6):497-501.

2. Robertson MM. The prevalence and epidemiology of Gilles de la Tourette syndrome. Part 1: the epidemiological and prevalence studies. Journal of psychosomatic research. 2008;65(5):461-472.

3. Robertson MM. A personal 35 year perspective on Gilles de la Tourette syndrome: assessment, investigations, and management. Lancet Psychiatry. 2015;2(1):88-104.

4. Robertson MM. Tourette syndrome, associated conditions and the complexities of treatment. Brain. 2000;123 Pt 3:425-462.

5. Evans J, Seri S, Cavanna AE. The effects of Gilles de la Tourette syndrome and other chronic tic disorders on quality of life across the lifespan: a systematic review. European child \& adolescent psychiatry. 2016;25(9):939-948.

6. Roessner V, Plessen KJ, Rothenberger A, et al. European clinical guidelines for Tourette syndrome and other tic disorders. Part II: pharmacological treatment. European child \& adolescent psychiatry. 2011;20(4):173-196.

7. Verdellen C, van de Griendt J, Hartmann A, Murphy T, Group EG. European clinical guidelines for Tourette syndrome and other tic disorders. Part III: behavioural and psychosocial interventions. European child \& adolescent psychiatry. 2011;20(4):197-207.

8. Muller-Vahl KR, Roessner V, European Society for the Study of Tourette S. Treatment of tics in patients with Tourette syndrome: recommendations according to the European Society for the Study of Tourette Syndrome. Movement disorders. 2011;26(13):2447; author reply 2448.

9. Ackermans L, Duits A, Temel Y, et al. Long-term outcome of thalamic deep brain stimulation in two patients with Tourette syndrome. Journal of neurology, neurosurgery, and psychiatry. 2010;81(10):10681072.

10. Ackermans L, Duits A, van der Linden C, et al. Double-blind clinical trial of thalamic stimulation in patients with Tourette syndrome. Brain. 2011;134(Pt 3):832-844.

11. Smeets AY, Duits AA, Leentjens AF, et al. Thalamic Deep Brain Stimulation for Refractory Tourette Syndrome: Clinical Evidence for Increasing Disbalance of Therapeutic Effects and Side Effects at LongTerm Follow-Up. Neuromodulation. 2017.

12. Smeets $A Y$, Duits AA, Plantinga BR, et al. Deep Brain Stimulation of the internal globus pallidus in refractory Tourette Syndrome. Clinical Neurology and Neurosurgery. 2016;142:54-59.

13. Vandewalle V, van der Linden C, Groenewegen HJ, Caemaert J. Stereotactic treatment of Gilles de la Tourette syndrome by high frequency stimulation of thalamus. Lancet. 1999;353(9154):724.

14. Bell E, Maxwell B, McAndrews MP, Sadikot A, Racine E. Deep brain stimulation and ethics: perspectives from a multisite qualitative study of Canadian neurosurgical centers. World neurosurgery. 2011;76(6):537-547.

15. Grant RA, Halpern CH, Baltuch GH, O'Reardon JP, Caplan A. Ethical considerations in deep brain stimulation for psychiatric illness. Journal of clinical neuroscience. 2014;21(1):1-5.

16. Maslen H, Pugh J, Savulescu J. The Ethics of Deep Brain Stimulation for the Treatment of Anorexia Nervosa. Neuroethics. 2015;8(3):215-230.

17. Park RJ, Singh I, Pike AC, Tan JO. Deep Brain Stimulation in Anorexia Nervosa: Hope for the Hopeless or Exploitation of the Vulnerable? The Oxford Neuroethics Gold Standard Framework. Frontiers in Psychiatry. 2017;8:44.

18. Siegel AM, Barrett MS, Bhati MT. Deep Brain Stimulation for Alzheimer's Disease: Ethical Challenges for Clinical Research. Journal of Alzheimers Disease. 2017;56(2):429-439.

19. Mink JW, Walkup J, Frey KA, et al. Patient selection and assessment recommendations for deep brain stimulation in Tourette syndrome. Movement disorders. 2006;21(11):1831-1838. 
20. Hauseux PA, Cyprien F, Cif L, et al. Long-term follow-up of pallidal Deep Brain Stimulation in teenagers with refractory Tourette syndrome and comorbid psychiatric disorders: About three cases. Journal of the European Paediatric Neurology Society. 2016.

21. Savica R, Stead M, Mack KJ, Lee KH, Klassen BT. Deep brain stimulation in tourette syndrome: a description of 3 patients with excellent outcome. Mayo Clinic proceedings. 2012;87(1):59-62.

22. Huasen B, McCreary R, Evans J, Potter G, Silverdale M. Cervical myelopathy secondary to Tourette's syndrome managed by urgent deep brain stimulation. Movement disorders. 2014;29(4):452-453.

23. Jankovic J, Kurlan R. Tourette syndrome: evolving concepts. Movement disorders. 2011;26(6):1149-1156.

24. Schrock LE, Mink JW, Woods DW, et al. Tourette syndrome deep brain stimulation: A review and updated recommendations. Movement disorders. 2014.

25. Young people's perceptions of health and health care--World Health Organization (WHO) special session. Adolescents in our society. Journal of Adolescent Health. 1992;13(5):420-436.

26. Dehne KL, Riedner G. Adolescence--a dynamic concept. Reprod Health Matters. 2001;9(17):11-15.

27. Smetana JG, Campione-Barr N, Metzger A. Adolescent development in interpersonal and societal contexts. Annual Review of Psychology. 2006;57:255-284.

28. Leckman JF, Riddle MA, Hardin MT, et al. The Yale Global Tic Severity Scale: initial testing of a clinicianrated scale of tic severity. Journal of the American Academy of Child and Adolescent Psychiatry. 1989;28(4):566-573.

29. Baldermann JC, Schuller T, Huys D, et al. Deep Brain Stimulation for Tourette-Syndrome: A Systematic Review and Meta-Analysis. Brain Stimulation. 2016;9(2):296-304.

30. Idris Z, Ghani AR, Mar W, et al. Intracerebral haematomas after deep brain stimulation surgery in a patient with Tourette syndrome and low factor XIIIA activity. Journal of clinical neuroscience. 2010;17(10):1343-1344.

31. Kaido T, Otsuki T, Kaneko Y, Takahashi A, Omori M, Okamoto T. Deep brain stimulation for Tourette syndrome: a prospective pilot study in Japan. Neuromodulation. 2011;14(2):123-128; discussion 129.

32. Pullen SJ, Wall CA, Lee KH, Stead SM, Klassen BT, Brown TM. Neuropsychiatric Outcome of an Adolescent Who Received Deep Brain Stimulation for Tourette's Syndrome. Case Report Neurological Medicine. 2011;2011:209467.

33. Vernaleken I, Kuhn J, Lenartz D, et al. Bithalamical deep brain stimulation in tourette syndrome is associated with reduction in dopaminergic transmission. Biological psychiatry. 2009;66(10):e15-17.

34. Dueck A, Wolters A, Wunsch K, et al. Deep brain stimulation of globus pallidus internus in a 16-year-old boy with severe tourette syndrome and mental retardation. Neuropediatrics. 2009;40(5):239-242.

35. Shahed J, Poysky J, Kenney C, Simpson R, Jankovic J. GPi deep brain stimulation for Tourette syndrome improves tics and psychiatric comorbidities. Neurology. 2007;68(2):159-160.

36. Hwynn N, Tagliati M, Alterman RL, et al. Improvement of both dystonia and tics with $60 \mathrm{~Hz}$ pallidal deep brain stimulation. The International journal of neuroscience. 2012;122(9):519-522.

37. Duits A, Ackermans L, Cath D, Visser-Vandewalle V. Unfavourable outcome of deep brain stimulation in a Tourette patient with severe comorbidity. European child \& adolescent psychiatry. 2012;21(9):529-531.

38. Dong S, Zhuang P, Zhang XH, Li JY, Li YJ. Unilateral deep brain stimulation of the right globus pallidus internus in patients with Tourette's syndrome: two cases with outcomes after 1 year and a brief review of the literature. The Journal of International Medical Research. 2012;40(5):2021-2028.

39. Massano J, Sousa C, Foltynie T, Zrinzo L, Hariz M, Vaz R. Successful pallidal deep brain stimulation in 15year-old with Tourette syndrome: 2-year follow-up. Journal of neurology. 2013.

40. Zekaj E, Saleh C, Porta M, Servello D. Temporary deep brain stimulation in Gilles de la Tourette syndrome: A feasible approach? Surgical neurology international. 2015;6:122.

41. Maciunas RJ, Maddux BN, Riley DE, et al. Prospective randomized double-blind trial of bilateral thalamic deep brain stimulation in adults with Tourette syndrome. Journal of neurosurgery. 2007;107(5):10041014.

42. Servello D, Porta M, Sassi M, Brambilla A, Robertson MM. Deep brain stimulation in 18 patients with severe Gilles de la Tourette syndrome refractory to treatment: the surgery and stimulation. Journal of neurology, neurosurgery, and psychiatry. 2008;79(2):136-142. 
43. Porta $M$, Brambilla $A$, Cavanna $A E$, et al. Thalamic deep brain stimulation for treatment-refractory Tourette syndrome: two-year outcome. Neurology. 2009;73(17):1375-1380.

44. Marceglia S, Servello D, Foffani G, et al. Thalamic single-unit and local field potential activity in Tourette syndrome. Movement disorders. 2010;25(3):300-308.

45. Martinez-Fernandez R, Zrinzo L, Aviles-Olmos I, et al. Deep brain stimulation for Gilles de la Tourette syndrome: a case series targeting subregions of the globus pallidus internus. Movement disorders. 2011;26(10):1922-1930.

46. Cannon E, Silburn P, Coyne T, O'Maley K, Crawford JD, Sachdev PS. Deep brain stimulation of anteromedial globus pallidus interna for severe Tourette's syndrome. The American journal of psychiatry. 2012;169(8):860-866.

47. Sachdev PS, Mohan A, Cannon E, et al. Deep brain stimulation of the antero-medial globus pallidus interna for Tourette syndrome. PloS one. 2014;9(8):e104926.

48. Kefalopoulou Z, Zrinzo L, Jahanshahi M, et al. Bilateral globus pallidus stimulation for severe Tourette's syndrome: a double-blind, randomised crossover trial. Lancet neurology. 2015;14(6):595-605.

49. Servello D, Sassi M, Brambilla A, et al. De novo and rescue DBS leads for refractory Tourette syndrome patients with severe comorbid OCD: a multiple case report. Journal of neurology. 2009;256(9):15331539.

50. Welter ML, Houeto JL, Thobois S, et al. Anterior pallidal deep brain stimulation for Tourette's syndrome: a randomised, double-blind, controlled trial. Lancet neurology. 2017.

51. Clausen J. Ethical brain stimulation - neuroethics of deep brain stimulation in research and clinical practice. The European journal of neuroscience. 2010;32(7):1152-1162.

52. Servello D, Zekaj E, Saleh C, Zanaboni Dina C, Porta M. 16 years of Deep Brain Stimulation in Tourette's Syndrome: a critical review. Journal of neurosurgical sciences. 2016.

53. Akbarian-Tefaghi L, Zrinzo L, Foltynie T. The Use of Deep Brain Stimulation in Tourette Syndrome. Brain Sci. 2016;6(3).

54. Boviatsis EJ, Stavrinou LC, Themistocleous M, Kouyialis AT, Sakas DE. Surgical and hardware complications of deep brain stimulation. A seven-year experience and review of the literature. Acta neurochirurgica. 2010;152(12):2053-2062.

55. Leckman JF, Bloch MH, Scahill L, King RA. Tourette syndrome: the self under siege. Journal of child neurology. 2006;21(8):642-649.

56. Markova S, Nikitskaya E. Coping strategies of adolescents with deviant behaviour. International Journal of Adolescence and Youth. 2017;22(1):36-46.

57. Schermer M. Ethical issues in deep brain stimulation. Frontiers in integrative neuroscience. 2011;5:17.

58. Synofzik M, Schlaepfer TE. Stimulating personality: ethical criteria for deep brain stimulation in psychiatric patients and for enhancement purposes. Biotechnology journal. 2008;3(12):1511-1520.

59. Rizzo R, Gulisano M, Martino D, Robertson MM. Gilles de la Tourette Syndrome, Depression, Depressive Illness, and Correlates in a Child and Adolescent Population. Journal of child and adolescent psychopharmacology. 2017;27(3):243-249.

60. Deeb W, Rossi PJ, Porta M, et al. The International Deep Brain Stimulation Registry and Database for Gilles de la Tourette Syndrome: How Does It Work? Frontiers in Neuroscience. 2016;10:170. 


\section{Chapter 8}

\section{Discussion}



In this thesis we have addressed questions about the role of the basal ganglia (BG) and the connected cortico-basal-ganglia-thalamocortical (CBGT) pathways in the underlying pathophysiology of TS, which is still matter of considerable debate. Knowledge about the basic functional anatomy of these pathways is essential in understanding the mechanisms and effects of Deep Brain Stimulation (DBS) in TS patients. The main goal of DBS is to restore the imbalance present in the BG of TS patients and to relieve symptoms. After many years of thalamic stimulation, we now tend to favour stimulation of the anterior internal globus pallidus (GPi), since especially the long-term effects appear to be more promising than those for thalamic stimulation.

\section{THE UNDERLYING PATHOPHYSIOLOGY OF TOURETTE SYNDROME}

\section{Neural activity in the basal ganglia of Tourette Syndrome patients}

Recording electrophysiological activity from TS patients during DBS surgery provides a unique opportunity to directly characterize the neuronal activity underlying the disorder. Extracellular neuronal recordings performed during pallidotomy surgery revealed a decreased overall firing-rate in the posterior/motor GPi of TS patients, complemented by phasic firing-rate changes around the time of the tic ${ }^{1}$. We were the first to perform these electrophysiology measures during DBS of the anterior/limbic GPi in TS patients. We found the same baseline firing-rate decrease in the anterior GPi as previously observed in the posterior $\mathrm{GPi}^{1}$, which is not reported in Parkinson patients ${ }^{2}$ or healthy non-human primates ${ }^{3}$. We also found tic dependent transient rate changes in the activity of the individual neurons of the anterior GPi, which is in line with previous measures of the posterior GPi in TS patients ${ }^{1}$. Interestingly, we also observed reduced firing rates in the anterior GPe, comparable to those previously seen in awake patients suffering from other hyperkinetic disorders such as dystonia ${ }^{4-7}$ and hemiballismus ${ }^{8}$. These results point to tonic and phasic modulations of the BG output as a key mechanism underlying the abnormal state of TS and the expression of tics, respectively. Moreover, it clearly demonstrates the involvement of the limbic pathway in the underlying pathophysiology of TS. Evidence points out that reduced inhibition in the striatum seems to play a significant role in the underlying pathophysiology of TS ${ }^{9-11}$. Therefore, we do not view these abnormal BG output signals as the cause of TS, but as a consequence of an imbalance higher in the CBGT circuit. An imbalance in for example the primary input structure of the $B G$, the striatum, can lead to alterations throughout the whole CBGT circuit. These electrophysiological studies in the acute phase of the intervention suffer from two major flaws. First, they are potentially confounded by a notable microlesion effect, which can induce biochemical responses in neurons and persist up to three months postoperatively. Second, they do not explain the underlying neurophysiological dynamics that support the pathology and how these are altered 
because of therapy. Such information must come from chronic recordings from indwelling electrodes ${ }^{12}$. Only one study has performed chronic local field potential (LFP) recordings from implanted thalamic electrodes and correlated these findings with the symptoms. Their results suggest that dynamic changes in gamma band power are relevant to the pathophysiology underlying TS and that the therapeutic effect of DBS may be the functional restoration of effective communication, since gamma band activity is characteristic of normal thalamic information processing ${ }^{13}$.

\section{Models of basal ganglia dysfunction in relation to Tourette Syndrome}

Are our results of neuronal activity in the anterior GPi and GPe in TS patients in line with the existing functional models of the BG that have suggested potential mechanisms for hyperkinetic movement disorders? The 'box and arrow' model defines the CBGT pathway as a feedback loop with two main competing elements: a positive feedback loop mediated by the direct pathway and a negative feedback loop mediated by the indirect pathway. Thus, hyperkinetic disorders like TS are associated with increased activity of the direct pathway and reduced activity of the indirect pathway, resulting in a reduction of GPi activity, which subsequently leads to disinhibition of the thalamus and overactivation of the cortex ${ }^{14-16}$. Our results support this assumption in that the GPi neurons in TS patients displayed reduced baseline activity. However, the GPe baseline activity was also reduced, contradicting the current perception of the indirect pathway involvement in hyperkinetic disorders. Interestingly, this co-modulation of both segments of the globus pallidus is not unique to TS, but has also been observed in other hyperkinetic disorders such as dystonia and hemiballismus ${ }^{6-8}$. The reduced activity in both segments of the globus pallidus can be explained by later theoretical models ${ }^{16,17}$ and experimental data from animal models ${ }^{3,18}$. The 'action selection' model suggests that a subset of excited striatal neurons leads to the release of a selected action, while inhibiting all other potential actions. In this model, hyperkinetic symptoms originate from abnormal focal activation of striatal neurons which inhibit the activity of a subpopulation of GPi neurons ${ }^{16,17,19}$. In TS, this disinhibition, affecting striatal projection neurons contributing to both the direct and indirect pathways, is thus expected to exercise increased inhibition on both the GPe and GPi driving their firing rates down, in line with our findings. Increased inhibition of the GPi leads to disinhibition of its thalamo-cortical targets and this in turn can lead to repeated stereotyped unwanted movements like tics. Next to these tonic baseline firing-rate decreases in the anterior GPi and GPe, we found a mixture of responses in both nuclei around the time of the tic, roughly equally distributed between increases and decreases. This cannot be explained by applying the above-mentioned models of BG functioning.

In summary, this points to a tonic and phasic modulation of the basal-ganglia output as a key mechanism underlying the abnormal state of TS and the expression of individual tics, respectively. Moreover, it shows that the intrinsic circuitry of the BG is 
far more complex and not all the electrophysiological findings can be explained by the existing models of BG dysfunction.

\section{Towards new models of basal ganglia function/dysfunction}

Our electrophysiology recordings highlight the differences between TS and other hyperkinetic movement disorders. The departure of our findings from the classic BG models of hyperkinetic disorders thus calls for the development of novel concepts and models of the BG, which can capture the heterogeneous nature of the different disorders as well as the different neurons within each nucleus. The emerging evidence that TS involves CBGT dysfunction in the motor and limbic domains emphasizes that further investigation of the basic neurobiology of the BG is likely to be indispensable for improved understanding of the pathophysiology of TS. Moreover, this might have direct consequences for the DBS targets and for treating refractory TS patients.

\section{DEEP BRAIN STIMULATION IN TOURETTE SYNDROME}

\section{Target selection}

Since the first DBS surgery in a refractory TS patient was performed in $1999^{20}$, about 156 TS patients have been reported to be treated with DBS. Nine separate brain areas have been targeted so far: the medial part of the thalamus (three different areas: the centromedian nucleus, substantia periventricularis, and nucleus ventro-oralis internus (Cm-Spv-Voi) cross point ${ }^{20-36}$, the centromedian nucleus-parafascicular complex (CMPf) ${ }^{37-42}$, the dorsomedial nucleus-parafascicular complex (DM-Pf) ${ }^{43}$ ), the anterior/limbic $\mathrm{GPi}^{44-52}$, the posterior/motor $\mathrm{GPi}^{48,53-63}$, the $\mathrm{GPe}^{64}$, the nucleus accumbens (NA) ${ }^{65-68}$, the anterior limb of the internal capsule (ALIC) ${ }^{65-67,69}$, and the subthalamic nucleus $\left(\right.$ STN ${ }^{70}$. Moreover, studies targetting multiple brain areas separate or at the same time have been performed ${ }^{53,71-80}$. More than $10 \%$ of these surgeries have been performed in our centre during the past 18 years ${ }^{20-23,25,34,35,51,71,81,82}$, of which we have targeted the medial thalamus in nine patients ( $\mathrm{Cm}$-Spv-Voi cross point) and the anterior GPi in eight patients. Target selection is a topic still under debate due to the complex clinical profile presented by TS patients ${ }^{12}$. The thalamus and the GPi have been targeted the most. These are key structures within the CBGT pathways and by influencing them through high frequency stimulation, the signalling might be adapted and the imbalance present in the BG of TS patients restored in order to relieve symptoms. 


\section{Patient selection}

Apart from target selection, another relevant question is which patients should be selected for DBS. TS patients considered for DBS should comprise only very severe, intractable cases since it is a last resort and experimental treatment. In 2006, the DutchFlemish Tourette Surgery Study Group has established guidelines for DBS in TS ${ }^{22}$, comparable to the guidelines from the Tourette Syndrome Association (TSA) ${ }^{83}$. We have followed the recommended in- and exclusion criteria in all our patients to ensure adequate selection of DBS candidates. In 2014, the TSA updated the recommendations for patient's selection and assessment based on a critical review of the existing literature ${ }^{83}$. A major change in these new recommendations is that the age limit has been adjusted from a minimum age of 25 years towards a multidisciplinary evaluation without setting a firm age limit. As such younger patients have been treated with DBS. This gives rise to discussion about ethical dilemmas, specific problems encountered in this age group and effect differences between adolescent and adult patients, which we will further address later in this chapter.

\section{Effects of Deep Brain Stimulation}

A meta-analysis published in 2016 by Baldermann et all ${ }^{84}$ included 156 cases of DBS in refractory TS patients. Seventy-eight cases targeted the medial thalamus, 44 the anterior GPi, 20 the posterior GPi and 9 the NA/ALIC. Highly significant changes were found after thalamic DBS (mean Yale Global Tic Severity Scale (YGTSS) improvement of 53\%). Comparable results were seen for the posterior GPi (57\%) and the anterior GPi (52\%). Changes after DBS of the ALIC/NA showed less improvement (44\%). Regarding comorbid symptoms, the data revealed a median reduction of $31 \%$ on the Yale-Brown Obsessive-Compulsive Scale (Y-BOCS) and subgroup analysis did not show significant differences between targets.

\section{Thalamic Deep Brain Stimulation}

The first TS patient who underwent thalamic DBS in our centre showed a tic reduction of $90 \%$ after 12 months ${ }^{20}$. In 2003, we reported on three patients showing a $72-90 \%$ improvement in tics eight months to five years post-operative ${ }^{35}$. In two of these three patients, Obsessive-Compulsive behaviour $(\mathrm{OCb})$ and self-injurious behaviour (SIB) were no longer present. Long-term evaluation in two patients at six and ten years postoperative demonstrated continued benefit of tic control (78\% and 92\%) ${ }^{71}$. In a larger series of 18 patients, performed by Servello et al ${ }^{33}$, who were followed up between three and 18 months, YGTSS improvement varied between $24 \%$ and $79 \%$ with a concurrent improvement in comorbidities. Follow-up of the same patients group ${ }^{75}$ showed a mean YGTSS improvement of $73 \%$ and a mean Y-BOCS improvement of $42 \%$ 
five to six years after surgery. These and other open-label studies strongly indicate that thalamic DBS may have beneficial effects in TS for tics as well as comorbid behavioural disorders $21,24,25,27-29,36-38,41,71,74,76,80$.

The results of two randomized controlled trials (RCT's) showed a more variable response. In 2007, Macuinas et al ${ }^{30}$ performed a prospective double-blind RCT targeting the thalamus ( $\mathrm{Cm}$-Pf complex) in five patients. They were randomized and evaluated in one of four states of unilateral or bilateral stimulation (both stimulators ON, left ON-right OFF, right ON-left OFF, both OFF) for one week each. In the randomized phase of the trial, a statistically significant reduction in tics was observed in the bilateral ON state. For three months open label stimulation three of the five patients responded with a $50 \%$ average reduction of tics. Measures of $\mathrm{OCb}$, depression and anxiety showed a trend towards improvement, with a $45 \%$ improvement on the $\mathrm{Y}$ BOCS. A double-blind crossover RCT performed in our centre by Ackermans et al ${ }^{22}$ targeted the Cm-Spv-Voi cross point of the thalamus in six TS patients who were randomly assigned to three months ON stimulation and three months OFF stimulation or vice versa. At a group level improvement in tic control during ON vs. OFF stimulation was 39\% and 3\%, respectively. After one-year open label follow-up, these levels were maintained at $49 \%$ on the YGTSS. Secondary outcome measures included associated behavioural disorders and mood and they did not show any effect at a group level, either between ON and OFF stimulation or between pre-operative and one-year postoperative.

We published our long-term follow-up data of these six patients ${ }^{22}$, together with a seventh patient ${ }^{21}$ (follow-up between 24 and 78 months) and found that in only one patient the DBS is still active. In three patients the target has been changed to the anterior GPi due to side effects (e.g. reduced level of energy, sleeping disorders, gaze disturbances and alteration of sexual function). Three patients were not satisfied with the results of the DBS and decided to switch off their stimulator. Two of them showed only minor improvement during stimulation, whereas the third patient had a beneficial effect but suffered from side effects. These results are in line with the long-term followup results of Mothlagh et al ${ }^{74}$, with an improvement of more than $50 \%$ in only three out of eight cases versus three out of seven cases in our sample. However, other groups did not experience more negative long-term results with thalamic DBS and did not report these or other severe side effects in their patients ${ }^{75,80}$.

\section{Pallidal Deep Brain Stimulation}

The GPi is divided in a posterolateral part, the motor area (posterior GPi), and an anteromedial part, the limbic area (anterior GPi). There has been much debate as to which sub region might respond best to DBS in TS patients. We have decided to target the anterior GPi based on pathophysiological considerations in which the limbic circuit seems to be of major importance in TS and based on positive results of other groups 
targeting this area ${ }^{84}$. Controlled data, although in a small sample, have even suggested that DBS of the anterior GPi could bring larger benefits than thalamic ${ }^{73,79}$ or posterior $\mathrm{GPi}^{48}$ stimulation. Moreover, we decided to switch our preferred target from the thalamus to the anterior GPi based on our disappointing long-term results with thalamic DBS.

Open label studies targeting the anterior GPi demonstrate a tic improvement ranging from $38 \%$ to $72 \%$ with a follow-up duration between 4 to 46 months ${ }^{44,46,48,50}$. This is in line with our results of five TS patients treated with anterior GPi DBS, showing a significant beneficial effect on tics (mean tic improvement of $70 \%$ ). We found no significant difference on comorbid behavioural disorders, mood and cognition, but there was an improvement on an individual level for $\mathrm{OCb}$. The results of anterior GPi DBS were less pronounced in two RCT's. Kefalopoulou et al ${ }^{47}$ analysed anterior GPi DBS in 15 TS patients. They compared blinded ON stimulation vs. OFF stimulation periods and showed a mean YGTSS improvement of only $15 \%$ on a group level, although with substantial variability between patients (from -3\% to 57\%). No significant effect on OCb was observed. In the open label stimulation period of 8 to 36 months patients showed a $40 \%$ improvement in tics. This significant tic improvement was maintained at long-term follow-up ${ }^{85}$. The change in OCb did not reach statistical significance, however, subgroup analysis revealed that four patients with the most severe OCb had the greatest improvement ${ }^{85}$, which is in line with our results of anterior GPi DBS ${ }^{51}$. Welter et al ${ }^{52}$ performed a double-blind RCT in which they enrolled 16 refractory TS patients who received anterior GPi DBS. Three months later they were randomly assigned to receive either active or sham stimulation for the subsequent three months. All patients then received open-label active stimulation for six months. No significant difference in YGTSS score between the beginning and the end of the three-months double-blind period between groups was found, with a mean improvement of $10 \%$ on the YGTSS for patients with active stimulation and $4 \%$ for patients with sham stimulation. YGTSS total scores for all patients in both groups combined improved with $40 \%$ at the end of the open-label period.

Data from the double-blinded period of these two RCT's targeting the anterior GPi in refractory TS patients do not support the positive effects found during their open-label phase and in other open label studies. One explanation might be that they suffer from problems concerning blinded programming, which is common in trials of DBS ${ }^{47,52}$. Adjustment of stimulation while maintaining treatment blinding can compromise the identification or use of the optimum settings. Both groups deliberately constrained the stimulation amplitudes and voltages to avoid unmasking the patient's stimulation status during this phase. Additionally, anxiety associated with uncertainty about whether stimulation was switched off or on can be an issue for some patients. During the open label phase, stimulation was further adjusted in all patients to achieve maximum symptom control. The reduction in tic severity during this period was correlated with the increase in stimulation electrical charge ${ }^{52}$. Follow-up during the blinded on and off 
phases is only three months, which seems to be insufficient to decrease tic severity for refractory TS patients. This is in line with our own experiences in which programming to reach a satisfying stimulation effect in TS patients might take months, and even up to one year. However, blinding patients for such a long period would not be acceptable or tolerated. Moreover, the inclusion period in both studies was quite long (4 years ${ }^{47}$ and 5 years ${ }^{52}$ ), and publishing the data took another 5 years in the study performed by Welter et al ${ }^{52}$.

Kefalopoulou et al ${ }^{47}$ reported that stimulation through the most dorsal contacts on the electrodes was frequently associated with optimum tic responses, possibly suggesting that stimulation of the GPe might be involved in the mechanisms of tic relieve. In their long-term follow-up analysis they investigated whether a specific anatomical site within the anterior GPi correlated with optimal clinical outcomes for tics, mood and $\mathrm{OCb}^{85}$. A region within the anterior limbic GPi, on the medial medullary lamina in the pallidum (between the border of GPi and GPe) at the level of AC-PC plane, was significantly associated with improved tics, and on an individual level with OCb. In our case series of five patients targeting the anterior $\mathrm{GPi}^{51}$, the location of the active contacts differed between patients, ranging from the anterior part of the GPi to the GPe. We could not establish a relation between the anatomical position of the active contacts and the effects of stimulation. These findings suggest that applying electrical stimulation to the region of the anterior parts of the GPe and GPi produces therapeutic effects on tics. Moreover, the stimulation effect is not limited to the area of the active contacts since the current has a range of a few millimetres around the contact, depending on the stimulation parameters. The GPe has also been shown to be a possible effective DBS target in refractory TS patients ${ }^{64,86}$.

\section{Side effects}

We have observed significant stimulation related side effects in almost all patients who underwent thalamic DBS in our centre. These side effects included reduction of energy levels, subjective gaze disturbances, and alteration of sexual function ${ }^{20,22,35}$. During long-term follow-up of these patients we saw an increasing disbalance of therapeutic effects and side effects over time, often leading to either switching the stimulator off or new surgery with a different target ${ }^{34}$. Servello et al ${ }^{33}$ also reported about stimulation induced subjective vertigo, blurring of vision and upward ocular deviation after thalamic DBS, but in their sample these symptoms appeared to be transient. Other side effects reported with thalamic DBS are anxiety, mood changes and apathy. The majority of these reported side effects appeared to be transitory or resolved with adjustments of stimulation settings ${ }^{74,84}$, which is in contrast with our results. Thoughtful programming of the stimulator is very important to achieve an optimal clinical outcome. Due to natural symptom fluctuations, variability in patient's responses to treatment and expectations, adjustment in stimulation settings is often a temporary solution. In our 
experience TS patients tend to blame the stimulator for almost all negative symptoms and maybe professionals reinforce them to do so by adjusting the stimulation settings every time they report complaints. This gives patients the feeling that every negative symptom is a side effect of the stimulator, when it might be due to a completely different cause.

We observed less severe side effects with anterior GPi DBS compared to thalamic DBS. In our group of five TS patients who underwent pallidal DBS, stimulation induced side effects were mild apathy in two patients, not responsive to changes in stimulation settings, and weight loss in another patient. Of the two adolescent patients we treated with anterior GPi DBS, one experienced side effects like dyskinesia in the legs and a dejected mood with increasing voltages, complicating the programming, and eventually leading to turning off the stimulator. Moreover, side effects did not seem to increase with longer follow-up duration and did not influence the daily functioning of patients. Others found side effects such as anxiety, agitation, apathy, mood changes and dizziness during anterior GPi DBS ${ }^{47,48,50,73,79,87}$. Most of these side effects appeared to be minor or resolved with adjustments of stimulation settings. Long-term follow-up data of anterior GPi DBS are scarce since most groups started targeting the anterior GPi only from 2011.

\section{Ethical issues}

An important point of ethical debate is the fact that evidence of the efficacy of DBS for TS is limited and it is not (yet) a recognized indication for DBS treatment by the Food and Drug Administration (FDA) and the European Commission (EC). Consequently, it must be considered an experimental treatment, which implies that only patients with the most severe symptoms that have been proven resistant to conservative treatment, can be considered candidates for DBS. Patients should be informed about the relative lack of evidence and special care should be taken avoid the so-called 'therapeutic misconception' in patients. This means that patients expect evident personal benefit, but fail to realize the experimental character of the treatment and undervaluing the fact that individual treatment response is uncertain and long-term cognitive, emotional and behavioural effects are largely unknown.

Another ethical debate has evolved around the minimum age at which DBS should be considered for refractory TS patients, especially since the guidelines do not set a strict age limit anymore. Given the serious and sometimes irreparable harm that tics can cause to the social, psychological, and intellectual development of children, surgery at younger ages is increasingly considered. However, given the natural course of TS, with improvement during and after adolescence, this may lead to implantation of DBS devices that will only be used for a few years and subsequently inactivated because tics have spontaneously remitted. Also, behavioural disturbances during puberty may interfere with compliance during programming. However, it seems to be justified, 
according to the TSA guidelines, to leave traditional reticence behind and allow earlier intervention in view of the combination of acute suffering and the chance of irreparable harm. Since 2014, we have performed DBS surgery in two adolescent TS patients. Although we have experienced spontaneous waning of symptoms making the DBS not necessary anymore in one patient and behavioural disturbances that interfered with optimal programming in the other patient, we still support that there should be no strict age limit for indicating DBS and decisions should be made on individual circumstances and symptomatology. Waiting for an eventual spontaneous regression around adolescence might cause irreparable damages to the patient's future life. Treating these adolescent TS patients underlines even more the importance of a multidisciplinary approach, maintaining strict in- and exclusion criteria, adequate social support and the preparedness of a caregiver to participate in the counselling.

Ethical reflection should accompany present and future clinical practices, preferably covering not only theoretical thought but providing also insights in the views and perspective of those concerned, that is patients, family members and professionals in order to improve this treatment.

\section{New insights and future perspectives}

The general aim of this thesis was to continue the development of DBS for refractory TS patients by further exploring the effects of surgery in our own centre. We have continued reporting new cases and the follow-up of all operated TS patients during the past 18 years. Based on the available evidence, DBS of the medial thalamus and the anterior GPi seems to be a safe and effective treatment in well-selected TS patients. Both targets are comparable with regard to tic reduction with improvements around $52 \%$ on the YGTSS, and no significant differences in improvement of comorbid behavioural disorders have been reported ${ }^{84}$. After many years of thalamic stimulation, the preferred surgical target in our centre was changed to the anterior GPi, based on our long-term results with thalamic DBS showing that there is an increasing disbalance of therapeutic effects and side effects at long-term follow-up ${ }^{34}$, and on positive results of other groups targeting this area ${ }^{52,73,79}$. We found that DBS of the anterior GPi is effective in reducing tic severity, and possibly also OCb, in refractory TS patients without serious side effects ${ }^{51}$. These results are confirmed by other open label studies ${ }^{84}$, but contradicted by data from the double-blinded period of two RCT's ${ }^{47,52}$. During this blinded period, suboptimum stimulation parameters were used out of necessity to ensure blinding, which might explain the more positive results during their open-label phase. Moreover, the short duration of the blinded on and off phases seems to be insufficient to decrease tic severity. We are cautious in over interpreting our own and other open-label data, but will continue targeting the anterior GPi as first choice in future refractory TS patients. 
On the other hand, we believe that the choice for one single DBS target in TS is unfeasible due to the complexity of the syndrome and its associated comorbidities. We probably have to switch towards tailored targeting instead of trying to find one optimal target for all patients. This tailored targeting should be based on the main symptoms of a patient. Targeting the medial thalamus might resolve a range of motor and behavioural symptoms, whereas targeting the limbic system by stimulation of the anterior GPi might influence both behavioural symptoms like $\mathrm{OCb}$ and motor symptoms. Targeting the posterior GPi could be selected for patients seeking to relieve robust motor symptoms or for patients with dystonic tics. Targeting the NA and/or ALIC might address severe behavioural symptoms in a selected group of patients. The fact that DBS of limbic structures within the CBGT circuitry, such as the anterior GPi, induces improvements comparable to DBS of motor structures favours the hypothesis that modulation of the neuronal activity within the limbic CBGT circuit provokes tic reduction. Moreover, increasing evidence shows that motor symptoms of TS can result from abnormal motivational drives from limbic circuits. In line with these considerations, we believe there is a high need for more qualitative studies and standardized incorporation of additional outcome parameters (e.g. scales for OCb, ADHD and mood disorders), other than solely focusing on the reduction of tics. In some patients, improvement of quality of life might not only be due to tic reduction, but even more due to improvement of these comorbidities.

So far there is no established consensus on stimulation targets, stimulation settings, and long-term efficacy in DBS for TS. Until that has been achieved, DBS in TS will still be experimental and must be considered a last resort treatment that should only take place in specialized treatment centres. Further research should provide more clarity in these areas and also investigate the efficacy of DBS for TS patients over longer periods with optimal stimulation parameters and also identify potential predictors of the therapeutic response, which might help adequate selection of adolescent TS patients that would benefit most from early DBS. The development of DBS in TS proceeds slowly since it is still not approved by the EC and FDA because of the lack of evidence due to the difficulty of performing high quality research in a randomized controlled fashion, coupled with the variable effect of surgery, the large number of targets, and the overall small numbers of TS patients with DBS worldwide. The experimental character of the treatment holds up further steps towards this approval. Since patients might be more cautious in undergoing the treatment, the number of operated TS patients remains low, making it very difficult to obtain enough high-quality evidence. Large scale RCT's and multicentre trails are at present not feasible, but the importance of thorough reporting of small open-label series for our daily practice cannot be underestimated. A blinded multicentred RCT including a large group of refractory TS patients, comparing the efficacy of DBS of the medial thalamus and the anterior GPi with blinded on and off periods of at least six months and an open label phase of 10 years would be desirable. However, this is impossible to realize due to practical reasons, it would stretch over 15 
to 20 years and blinding patients for such a long period would not be tolerated. Moreover, during such trial new insights might be gained, e.g. pointing to different surgical targets or other changes in the procedure, which would be unethical to withhold from later participants. In order to overcome these structural limitations, to take steps towards larger scale analyses, and to optimally benefit from the clinical experience gathered so far, we argue that all cases of DBS performed in TS patients are registered in a central database covering a range of standardized information. The TSA has launched such an international Tourette DBS registry and database and the main goal is to uncover best practices, improve outcomes, and provide critical information to regulatory agencies ${ }^{88}$.

\section{CONCLUSION}

Despite the difficulties and uncertainties about DBS in refractory TS patients, we believe that DBS holds a promise for becoming an established treatment. Further insights should come from rigorous reporting of all available data worldwide, if possible performing DBS in a blinded and RCT fashion with longer blinded periods and follow-up durations and collecting all these data in the TSA database. We will participate in continuing the development of DBS for refractory TS patients by further exploring the effects of surgery in our own centre and by contributing to the TSA database. We believe this eventually will lead to tailored targeting of brain structures for each individual TS patient based on their main symptoms. 


\section{REFERENCES}

1. Zhuang $P$, Hallett $M$, Zhang $X$, Li J, Zhang Y, Li Y. Neuronal activity in the globus pallidus internus in patients with tics. Journal of neurology, neurosurgery, and psychiatry. 2009;80(10):1075-1081.

2. Hutchison WD, Lozano AM, Davis KD, Saint-Cyr JA, Lang AE, Dostrovsky JO. Differential neuronal activity in segments of globus pallidus in Parkinson's disease patients. Neuroreport. 1994;5(12):1533-1537.

3. McCairn KW, Bronfeld M, Belelovsky K, Bar-Gad I. The neurophysiological correlates of motor tics following focal striatal disinhibition. Brain. 2009;132(Pt 8):2125-2138.

4. Alam M, Sanghera MK, Schwabe K, et al. Globus pallidus internus neuronal activity: a comparative study of linear and non-linear features in patients with dystonia or Parkinson's disease. Journal of Neural Transmission. 2016;123(3):231-240.

5. Alam M, Schwabe K, Lutjens G, et al. Comparative characterization of single cell activity in the globus pallidus internus of patients with dystonia or Tourette syndrome. Journal of Neural Transmission. 2015;122(5):687-699.

6. Sanghera MK, Grossman RG, Kalhorn CG, Hamilton WJ, Ondo WG, Jankovic J. Basal ganglia neuronal discharge in primary and secondary dystonia in patients undergoing pallidotomy. Neurosurgery. 2003;52(6):1358-1370; discussion 1370-1353.

7. Starr PA, Rau GM, Davis V, et al. Spontaneous pallidal neuronal activity in human dystonia: comparison with Parkinson's disease and normal macaque. Journal of neurophysiology. 2005;93(6):3165-3176.

8. Vitek JL, Chockkan V, Zhang JY, et al. Neuronal activity in the basal ganglia in patients with generalized dystonia and hemiballismus. Annals of neurology. 1999;46(1):22-35.

9. Kalanithi PS, Zheng W, Kataoka Y, et al. Altered parvalbumin-positive neuron distribution in basal ganglia of individuals with Tourette syndrome. Proceedings of the National Academy of Sciences of the United States of America. 2005;102(37):13307-13312.

10. Kataoka Y, Kalanithi PS, Grantz H, et al. Decreased number of parvalbumin and cholinergic interneurons in the striatum of individuals with Tourette syndrome. The Journal of comparative neurology. 2010;518(3):277-291.

11. Peterson BS, Thomas P, Kane MJ, et al. Basal Ganglia volumes in patients with Gilles de la Tourette syndrome. Archives of general psychiatry. 2003;60(4):415-424.

12. Hashemiyoon R, Kuhn J, Visser-Vandewalle V. Putting the Pieces Together in Gilles de la Tourette Syndrome: Exploring the Link Between Clinical Observations and the Biological Basis of Dysfunction. Brain Topography. 2016.

13. Maling N, Hashemiyoon R, Foote KD, Okun MS, Sanchez JC. Increased thalamic gamma band activity correlates with symptom relief following deep brain stimulation in humans with Tourette's syndrome. PloS one. 2012;7(9):e44215.

14. Albin RL, Young AB, Penney JB. The functional anatomy of basal ganglia disorders. Trends in neurosciences. 1989;12(10):366-375.

15. DeLong MR. Primate models of movement disorders of basal ganglia origin. Trends in neurosciences. 1990;13(7):281-285.

16. Albin RL, Mink JW. Recent advances in Tourette syndrome research. Trends in neurosciences. 2006;29(3):175-182.

17. Mink JW. Basal ganglia dysfunction in Tourette's syndrome: a new hypothesis. Pediatric neurology. 2001;25(3):190-198.

18. Israelashvili M, Bar-Gad I. Corticostriatal Divergent Function in Determining the Temporal and Spatial Properties of Motor Tics. The Journal of neuroscience. 2015;35(50):16340-16351.

19. Mink JW. The basal ganglia: focused selection and inhibition of competing motor programs. Progress in neurobiology. 1996;50(4):381-425.

20. Vandewalle V, van der Linden C, Groenewegen HJ, Caemaert J. Stereotactic treatment of Gilles de la Tourette syndrome by high frequency stimulation of thalamus. Lancet. 1999;353(9154):724. 
21. Ackermans $L$, Duits $A$, Temel $Y$, et al. Long-term outcome of thalamic deep brain stimulation in two patients with Tourette syndrome. Journal of neurology, neurosurgery, and psychiatry. 2010;81(10):1068-1072.

22. Ackermans $L$, Duits $A$, van der Linden $C$, et al. Double-blind clinical trial of thalamic stimulation in patients with Tourette syndrome. Brain. 2011;134(Pt 3):832-844.

23. Ackermans L, Temel Y, Bauer NJ, Visser-Vandewalle V, Dutch-Flemish Tourette Surgery Study G. Vertical gaze palsy after thalamic stimulation for Tourette syndrome: case report. Neurosurgery. 2007;61(5):E1100; discussion E1100.

24. Bajwa RJ, de Lotbiniere AJ, King RA, et al. Deep brain stimulation in Tourette's syndrome. Movement disorders. 2007;22(9):1346-1350.

25. Duits A, Ackermans L, Cath D, Visser-Vandewalle V. Unfavourable outcome of deep brain stimulation in a Tourette patient with severe comorbidity. European child \& adolescent psychiatry. 2012;21(9):529-531.

26. Huys D, Bartsch C, Koester P, et al. Motor Improvement and Emotional Stabilization in Patients With Tourette Syndrome After Deep Brain Stimulation of the Ventral Anterior and Ventrolateral Motor Part of the Thalamus. Biological psychiatry. 2016;79(5):392-401.

27. Idris Z, Ghani AR, Mar W, et al. Intracerebral haematomas after deep brain stimulation surgery in a patient with Tourette syndrome and low factor XIIIA activity. Journal of clinical neuroscience. 2010;17(10):1343-1344.

28. Kaido T, Otsuki T, Kaneko Y, Takahashi A, Omori M, Okamoto T. Deep brain stimulation for Tourette syndrome: a prospective pilot study in Japan. Neuromodulation. 2011;14(2):123-128; discussion 129.

29. Kuhn J, Bartsch C, Lenartz D, et al. Clinical effectiveness of unilateral deep brain stimulation in Tourette syndrome. Translational Psychiatry. 2011;1:e52.

30. Maciunas RJ, Maddux BN, Riley DE, et al. Prospective randomized double-blind trial of bilateral thalamic deep brain stimulation in adults with Tourette syndrome. Journal of neurosurgery. 2007;107(5):10041014.

31. Marceglia S, Servello D, Foffani G, et al. Thalamic single-unit and local field potential activity in Tourette syndrome. Movement disorders. 2010;25(3):300-308.

32. Porta M, Brambilla A, Cavanna $A E$, et al. Thalamic deep brain stimulation for treatment-refractory Tourette syndrome: two-year outcome. Neurology. 2009;73(17):1375-1380.

33. Servello D, Porta M, Sassi M, Brambilla A, Robertson MM. Deep brain stimulation in 18 patients with severe Gilles de la Tourette syndrome refractory to treatment: the surgery and stimulation. Journal of neurology, neurosurgery, and psychiatry. 2008;79(2):136-142.

34. Smeets AY, Duits AA, Leentjens AF, et al. Thalamic Deep Brain Stimulation for Refractory Tourette Syndrome: Clinical Evidence for Increasing Disbalance of Therapeutic Effects and Side Effects at LongTerm Follow-Up. Neuromodulation. 2017.

35. Visser-Vandewalle V, Temel $Y$, Boon $\mathrm{P}$, et al. Chronic bilateral thalamic stimulation: a new therapeutic approach in intractable Tourette syndrome. Report of three cases. Journal of neurosurgery. 2003;99(6):1094-1100.

36. Zekaj E, Saleh C, Porta M, Servello D. Temporary deep brain stimulation in Gilles de la Tourette syndrome: A feasible approach? Surgical neurology international. 2015;6:122.

37. Lee MW, Au-Yeung MM, Hung KN, Wong CK. Deep brain stimulation in a Chinese Tourette's syndrome patient. Hong Kong medical journal. 2011;17(2):147-150.

38. Okun MS, Foote KD, Wu SS, et al. A trial of scheduled deep brain stimulation for Tourette syndrome: moving away from continuous deep brain stimulation paradigms. JAMA Neurology. 2013;70(1):85-94.

39. Pullen SJ, Wall CA, Lee KH, Stead SM, Klassen BT, Brown TM. Neuropsychiatric Outcome of an Adolescent Who Received Deep Brain Stimulation for Tourette's Syndrome. Case Report Neurological Medicine. 2011;2011:209467.

40. Rzesnitzek L, Wachter T, Kruger R, Gharabaghi A, Plewnia C. Suppression of extrapyramidal side effects of doxepin by thalamic deep brain stimulation for Tourette syndrome. Neurology. 2011;77(18):17081709.

41. Savica R, Stead M, Mack KJ, Lee KH, Klassen BT. Deep brain stimulation in tourette syndrome: a description of 3 patients with excellent outcome. Mayo Clinic proceedings. 2012;87(1):59-62. 
42. Testini P, Zhao CZ, Stead M, Duffy PS, Klassen BT, Lee KH. Centromedian-Parafascicular Complex Deep Brain Stimulation for Tourette Syndrome: A Retrospective Study. Mayo Clinic proceedings. 2016;91(2):218-225.

43. Vernaleken I, Kuhn J, Lenartz D, et al. Bithalamical deep brain stimulation in tourette syndrome is associated with reduction in dopaminergic transmission. Biological psychiatry. 2009;66(10):e15-17.

44. Cannon E, Silburn P, Coyne T, O'Maley K, Crawford JD, Sachdev PS. Deep brain stimulation of anteromedial globus pallidus interna for severe Tourette's syndrome. The American journal of psychiatry. 2012;169(8):860-866.

45. Gallagher CL, Garell PC, Montgomery EB, Jr. Hemi tics and deep brain stimulation. Neurology. 2006;66(3):E12.

46. Huasen B, McCreary R, Evans J, Potter G, Silverdale M. Cervical myelopathy secondary to Tourette's syndrome managed by urgent deep brain stimulation. Movement disorders. 2014;29(4):452-453.

47. Kefalopoulou Z, Zrinzo L, Jahanshahi M, et al. Bilateral globus pallidus stimulation for severe Tourette's syndrome: a double-blind, randomised crossover trial. Lancet neurology. 2015;14(6):595-605.

48. Martinez-Fernandez R, Zrinzo L, Aviles-Olmos I, et al. Deep brain stimulation for Gilles de la Tourette syndrome: a case series targeting subregions of the globus pallidus internus. Movement disorders. 2011;26(10):1922-1930.

49. Massano J, Sousa C, Foltynie T, Zrinzo L, Hariz M, Vaz R. Successful pallidal deep brain stimulation in 15year-old with Tourette syndrome: 2-year follow-up. Journal of neurology. 2013.

50. Sachdev PS, Mohan A, Cannon E, et al. Deep brain stimulation of the antero-medial globus pallidus interna for Tourette syndrome. PloS one. 2014;9(8):e104926.

51. Smeets $A Y$, Duits $A A$, Plantinga BR, et al. Deep Brain Stimulation of the internal globus pallidus in refractory Tourette Syndrome. Clinical Neurology and Neurosurgery. 2016;142:54-59.

52. Welter ML, Houeto JL, Thobois S, et al. Anterior pallidal deep brain stimulation for Tourette's syndrome: a randomised, double-blind, controlled trial. Lancet neurology. 2017.

53. C vdL, H C, Vandewalle V, G A, D R, L dW. Succesful treatment of tics with bilateral internal pallidum stimulation in a 27-year old male patient with Gilles de la Tourette syndrome. Movement disorders. 2002;17:341.

54. Dehning S, Feddersen B, Cerovecki A, Botzel K, Muller N, Mehrkens JH. Globus pallidus internus-deep brain stimulation in Tourette's syndrome: can clinical symptoms predict response? Movement disorders. 2011;26(13):2440-2441.

55. Dehning S, Leitner B, Schennach R, et al. Functional outcome and quality of life in Tourette's syndrome after deep brain stimulation of the posteroventrolateral globus pallidus internus: long-term follow-up. The world journal of biological psychiatry. 2014;15(1):66-75.

56. Dehning S, Mehrkens JH, Muller N, Botzel K. Therapy-refractory Tourette syndrome: beneficial outcome with globus pallidus internus deep brain stimulation. Movement disorders. 2008;23(9):1300-1302.

57. Diederich NJ, Kalteis K, Stamenkovic M, Pieri V, Alesch F. Efficient internal pallidal stimulation in Gilles de la Tourette syndrome: a case report. Movement disorders. 2005;20(11):1496-1499.

58. Dong S, Zhang X, Li J, Li Y. The benefits of low-frequency pallidal deep brain stimulation in a patient with Tourette syndrome. Parkinsonism \& related disorders. 2014;20(12):1438-1439.

59. Dong S, Zhuang P, Zhang XH, Li JY, Li YJ. Unilateral deep brain stimulation of the right globus pallidus internus in patients with Tourette's syndrome: two cases with outcomes after 1 year and a brief review of the literature. The Journal of International Medical Research. 2012;40(5):2021-2028.

60. Dueck A, Wolters A, Wunsch K, et al. Deep brain stimulation of globus pallidus internus in a 16-year-old boy with severe tourette syndrome and mental retardation. Neuropediatrics. 2009;40(5):239-242.

61. Hwynn N, Tagliati M, Alterman RL, et al. Improvement of both dystonia and tics with $60 \mathrm{~Hz}$ pallidal deep brain stimulation. The International journal of neuroscience. 2012;122(9):519-522.

62. Shahed J, Poysky J, Kenney C, Simpson R, Jankovic J. GPi deep brain stimulation for Tourette syndrome improves tics and psychiatric comorbidities. Neurology. 2007;68(2):159-160. 
63. Zhang XH, Li JY, Zhang YQ, Li YJ. Deep Brain Stimulation of the Globus Pallidus Internus in Patients with Intractable Tourette Syndrome: A 1-year Follow-up Study. Chinese Medical Journal. 2016;129(9):10221027.

64. Piedimonte F, Andreani JC, Piedimonte L, et al. Behavioral and motor improvement after deep brain stimulation of the globus pallidus externus in a case of Tourette's syndrome. Neuromodulation. 2013;16(1):55-58.

65. Burdick A, Foote KD, Goodman W, et al. Lack of benefit of accumbens/capsular deep brain stimulation in a patient with both tics and obsessive-compulsive disorder. Neurocase. 2010;16(4):321-330.

66. Kuhn J, Lenartz D, Mai JK, et al. Deep brain stimulation of the nucleus accumbens and the internal capsule in therapeutically refractory Tourette-syndrome. Journal of neurology. 2007;254(7):963-965.

67. Neuner I, Podoll K, Lenartz D, Sturm V, Schneider F. Deep brain stimulation in the nucleus accumbens for intractable Tourette's syndrome: follow-up report of 36 months. Biological psychiatry. 2009;65(4):e5-6.

68. Zabek M, Sobstyl M, Koziara H, Dzierzecki S. Deep brain stimulation of the right nucleus accumbens in a patient with Tourette syndrome. Case report. Neurologia i neurochirurgia polska. 2008;42(6):554-559.

69. Flaherty AW, Williams ZM, Amirnovin R, et al. Deep brain stimulation of the anterior internal capsule for the treatment of Tourette syndrome: technical case report. Neurosurgery. 2005;57(4 Suppl):E403.

70. Martinez-Torres I, Hariz MI, Zrinzo L, Foltynie T, Limousin P. Improvement of tics after subthalamic nucleus deep brain stimulation. Neurology. 2009;72(20):1787-1789.

71. Ackermans L, Temel Y, Cath D, et al. Deep brain stimulation in Tourette's syndrome: two targets? Movement disorders. 2006;21(5):709-713.

72. Hauseux PA, Cyprien F, Cif L, et al. Long-term follow-up of pallidal Deep Brain Stimulation in teenagers with refractory Tourette syndrome and comorbid psychiatric disorders: About three cases. Journal of the European Paediatric Neurology Society. 2016.

73. Houeto JL, Karachi C, Mallet L, et al. Tourette's syndrome and deep brain stimulation. Journal of neurology, neurosurgery, and psychiatry. 2005;76(7):992-995.

74. Motlagh MG, Smith ME, Landeros-Weisenberger A, et al. Lessons Learned from Open-label Deep Brain Stimulation for Tourette Syndrome: Eight Cases over 7 Years. Tremor and other hyperkinetic movements. 2013;3.

75. Porta M, Servello D, Zanaboni C, et al. Deep brain stimulation for treatment of refractory Tourette syndrome: long-term follow-up. Acta neurochirurgica. 2012;154(11):2029-2041.

76. Servello D, Sassi M, Brambilla A, Defendi S, Porta M. Long-term, post-deep brain stimulation management of a series of 36 patients affected with refractory gilles de la tourette syndrome. Neuromodulation. 2010;13(3):187-194.

77. Servello D, Sassi M, Brambilla A, et al. De novo and rescue DBS leads for refractory Tourette syndrome patients with severe comorbid OCD: a multiple case report. Journal of neurology. 2009;256(9):15331539.

78. Shields DC, Cheng ML, Flaherty AW, Gale JT, Eskandar EN. Microelectrode-guided deep brain stimulation for Tourette syndrome: within-subject comparison of different stimulation sites. Stereotactic and functional neurosurgery. 2008;86(2):87-91.

79. Welter ML, Mallet L, Houeto JL, et al. Internal pallidal and thalamic stimulation in patients with Tourette syndrome. Archives of neurology. 2008;65(7):952-957.

80. Servello D, Zekaj E, Saleh C, Lange N, Porta M. Deep Brain Stimulation in Gilles de la Tourette Syndrome: What Does the Future Hold? A Cohort of 48 Patients. Neurosurgery. 2016;78(1):91-100.

81. Israelashvili $\mathrm{M}$, Smeets $\mathrm{A}$, Bronfeld $\mathrm{M}$, et al. Tonic and phasic changes in anteromedial globus pallidus activity in Tourette syndrome. Movement disorders. 2017.

82. Temel Y, Visser-Vandewalle V, Ackermans L, Beuls EA. Thalamus and penile erection. Internal Journal of Impotence Research. 2004;16(6):505-511.

83. Schrock LE, Mink JW, Woods DW, et al. Tourette syndrome deep brain stimulation: A review and updated recommendations. Movement disorders. 2014.

84. Baldermann JC, Schuller T, Huys D, et al. Deep Brain Stimulation for Tourette-Syndrome: A Systematic Review and Meta-Analysis. Brain Stimulation. 2016;9(2):296-304. 


\section{Chapter 8}

85. Akbarian-Tefaghi L, Akram H, Johansson J, et al. Refining the Deep Brain Stimulation Target within the Limbic Globus Pallidus Internus for Tourette Syndrome. Stereotactic and functional neurosurgery. 2017;95(4):251-258.

86. Vilela Filho O, Ragazzo PC, Silva DJ, Souza JT, Oliveira PM, Ribeiro TM. Bilateral globus pallidus externus DBS for the treatment of Tourette syndrome: an ongoing prospective controlled study. Stereotactic and functional neurosurgery. 2006;85:42-43.

87. Wardell K, Kefalopoulou Z, Diczfalusy E, et al. Deep brain stimulation of the pallidum internum for Gilles de la Tourette syndrome: a patient-specific model-based simulation study of the electric field. Neuromodulation. 2015;18(2):90-96.

88. Deeb W, Rossi PJ, Porta M, et al. The International Deep Brain Stimulation Registry and Database for Gilles de la Tourette Syndrome: How Does It Work? Frontiers in Neuroscience. 2016;10:170. 


\section{Summary}

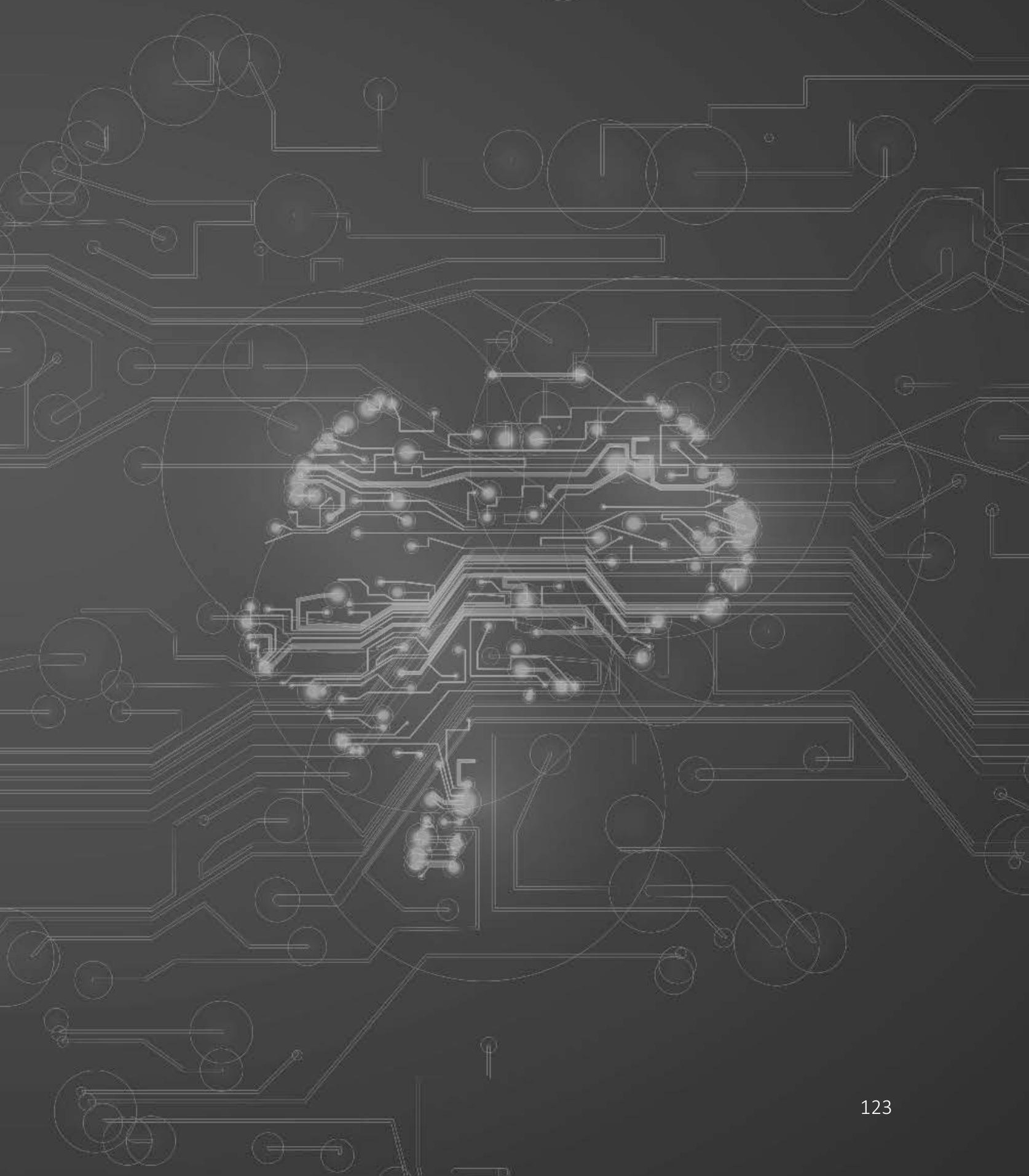



This general aim of this thesis is to continue the development of DBS for refractory TS patients by further exploring the effects of surgery in our own centre, including the relationships with the underlying pathophysiology, and both surgical and ethical issues. The main focus is to continue the follow-up of operated TS patients and TS patients with an indication for DBS, including both thalamic and anterior GPi DBS.

In chapter 2 we have investigated the role of the basal ganglia (BG) and the connected cortico-basal-ganglia-thalamocortical (CBGT) pathways in the underlying pathophysiology of TS, which is still matter of considerable debate. The BG modulate movement and behaviour through a mechanism that involves changing cortical excitability. The striatum is the main input structure of the BG and controls their output through two pathways: the direct pathway from the striatum to the output nuclei (internal globus pallidus (GPi)/ substantia nigra pars reticulata $(\mathrm{SNr})$ ), and the indirect pathway from the striatum to the $\mathrm{GPi} / \mathrm{SNr}$ through the external globus pallidus (GPe). Moreover, there is a hyperdirect pathway which bypasses the striatum and sends cortical signals directly to the subthalamic nucleus (STN) and then to the GPi/SNr. These pathways are further subdivided into functional circuits that convey motor information primarily to the posterior GPi and limbic information to the anterior GPi. Functional models of the BG have suggested potential mechanisms for hyperkinetic movement disorders like TS, which have centred on the existence of abnormalities in the motor circuits. The 'box and arrow' model depicts the global neuronal activity modulation of whole brain areas (boxes) along the CBGT pathways as a function of their connectivity (arrows). In this model, TS is explained by striatal disinhibition that leads to reduced tonic inhibition exerted by the GPi/SNr on its thalamocortical targets. The 'action selection' model accounts for the local changes in the inhibition of neuronal sub-groups within the output nuclei. In this model, TS symptoms originate from abnormal focal activation of striatal neurons, mainly due to dopaminergic dysfunction, which inhibit the activity of a subpopulation of GPi neurons. This unwanted inhibition leads to disinhibition of its thalamocortical targets and can lead to an involuntary motor command to be executed in the cortex which can lead to a tic. Electrophysiology data show that the thalamus and the GPi seem to play a significant role in the underlying pathophysiology of TS. These are key structures within the CBGT pathways and by influencing them through high frequency stimulation, the signalling can be adapted and the imbalance present in the BG of TS patients restored in order to relieve symptoms. DBS seems to exert its effect by causing functional ablation which reduces information flow through the stimulated nucleus.

In chapter 3 we report on the long-term outcome of seven refractory TS patients treated with DBS of the medial thalamus between 2001 and 2008. The target was the centromedian nucleus, substantia periventricularis and nucleus ventro-oralis internus (Cm-Spv-Voi) cross point of the thalamus. The effect on tics and side effects was evaluated with a variable follow-up duration of 12 to 78 months. Patient 1 and 2 
showed good tic improvements on the Yale Global Tic Severity Scale (YGTSS) of 81.6\% (60 months) and 50\% (36 months), respectively. However, side effects like reducing levels of energy and visual disturbances increased. In patient 1 the target was changed to the anterior GPi and patient 2 switched the stimulator permanently off. Patient 3 experiences still satisfying results with a tic improvement of $88.9 \%$ on the YGTSS (78 months). Patient 4 and 7 showed minor tic improvements of 34\% (16 months) and 9\% (60 months), respectively, and in both patients, side effects like reduced levels of energy, gaze disturbances and alteration of sexual function became more severe over time. Moreover, both patients suffered from a hardware complication; in patient 4 the entire system had to be removed due to a persisting hardware infection after a pulse generator replacement ( 6 years after surgery), and in patient 7 a hardware defect in the left electrode was detected and consequently the whole DBS system was removed ( 9 years after surgery). Since a satisfying situation with thalamic DBS had not been reached in the last years in both patients, the target was changed to the anterior GPi. Patient 5 showed a tic improvement of $27.5 \%$ on the YGTSS (12 months), was discontent with the results and went abroad for stimulation of the GPe. Patient 6 experienced several nonstimulation related side effects and developed cerebellar atrophy. Due to all these other symptoms and the lack of effect (tic improvement of $27.5 \%$ after 26 months) he turned the stimulator off and as such was lost to follow-up. Based on these results, we conclude that there seems to be an increasing disbalance of therapeutic effects and side effects at long-term follow-up after thalamic DBS, often leading to either switching the stimulator off or new surgery with a different neuro-anatomical target.

Chapter 4 reports on the results of DBS of the anterior GPi in five refractory TS patients. The target choice was based on the positive results of other groups targeting this area and our negative long-term results with thalamic DBS. This study was performed as an open label study with follow-up assessments between 12 and 38 months. Three males and two females were included with a mean age of 41.6 years (SD 9.7). The total postoperative score on the YGTSS was significantly lower than the pre-operative score $(42.2 \pm 4.8$ versus $12.8 \pm 3.8, P=0.043)$. There was also a significant reduction on the modified Rush Video-Based Tic Rating Scale (13.0 \pm 2.0 versus $7.0 \pm 1.6, P=0.041)$ and in the total number of video-rated tics $(259.6 \pm 107.3$ versus $49.6 \pm 24.8, P=0.043)$. No significant difference on the comorbid behavioural disorders, mood and cognition was found, however, there was an improvement on an individual level for ObsessiveCompulsive behaviour ( $\mathrm{OCb}$ ). The final position of the active contacts within the anterior GPi was variable in our sample and no relationship between position and stimulation effects could be established. These results suggest that DBS of the anterior GPi is effective in reducing tic severity, and possibly also OCb, in refractory TS patients without serious adverse events or side effects.

Chapter 5 describes our electrophysiology recordings during DBS surgery targeting the anterior GPi in eight awake TS patients. The neuronal activity in the anterior GPe/GPi, 
part of the limbic BG circuit, was analysed. A total of 235 single neurons were recorded and the neurons were identified as GPe $(n=77)$ or GPi $(n=158)$ neurons based on their location and trajectory history. The baseline firing rate of the globus pallidus neurons was low in TS patients. We found firing rates of $48.25 \pm 3.4$ spikes/sec and $50.9 \pm 2.6$ spikes/sec (mean \pm SEM) for GPe and GPi neurons, respectively. While the firing rates were similar between GPe and GPi neurons, the firing patterns of the neurons in each nucleus were different. Tic dependent transient rate changes were found in the activity of the individual neurons of both segments around the time of the tic (in $44.1 \%$ of GPe neurons and in $41.7 \%$ of GPi neurons). In both nuclei, half of the responding neurons displayed a decrease in activity around the time of a tic. Despite phasic tic-related changes in the firing rate of individual neurons, the mean firing rate of both nuclei did not phasically change during tic occurrence. Neither oscillatory activity of individual neurons nor correlations in their interactions were observed. Our results demonstrate the involvement of the limbic pathway in the underlying pathophysiology of TS and point to tonic and phasic modulations BG of output as a key mechanism underlying the abnormal state of the disorder and the expression of individual tics, respectively.

Chapter 6 describes a modified cement-based fixation of the DBS electrode to prevent unintended migration. This unintended electrode migration occurs in up to $10 \%$ of cases and may result in no or suboptimal therapeutic effects making a second surgery necessary. The cement-based fixation method has been our method of choice several years and we modified this method by adding anchoring screws to increase the robustness.

Chapter 7 deals with the ethical issues associated with DBS of adolescent TS patients. We discuss ethical problems encountered in two adolescent TS patients treated with DBS of the anterior GPi and systematically review the scarce literature on the topic. Three years after surgery one patient experienced a spontaneous tic reduction of more than $50 \%$ and did not require stimulation anymore. The second patient went through a period of behavioural disturbances that interfered with optimal programming, but eventually experienced a 70\% tic reduction. Only 16 surgeries in TS patients under 25 years have been reported, none of which pays attention to ethical aspects of this treatment. In adolescent TS patients, DBS carries the risk of becoming unnecessary later due to spontaneous remission. Unfortunately, since chronicity cannot be predicted, it cannot be used to indicate DBS. Moreover, reduced compliance due to behavioural disturbances that may occur in this age period can interfere with treatment. The ethical discussion about DBS in adolescent TS patients is important and should precede future practices and accompany the current practice of indication and treatment.

The general discussion in chapter 8 addresses questions about the underlying pathophysiology of TS and reflects on what has been learned thus far about thalamic and anterior GPi DBS in refractory TS patients. Recommendations for clinical practice and challenges for future research are discussed. 



\section{Samenvatting}

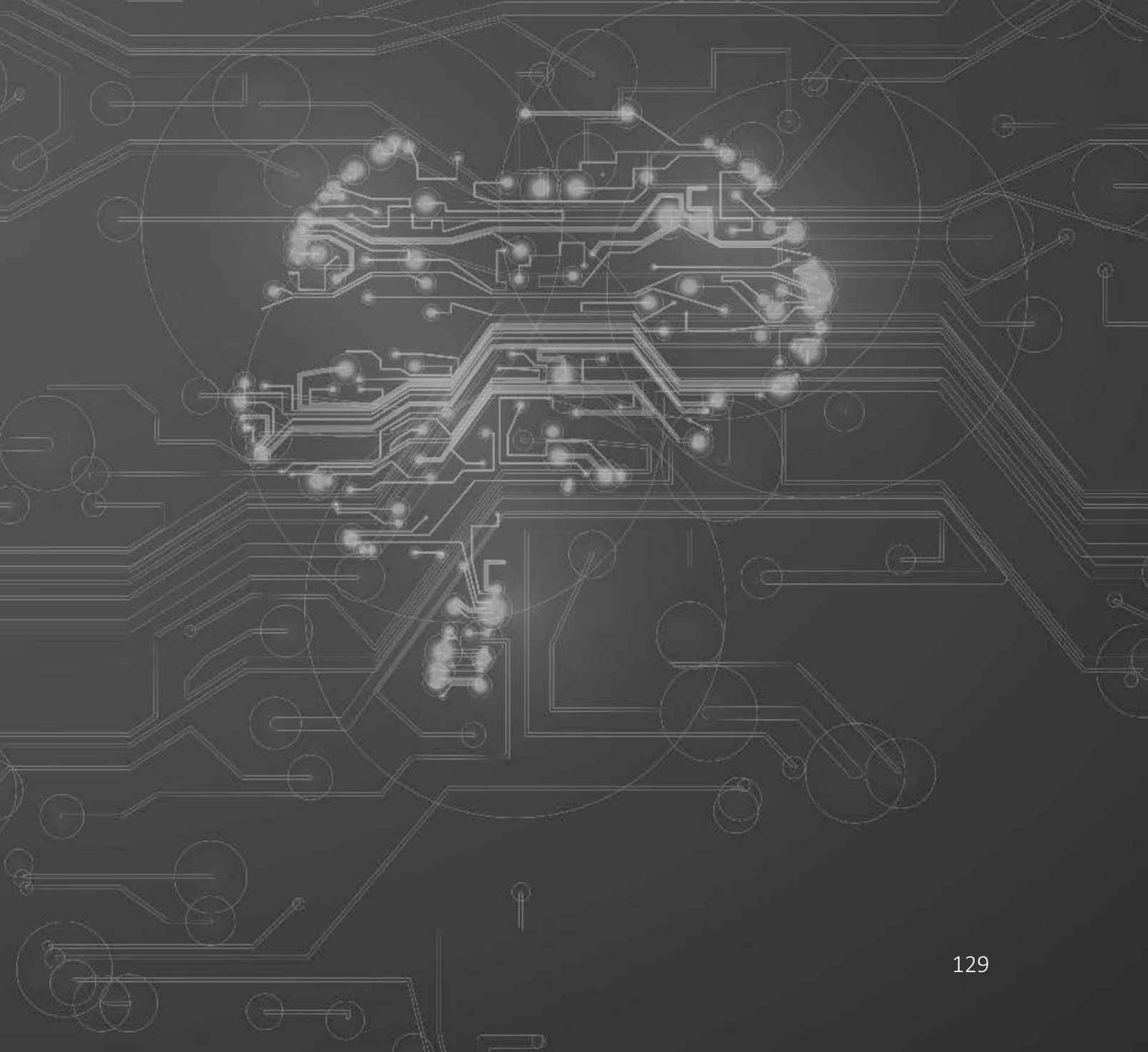



Het algemene doel van dit proefschrift is bij te dragen aan de ontwikkeling van diepe hersenstimulatie (DBS) bij patiënten met een refractaire vorm van het Syndroom van Gilles de la Tourette (TS). De focus ligt op het beschrijven van nieuwe bevindingen over de onderliggende pathofysiologie en het verkrijgen van nieuwe inzichten over de effecten van thalamische en pallidale DBS bij refractaire TS patiënten.

In hoofdstuk 2 hebben we de rol van de basale ganglia (BG), en de daarmee verbonden cortico-basale-ganglia-thalamocorticale (CBGT) circuits, onderzocht in de onderliggende pathofysiologie van TS, een onderwerp van continue discussie. De BG moduleren beweging en gedrag door het veranderen van de corticale prikkelbaarheid. Het striatum is de belangrijkste input structuur van de BG en controleert de output door middel van twee pathways: de directe pathway van het striatum naar de output kernen van de BG (globus pallidus internus (GPi)/substantia nigra pars reticulata $(\mathrm{SNr})$ ), en de indirecte pathway van het striatum naar de GPi/SNr door de globus pallidus externus (GPe). Daarnaast is er nog een hyperdirecte pathway welke corticale signalen direct naar de nucleus subthalamicus (STN) stuurt en dan naar de GPi/SNr. Deze pathways worden verder onderverdeeld in functionele pathways waarbij motorische informatie voornamelijk naar de posterieure GPi gestuurd wordt en limbische informatie naar de anterieure GPi. Functionele modellen van de BG hebben potentiele mechanismen voor hyperkinetische bewegingsstoornissen zoals TS voorgesteld, welke zich concentreren op afwijkingen binnen de motorische circuits. Het 'box and arrow' model beschrijft de globale modulatie van de neuronale activiteit van hele hersengebieden (boxes) langs de CBGT circuits als een functie van hun connectiviteit (arrows). In dit model wordt TS verklaard door disinhibitie in het striatum wat leidt tot verminderde tonische inhibitie van de GPi/SNr op de thalamocorticale doelgebieden. Het 'action selection' model beschrijft lokale veranderingen in de inhibitie van neuronale subgroepen binnen de output kernen. In dit model ontstaan de symptomen van TS vanuit abnormale focale activatie van neuronen in het striatum, met name door dopaminerge dysfunctie, welke de activiteit van een subpopulatie van GPi neuronen inhiberen. Deze ongewilde inhibitie leidt tot disinhibitie van de thalamocorticale doelgebieden en kan leiden tot een onwillekeurige motorische opdracht in de cortex en tot het ontstaan van een tic. Elektrofysiologie data tonen dat de thalamus en de GPi een significante rol spelen in de onderliggende pathofysiologie van TS. Beide zijn belangrijke structuren binnen het CBGT circuit en door ze te beïnvloeden met hoogfrequente stimulatie, kan de signalering door de BG aangepast worden. DBS lijkt zijn effect uit te voeren door functionele ablatie te veroorzaken waardoor de informatie geleiding door de gestimuleerde kern verminderd wordt. Het voornaamste doel van DBS is om de disbalans in de BG, welke aanwezig is bij TS, te herstellen en symptomen te verlichten.

In hoofdstuk 3 beschrijven we de langetermijnresultaten van zeven patiënten met een refractaire vorm van TS welke behandeld zijn middels DBS van de mediale thalamus tussen 2001 en 2008. De target was het kruispunt van centromediane nucleus - 
substantia periventricularis - nucleus ventro-oralis internus (Cm-Spv-Voi) van de thalamus. Het effect op tics en bijwerkingen werd geëvalueerd met een variabele follow-up duur van 12 tot 78 maanden. Patiënt 1 en 2 toonden een goede verbetering in tics van $81.6 \%$ (60 maanden) en 50\% (36 maanden) op de Yale Global Tic Severity Scale (YGTSS), respectievelijk. Echter, bijwerkingen zoals energieverlies en visuele stoornissen namen in de loop van de tijd toe. Bij patiënt 1 werd daarop de target aangepast middels een $2^{\mathrm{e}}$ DBS operatie naar de anterieure GPi. Bij patiënt 2 werd in overleg besloten om de stimulator definitief uit te zetten. Patiënt 3 ervaart nog steeds een goed effect van zijn DBS met een verbetering van $88.9 \%$ op de YGTSS (78 maanden). Patiënt 4 en 7 toonden een minimale verbetering van tics van respectievelijk 34\% (16 maanden) en 9\% (60 maanden). Bij beide patiënten namen bijwerkingen, waaronder energieverlies, visusstoornissen en seksuele functiestoornissen toe in de loop van de tijd. Daarnaast trad in beide gevallen een hardware complicatie op; bij patiënt 4 moest het hele DBS systeem verwijderd worden door een persisterende hardware infectie na het vervangen van de pulsgenerator (6 jaar postoperatief), en bij patiënt 7 werd een hardware defect in de linker elektrode gevonden waardoor het hele systeem verwijderd werd ( 9 jaar postoperatief). Bij beide patiënten was er de laatste jaren geen sprake meer van een acceptabel effect van de DBS en daarom werd besloten de target te wijzigen naar de anterieure GPi. Patiënt 5 toonde een verbetering van 27.5\% op de YGTSS (12 maanden) en is naar het buitenland gegaan om een DBS van de globus pallidus externus te laten verrichten. Verdere follow-upgegevens ontbreken. Patiënt 6 ontwikkelde postoperatief cerebellaire atrofie met een scala aan klachten (onder andere lethargie, dysartrie en loopstoornissen), die geen relatie hadden met de stimulatie. Daarnaast was het effect op tics minimaal (34\% verbetering na 26 maanden) en is besloten om de stimulator uit te zetten. Deze resultaten tonen dat er op de lange termijn een toenemende disbalans lijkt te zijn tussen therapeutische effecten en bijwerkingen van thalamische DBS. Dit zorgt er vaak voor dat de stimulator of uitgezet worden of dat er een nieuwe operatie plaatsvindt met een andere neuroanatomisch target.

Hoofdstuk 4 beschrijft de resultaten van DBS van de anterieure GPi in vijf patiënten met een refractaire vorm van TS. De target keuze was gebaseerd op de positieve resultaten van anderen welke dit gebied stimuleren en onze eigen negatievere lange termijn resultaten met thalamische DBS. Deze studie is uitgevoerd als een open label studie met een variabele follow-up duur van 12 tot 38 maanden. Drie mannen en twee vrouwen werden geïncludeerd met een gemiddelde leeftijd van 41.6 jaar (SD 9.7). De totale postoperatieve score op de YGTSS was significant lager dan de preoperatieve score $(42.2 \pm 4.8$ versus $12.8 \pm 3.8, P=0.043)$. Er was ook een significante verbetering op de modified Rush Video-Based Tic Rating Scale (13.0 \pm 2.0 versus 7.0 $\pm 1.6, P=0.041)$ en in het totale aantal video-gescoorde tics (259.6 \pm 107.3 versus 49.6 $\pm 24.8, P=0.043)$. Er werden geen significante verschillen gevonden in comorbide gedragsstoornissen, stemming en 
cognitie. Echter, er op een individueel level was er een verbetering in obsessiefcompulsief gedrag. De uiteindelijke positie van de actieve contacten van de definitieve elektrode binnen de anterieure GPi was variabel in onze groep en er kon geen relatie gevonden worden tussen de positie en de stimulatie effecten. Deze resultaten tonen aan dat DBS van de anterieure GPi effectief is in het verminderen van tics, en mogelijk ook obsessief-compulsief gedrag, bij refractaire TS patiënten zonder dat er sprake is van ernstige bijwerkingen.

Hoofdstuk 5 gaat over onze elektrofysiologie metingen tijdens DBS operaties van acht wakkere TS patiënten met als target de anterieure GPi. De neuronale activiteit in de anterieure GPe/GPi, onderdeel van het limbische CBGT circuit, werd geanalyseerd. In totaal werden 235 afzonderlijke neuronen geregistreerd en deze werden geïdentificeerd als GPe $(n=77)$ of GPi $(n=158)$, gebaseerd op locatie en traject geschiedenis. De basis vuursnelheid van de globus pallidus neuronen was laag in TS patiënten. We vonden vuursnelheden van $48.25 \pm 3.4$ pieken/sec en $50.9 \pm 2.6$ pieken/sec (gemiddelde \pm SEM) voor GPe neuronen en GPi neuronen, respectievelijk. Terwijl de vuursnelheden gelijk waren tussen de GPe en GPi neuronen, waren de vuurpatronen van de neuronen in de afzonderlijke kernen verschillend. Tic gerelateerde en voorbijgaande veranderingen in vuursnelheid werden gezien in de activiteit van de individuele neuronen van beide kernen rond het moment van de tic (bij $44.1 \%$ van de GPe neuronen en bij 41.7\% van de GPi neuronen). In zowel de GPe als de GPi liet de helft van deze reagerende neuronen een afname in activiteit zien rond het moment van de tic. Ondanks deze fasische tic-gerelateerde veranderingen in vuursnelheid van individuele neuronen, veranderde de gemiddelde vuursnelheid van beide kernen niet tijdens een tic. Zowel oscillaties van individuele neuronen als correlaties in hun interacties werden niet gevonden. Onze resultaten demonstreren dat het limbische circuit betrokken is bij de onderliggende pathofysiologie van TS. Daarnaast tonen ze aan dat tonische en fasische verandering van de BG output een sleutelmechanisme is in de onderliggende abnormale status van de ziekte en de expressie van individuele tics, respectievelijk.

Hoofdstuk 6 beschrijft een gemodificeerde cement-fixatie van de DBS-electrode om ongewilde migratie te voorkomen. Deze ongewilde migratie treedt op bij circa $10 \%$ van de operaties en dit kan resulteren in geen of suboptimale therapeutische effecten, waardoor een tweede operatie noodzakelijk is. We hebben de afgelopen 15 jaar gebruik gemaakt van cement-fixatie en hebben deze procedure gemodificeerd door verankeringsschroeven toe te voegen om de stevigheid van de constructie te verbeteren.

Hoofdstuk 7 gaat over ethische kwesties welke geassocieerd zijn met DBS bij adolescente TS patiënten. Wij bediscussiëren de ethische problemen welke wij tegengekomen zijn bij de behandeling van twee adolescente TS patiënten met DBS van de anterieure GPi. Daarnaast analyseren we de spaarzame literatuur over dit 
onderwerp. Drie jaar na de operatie was er bij één patiënt sprake van een spontane tic verbetering van $50 \%$ en hij had de stimulatie niet meer nodig. De tweede patiënt heeft postoperatief een periode van gedragsproblemen doorgemaakt welke interfereerden met optimale programmering, maar uiteindelijk was er sprake van $70 \%$ tic vermindering. Slechts 16 operaties in TS patiënten < 25 jaar zijn beschreven waarvan geen enkele aandacht bestaat aan de ethische aspecten van deze behandeling. Bij adolescenten bestaat er een risico dat DBS op termijn niet meer noodzakelijk is door spontane remissie van de ziekte. Helaas kan chroniciteit bij TS niet voorspeld worden en kan het dus ook niet gebruikt worden om de indicatie voor DBS te stellen. Daarnaast kan verminderde compliantie door gedragsproblemen sneller optreden in deze levensfase wat een negatieve invloed kan hebben op de behandeling. De ethische discussie rondom DBS bij adolescente TS patiënten is belangrijk en hier moet meer aandacht voor zijn bij toekomstig onderzoek.

In de algemene discussie in hoofdstuk 8 beschrijven we nieuwe ideeën over de onderliggende pathofysiologie van het TS. Daarnaast trekken we lering uit onze eigen ervaringen met DBS van de thalamus en de anterieure GPi bij TS patiënten. Adviezen voor de klinische praktijk en uitdagingen voor toekomstig onderzoek worden besproken. 


\section{Valorisation}



Tourette Syndrome (TS) is a childhood-onset neurodevelopmental disorder characterized by multiple motor and one or more vocal tics. It is frequently accompanied by comorbid behavioural disorders and they often cause even more impairment than the tics themselves. TS affects between $0.4 \%-3.8 \%$ of the general population. Mainly children between 5 and 18 years of age suffer from the disease and the prevalence in this group lies around $1 \%$. Individuals in all countries worldwide can be affected, although to different degrees. Fortunately, TS is often self-limiting around adolescence. About one third of children experiences a significant tic reduction during adolescence and another third becomes completely tic-free. Yet, still one third of children does not experience this remission, and some not only experience tic worsening during adolescence but they develop the most severe forms of TS. In those patients, TS has a devastating influence on their lives and social environments. Since there is no cure for TS, treatment aims to diminish or alleviate tics and comorbid symptoms. Despite behavioural therapy and pharmacological treatment, a small subgroup of patients fails to respond to treatment or experiences intolerable side effects. These patients are often not able to go to school or work, tend to avoid social activities, are not able to develop friendships or an affective relationship, and have a low self-esteem. For those severely affected TS patients, Deep Brain Stimulation (DBS) has emerged as a therapeutic escalation. In refractory cases of well selected TS patients, DBS of different targets within the thalamus and the basal ganglia (BG) seems to be a promising treatment. A key factor in attaining optimal results is target selection, a topic still under debate due to the complex clinical profile presented by TS patients. Nine separate brain areas have been targeted with DBS so far in TS patients.

This thesis contributes to the clinical evidence that DBS, targeting the medial thalamus and the anterior internal globus pallidus (GPi), seems to be a safe and effective treatment in strictly selected TS patients at the short-term. We found an increasing disbalance of therapeutic effects and side effects at long-term follow-up with thalamic DBS, often leading to either switching the stimulator off or new surgery with a different target. DBS of the anterior GPi was found to be effective in reducing tic severity, and possibly also Obsessive-Compulsive behaviour (OCb), without serious side effects, at both the short-term and the long-term. Because of this we think that DBS for future refractory TS patients should target the anterior GPi and not the thalamus. However, we believe that the choice for a single DBS target in TS is unfeasible given the heterogeneity of the disease. As such we need to switch towards tailored targeting instead of trying to find the optimal target for all patients. This tailored targeting should be based on the main symptoms of a patient, e.g. tics or comorbid behavioural disorders. Besides the specific target another clinical issue considers the age limit for DBS in TS. In spite of our experienced difficulties with adolescent DBS for TS, we think that there should be no strict age limit for indicating DBS and decisions should be made on individual circumstances and symptomatology. Waiting for an eventual spontaneous regression around adolescence might cause irreparable damages to the patient's future 
life. We think that ongoing ethical discussion is of major importance and should precede future practices and accompany the current practice of indication and treatment.

The development of DBS in TS proceeds slowly since it is not (yet) approved by the Food and Drug Administration (FDA) and the European Commission (EC), because of the lack of evidence due to the difficulty of performing high quality research in a randomized controlled fashion, coupled with the variable effect of surgery, the large number of targets, and the overall small numbers of TS patients with DBS worldwide. The experimental character of the treatment holds up further steps towards approval. Since patients are more cautious in undergoing the treatment and they can only be operated in a research setting, the numbers of included TS patients remain low, making it very difficult to obtain enough high-quality evidence for approval by the regulatory agencies. The various centres use different techniques and targets, making it difficult to obtain and interpret large-scale outcomes. Multicentre randomized controlled trials would theoretically be desirable, but difficult to realize due to practical reasons and would stretch over many years. Moreover, during such trial new insights might be gained, e.g. pointing to different surgical targets or other changes in the procedure, which would be unethical to withhold from later participants. In order to optimally benefit from the clinical experience, we think that all cases of DBS for TS should be entered in a central database covering a range of standardized information, initiated by the Tourette Syndrome Association (TSA). The main goal is to share data, uncover best practices, improve outcomes, and provide critical information to regulatory agencies. Despite the difficulties and uncertainties about DBS in refractory TS patients, we believe that DBS holds a promise for becoming an established treatment. We will participate in continuing the development of DBS for refractory TS patients by further exploring the effects of surgery in our own centre and by contributing to the TSA database. 


\section{Dankwoord}



Alle mensen die hebben bijgedragen aan dit proefschrift, met in het bijzonder mijn promotieteam, wil ik van harte bedanken. Zonder jullie had ik dit nooit voor elkaar kunnen krijgen.

Prof. Y Temel, beste Yasin, jij hebt mij als student de kans gegeven om onderzoek te komen doen binnen jouw team. Je visie heeft mij altijd gemotiveerd. Je begeleiding heeft mij uitgedaagd om meer te doen en beter te willen zijn. Jouw vertrouwen heeft mij doen groeien. Ik hoop dat ik tijdens de rest van mijn carrière nog veel van je zal blijven leren.

Dr. L Ackermans, beste Linda, jij hebt mij de kans gegeven om het werk van je eigen proefschrift voort te zetten. Je hebt mij het vertrouwen gegeven om jouw onderzoek verder uit te breiden en om samen nieuwe ideeën te onderzoeken. Als collega en neurochirurg ben jij mijn voorbeeld. Je oprechte interesse, eerlijkheid en steun in het schrijven van dit proefschrift en in het krijgen van een opleidingsplek zijn van onschatbare waarde geweest gedurende de afgelopen jaren.

Dr. A Duits, beste Annelien, ik ben blij dat ik met jou heb mogen samenwerken de afgelopen jaren. In de begeleiding van patiënten, het verzamelen en analyseren van data, en het schrijven en reviseren van artikelen heb je een grote rol gespeeld. Daarin heb ik ontzettend veel van je geleerd. Ik weet zeker dat patiënten bij niemand anders beter af zijn. Je bent een ontzettend goede neuropsychologe en een hele fijne collega.

Dr. A Leentjens, beste Albert, bedankt voor je begeleiding en bijdrage tijdens het doorlopen van mijn promotietraject en het schrijven van dit proefschrift. Door je snelle, goede, en volledige feedback op ieder artikel, iedere vraag, en ieder hoofdstuk, is het voor mijn gevoel vaak in een sneltreinvaart gegaan. Je bent een begeleider waar iedere promovendus jaloers op kan zijn.

Naast mijn promotieteam zijn er ook vele andere die hebben bijgedragen aan het tot stand komen van dit proefschrift. Enkele personen wil ik in het bijzonder bedanken.

Op de eerste plaats de Tourette patiënten en hun families. Zonder jullie bereidheid en openheid om iedere keer weer mee te werken aan het onderzoek en alle gegevens te delen met collega's wereldwijd, was dit proefschrift er niet geweest. Zonder deze bereidheid waren de lessen die wij van jullie behandeling hebben mogen leren, verloren gegaan. Ik ben dankbaar dat ik jullie heb leren kennen, mogen behandelen en begeleiden.

Nicole Bakker, als casemanager functionele neurochirurgie ben je nauw betrokken bij de patiëntenzorg rondom dit proefschrift. Maar je bent voor mij veel meer dan de casemanager. Met al mijn vragen kon ik bij jou terecht, je was altijd bereid om mee te denken en mee te helpen. De logistiek was onmogelijk geweest zonder jou.

Staf Neurochirurgie MUMC Maastricht/Zuyderland Heerlen, jullie hebben mij het vertrouwen gegeven om te starten met de opleiding tot neurochirurg. De 
onzekerheden en het harde werken als ANIOS, het voorzetten van mijn onderzoek naast de kliniek, de spanning rond de opleidingsplek, het is het allemaal meer dan waard geweest. Als er één ding is waar ik van overtuigd ben, is dat er voor mij geen mooier vak bestaat dan neurochirurgie. Ik ben blij dat jullie degene zijn die mij dit vak willen leren. Beste Jasper, jou wil ik van alle stafleden toch nog even noemen. Tijdens het $3^{\mathrm{e}}$ jaar van de studie geneeskunde heb je me uit de onderwijsgroep geplukt en op OK in Heerlen naast jou en Onno neergezet, een moment dat ik nooit meer zal vergeten. Daar is voor mij de liefde voor het vak neurochirurgie begonnen en daar zal ik je altijd dankbaar voor zijn. Maar wat voor mij nog veel belangrijker is geweest, is dat er iemand was die iets zag in mij, iemand die aardig en betrokken was, iemand die al mijn vragen wilde beantwoorden. Je hebt me veel wijze lessen geleerd de afgelopen jaren.

Toon, Vincent, Youssef, Roel, Felix, Tim, Lars, Karlijn, Jeroen, en Rick dankjewel voor de ruimte die jullie me hebben gegeven om dit proefschrift te schrijven. Dankjewel voor de oprechte interesse in mijn onderzoek en in mij als persoon. Dankjewel voor alle gezelligheid tijdens en na het werk. Het is ontzettend veel waard om te weten dat er anderen zijn die met dezelfde uitdagingen te maken hebben en om te weten dat er collega's zijn bij wie je terecht kunt.

Our colleagues from Israel, prof. I Bar-Gad en drs. M Israelashvili, dear Ihzar en Michal. Thank you for the great collaboration we achieved. I have learned a lot from all our Skype conversations and I am glad I got to know you.

Mijn vrienden, dank jullie wel voor alle dagen, avonden en nachten die zorgden voor de nodige ontspanning. Jullie vriendschappen zijn heel belangrijk voor mij. Ze geven mij liefde, vrolijkheid, en steun, ze geven mij energie om door te gaan.

Lieve Noortje en Anneke, we kennen elkaar al een eeuwigheid en door jaren samen in één huis te wonen, hebben we elkaar nog beter leren kennen. We hebben samen de studententijd doorlopen en zijn los van elkaar hard aan het werk om specialist te worden. Jullie nuchtere kijk op dingen en eerlijke mening zijn soms nodig om weer wat perspectief te krijgen. Jullie gezelligheid is van onschatbare waarde. Ik ben ontzettend dankbaar voor jullie vriendschap en ik ben blij dat ik jullie als paranimf aan mijn zijde mag hebben. Lieve Hester, samen met huize Teunwissmeets maakte je het Heerderweg clubje af tijdens de studententijd. Ook nu je in Utrecht woont en werkt, is de vriendschap er niet minder op geworden. Ik ben blij zo'n lieve meid als jou als vriendin te hebben. Lieve Jolien, het feit dat je aan de andere kant van de wereld woont, doet niks af aan onze vriendschap, ik ben blij jou in mijn leven te hebben. Lieve Alex, dankjewel dat je er altijd voor me bent als ik je nodig heb. Onze vriendschap is net zo vanzelfsprekend als familie, en dat maakt het des te bijzonderder. We hebben al heel veel mooie, maar ook moeilijke momenten met elkaar mogen delen. Lieve Cindy, ook jij maakt deel uit van deze vriendschap en ik ben dankbaar dat ik jou heb leren kennen. 
Mijn familie. Lieve mama en papa, mijn boekje is af. Ik kan het soms zelf nog niet helemaal geloven. Jullie staan aan de basis van alles wat ik heb mogen doen en bereiken in mijn leven. Uit de grond van mijn hart dank ik jullie voor de onvoorwaardelijke steun en liefde die ik van jullie krijg. Jullie hebben me altijd de ruimte gegeven om mijn eigen keuzes te maken. Voor advies en hulp kan ik altijd bij jullie terecht, nooit is jullie iets teveel. Lieve Sheila, je bent niet alleen mijn kleine zusje maar ook één van mijn beste vriendinnen. Ik ben ontzettend blij dat ik met jou ben opgegroeid, dat we samen volwassen zijn geworden, en dat we zoveel mooie momenten hebben gedeeld. We zijn gezegend om onderdeel uit te maken van zo'n lief en warm gezin. Ik ben ontzettend trots op je en weet zeker dat je een hele mooie toekomst tegemoet gaat.

Lieve oma Smeets, u bent altijd blij als ik op bezoek kom, geïnteresseerd en betrokken, ik hoop dat ik nog jaren van u aanwezigheid mag genieten. Lieve opa Smeets, u bent er helaas niet meer om mijn verdediging mee te maken, iets waar u zo trots op geweest zou zijn. Ik wil u bedanken voor de oneindige interesse in mij en in mijn proefschrift, altijd vroeg u erna, alles wilde $u$ zien en lezen.

Lieve schoonfamilie, George en Annemie, Emma en Dim, dankjewel voor het tweede thuis wat ik erbij gekregen heb.

Lieve Rutger, zonder jou was dit proefschrift nooit afgekomen. Je bent mijn grote steun en toeverlaat. Als ik het even niet meer zag zitten, kwam jij met je wijze woorden om alles weer wat te relativeren. Je hebt me altijd gestimuleerd om door te gaan, om beter te willen zijn, om neurochirurg te worden en mijn proefschrift af te ronden. Nooit was iets je teveel of te lastig, hoe drukker ik het had, hoe meer jij thuis overnam. Je bent trots op me en daardoor wil ik het alleen maar beter doen. Je haalt het beste in mij naar boven en daar ben ik je eeuwig dankbaar voor. 



\section{Curriculum Vitae}

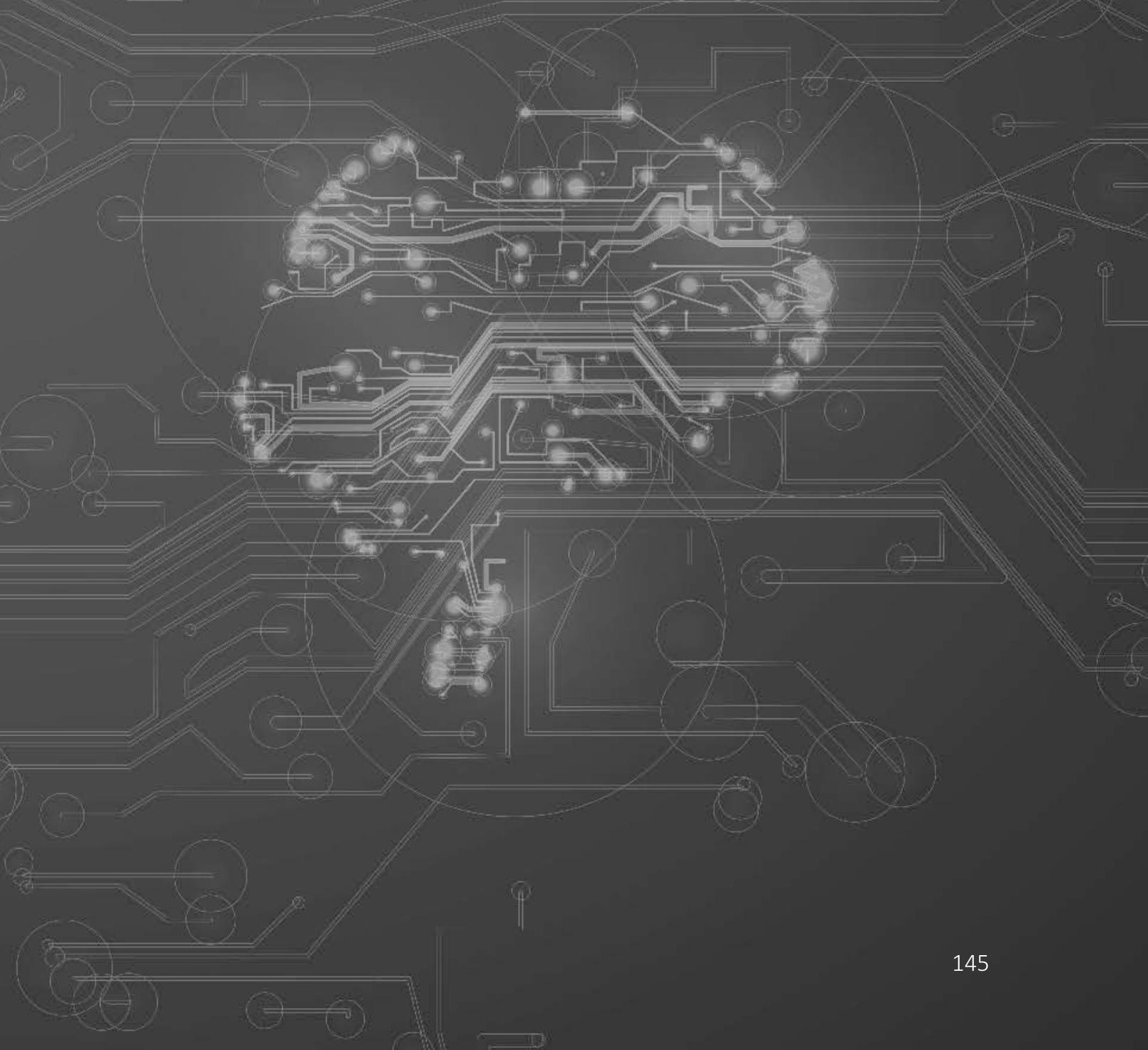



Anouk (Yvonne Jacqueline Maria) Smeets was born on March 24 ${ }^{\text {th }}, 1989$ in Heerlen, the Netherlands. She attended secondary school for six years at the 'Bernardinuscollege' in Heerlen. In 2007 she started to study medicine at the Faculty of Health, Medicine and Life Sciences at the Maastricht University. At the end of her medical studies she completed her research internship about Deep Brain Stimulation in refractory Tourette Syndrome and the beginning of this thesis was set. In December 2013 she received her medical doctor degree and in February 2014 she started working as a resident at the Neurosurgery department at the Maastricht University Medical Centre. In October 2015 she started her specialist training in Neurosurgery at the same department. During her residency she continued and completed the research described in this thesis under supervision of Prof. Temel, Dr. Ackermans, Dr. Duits, and Dr. Leentjens.

Anouk (Yvonne Jacqueline Maria) Smeets werd op 24 maart 1989 geboren in Heerlen. Ze doorliep het gymnasium aan de middelbare school het 'Bernardinuscollege' in Heerlen. In 2007 begon ze met de studie Geneeskunde aan de Faculty of Health, Medicine and Life Sciences van de Universiteit Maastricht. Gedurende het laatste jaar van haar studie bestond haar wetenschapsparticipatie uit onderzoek naar diepe hersenstimulatie bij het syndroom van Gilles de la Tourette, dit was eveneens het begin van deze thesis. In december 2013 behaalde ze haar artsendiploma en in februari 2014 begon ze met werken als arts-assistent bij de afdeling Neurochirurgie in het Maastricht Universitair Medisch Centrum. In oktober 2015 werd ze aldaar aangenomen voor de opleiding tot neurochirurg. Tijdens deze opleiding werd, onder begeleiding van Prof. Temel, Dr. Ackermans, Dr. Duits, en Dr. Leentjens het proefschrift geschreven dat nu voor u ligt. 



\section{List of publications}





\section{ARTICLES}

Smeets AY, Ackermans L, Oosterloo M, Kuijf ML, van Overbeeke JJ, Visser-Vandewalle V, Temel Y. Modified cement-based fixation of the deep brain stimulation electrode. Stereotactic and functional neurosurgery. 2015;93(1):67.

Smeets AY, Duits AA, Plantinga BR, Leentjens AF, Oosterloo M, Visser-Vandewalle V, Temel Y, Ackermans L. Deep Brain Stimulation of the internal globus pallidus in refractory Tourette Syndrome. Clinical Neurology and Neurosurgery. 2016;142:54-59.

Smeets AY, Duits AA, Leentjens AF, Schruers K, van Kranen-Mastenbroek V, VisserVandewalle V, Temel Y, Ackermans L. Thalamic Deep Brain Stimulation for Refractory Tourette Syndrome: Clinical Evidence for Increasing Disbalance of Therapeutic Effects and Side Effects at Long Term Follow-Up. Neuromodulation. 2017.

Israelashvili M, Smeets AY, Bronfeld M, Zeef DH, Leentjens AF, van Kranen-Mastenbroek V, Janssen ML, Temel $Y$, Ackermans L, Bar-Gad I. Tonic and phasic changes in anteromedial globus Pallidus activity in Tourette syndrome. Movement disorders. 2017.

Bos $M$, Janssen ML, Smeets AY, Temel $Y$, van Zundert A, Ackermans L. Use of dexmedetomidine during deep brain stimulation for Tourette Syndrome: a case report and review of the literature. Anaesthesia cases. 2017.

Hakvoort K, Haeren RH, Smeets AY, van Santbrink H. Extracranial extension of a glioblastoma through a burr hole presenting as a wound healing problem. Tijdschrift voor Neurologie en Neurochirurgie. 2017.

Smeets AY, Duits AA, Verdellen C, de Wert G, Horstkötter D, Temel Y, Ackermans L, Leentjens AF. Ethics of Deep Brain Stimulation in adolescent patients with refractory Tourette Syndrome: a systematic review and two case discussions. Neuroethics. 2018.

\section{CHAPTER IN BOOK}

Smeets AY, Duits A, Leentjens AF, Ackermans L. Chapter Neurosurgical treatment in Tourette Syndrome [Hoofdstuk Neurochirurgische behandeling in Handboek Gilles de la Tourette]. To be published in 2018.

Smeets AY, Leentjens AF, Ackermans L. Chapter Deep Brain Stimulation in Tourette Syndrome. Book: Deep brain stimulation, an interdisciplinary approach. Edited by: Temel Y, Chabardes S, de Bie RMA, Leentjens AFM and Volkmann J. To be published in 2018 by Springer. 


\section{ORAL PRESENTATIONS}

Drop foot correction in post-stroke patients using an implantable dual-channel peroneal nerve stimulator. 26th Symposium Experimenteel Onderzoek Heelkundige Specialismen. Maastricht, the Netherlands, 2013.

Deep Brain Stimulation of the internal globus pallidus in refractory Tourette Syndrome. XXI Congress of the European Society of Stereotactic and Functional Neurosurgery. Maastricht, the Netherlands. 2014.

Thalamic Deep Brain Stimulation for refractory Tourette Syndrome: clinical evidence for increasing disbalance of therapeutic effects and side effects at long-term follow-up. XXII Congress of the European Society of Stereotactic and Functional Neurosurgery. Madrid, Spain. 2016.

Deep Brain Stimulation of the medial thalamus in refractory Tourette Syndrome. Nederlandse Vereniging voor Psychiatrie (NVVP) congres. Maastricht, the Netherlands. 2018.

\section{POSTER PRESENTATIONS}

Smeets AY, Duits AA, Leentjens AF, Schruers K, van Kranen-Mastenbroek V, VisserVandewalle $V$, Temel $Y$, Ackermans L. Long-term follow-up of thalamic Deep Brain Stimulation in refractory Tourette Syndrome. II International Conference on Deep Brain Stimulation. Dusseldorf, Germany. 2016.

Smeets AY, Israelashvili M, Bronfeld M, Zeef DH, Leentjens AF, van Kranen-Mastenbroek V, Janssen ML, Temel Y, Ackermans L, Bar-Gad I. Basal ganglia disinhibition in Tourette Syndrome; patient and animal model of tic expression. XXII Congress of the European Society of Stereotactic and Functional Neurosurgery. Madrid, Spain. 2016.

Smeets AY, Duits AA, Verdellen C, de Wert G, Horstkötter D, Temel Y, Ackermans L, Leentjens AF. Ethics of Deep Brain Stimulation in adolescent patients with refractory Tourette Syndrome. Joint Meeting of the Belgian-Dutch Neurosurgery societies. Den Bosch, the Netherlands. 2017. 
